\title{
Contextual attributes fostering self-regulated learning in a teacher-centered culture
}

Citation for published version (APA):

Matsuyama, Y. (2020). Contextual attributes fostering self-regulated learning in a teacher-centered culture: learners' professional identity formation is a trigger. [Doctoral Thesis, Maastricht University]. Maastricht University. https://doi.org/10.26481/dis.20201005ym

Document status and date:

Published: 01/01/2020

DOI:

10.26481/dis.20201005ym

Document Version:

Publisher's PDF, also known as Version of record

\section{Please check the document version of this publication:}

- A submitted manuscript is the version of the article upon submission and before peer-review. There can be important differences between the submitted version and the official published version of record.

People interested in the research are advised to contact the author for the final version of the publication, or visit the DOI to the publisher's website.

- The final author version and the galley proof are versions of the publication after peer review.

- The final published version features the final layout of the paper including the volume, issue and page numbers.

Link to publication

\footnotetext{
General rights rights.

- You may freely distribute the URL identifying the publication in the public portal. please follow below link for the End User Agreement:

www.umlib.nl/taverne-license

Take down policy

If you believe that this document breaches copyright please contact us at:

repository@maastrichtuniversity.nl

providing details and we will investigate your claim.
}

Copyright and moral rights for the publications made accessible in the public portal are retained by the authors and/or other copyright owners and it is a condition of accessing publications that users recognise and abide by the legal requirements associated with these

- Users may download and print one copy of any publication from the public portal for the purpose of private study or research.

- You may not further distribute the material or use it for any profit-making activity or commercial gain

If the publication is distributed under the terms of Article $25 \mathrm{fa}$ of the Dutch Copyright Act, indicated by the "Taverne" license above, 
Contextual attributes fostering self-regulated learning in a teacher-centered culture: learner's professional identity formation is a trigger. 


\section{Maastricht University 4 Maastricht UMC+}

in the School of Health Professions Education
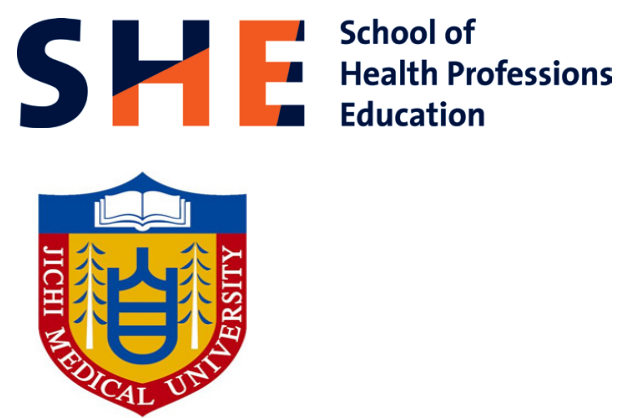

and in Jichi Medical University
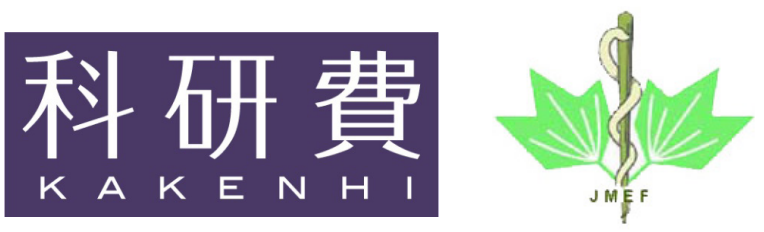

and was funded by JSPS Kakenhi and Japan Medical Education Foundation

ISBN: 978-94-6421-023-1

The copyright of articles that have been published has been transferred to the respective journals. The research reported here was conducted under the supervision of the School of Health Professions Education, Maastricht University, Maastricht, Netherlands,

Copyright (C) Yasushi Matsuyama 


\section{Contextual attributes fostering self-regulated learning in a teacher-centered culture: learner's professional identity formation is a trigger}

\section{DISSERTATION}

To obtain the degree of Doctor at the Maastricht University, on the authority of the Rector Magnificus,

Prof. Dr. Rianne M. Letschert

in accordance with the decision of the Board of Deans,

to be defended in public on

Monday, 5 October 2020 at 10:00 hours

by

YASUSHI MATSUYAMA 


\section{Supervisor}

Prof. Dr. C.P.M. van der Vleuten, Maastricht University, the Netherlands

\section{Co-supervisor}

Dr. Jimmie Leppink, Hull York Medical School, University of York, UK

\section{Assessment committee}

Prof. Dr. A.B.H. de Bruin (Chair)

Dr. J.J. Berkhout (Amsterdam UMC)

Prof. Dr. M.G.A. oude Egbrink

Prof. Dr. M. van der Schaaf (University Medical Center Utrecht)

Dr. S. Schreurs 
For my wife, Rie, and our children Tomoshi, Reiji and Minori: your love, patience and support made this possible. 
Table of contents 
Chapter 2 Contextual attributes promote or hinder self-regulated learning: a qualitative study contrasting rural physicians with undergraduates in Japan.

Published in Medical Teacher 2018; 40(3): 285-295

Chapter 3 Does changing from a teacher-centered to a learner-centered context promote self-regulated learning: a qualitative study in Japanese undergraduate setting. Published in BMC Medical Education 2019; 19: 152

Chapter 4 Education in professional identity formation enhances self-regulated learning: a mixed-method study from a community-based clinical clerkship in Japan.

Under editorial review

Chapter 5 Limited effects from professional identity formation-oriented education on self-regulated learning in a hybrid problem-based learning curriculum: a mixed-method study in Japan

Under editorial review

Chapter 6 Discussion

Summary

Samenvatting

Valorization

Acknowledgments 
Chapter 1

Introduction 


\section{Self-regulated learning as competency in current advances in medicine}

The central theme of this thesis is how to address the challenges of self-regulated learning (SRL) in teacher-centered contexts. Clinical knowledge is rapidly advancing. Medical professionals need to update their knowledge autonomously throughout their professional life. Therefore, selfregulation in learning has come to be an important competency in the lives of medical professionals ${ }^{1,2,3}$.

For more than 30 years, scholars have vigorously examined SRL, and a variety of SRL theories have emerged from different disciplines ${ }^{4}$ (for a review, see Sitzmann and Ely ${ }^{5}$, Zimmerman and Schunk ${ }^{6}$, and Schunk and Greene $\left.{ }^{7}\right)$. However, most theories have four core features, namely 1) a feedback loop: cyclical processes and sub-processes for the purpose of selfmonitoring and strategic learning; 2) motivation: learners' regulation of why and how they choose to self-regulate; 3) goal setting: learners' self-determination of goals, criteria or standards for their academic progress; and 4) self-monitoring or metacognition: the mechanism by which learners keep track of how they are doing or clarify what they are thinking ${ }^{4}$. For instance, Zimmerman's three-phase cyclical model successfully incorporates 2) to 4) in a feedback loop structure and is widely used in a variety of learning contexts, from primary education to vocational training ${ }^{8,9}$. Therefore, this paper also used Zimmerman's three-cyclical model as a theoretical framework. Accordingly, this paper also used his definition of SRL - that is, learners' active participation in their own learning process from metacognitive, motivational, and behavioural perspectives ${ }^{10}$.

Zimmerman's three-phase cyclical model (Figure 1-1) depicts the forethought, performance and self-reflection phases in a cyclical loop form of the learning process ${ }^{3,4,9}$. Each phase has processes and sub-processes that can help decompose and analyze the continuum of individual learning activities, and capture individuals' thoughts, feelings, and actions with regard to the three components of SRL ${ }^{9-11}$. There is an analytic method in line with Zimmerman's three phase cyclical model, so-called "microanalysis" ${ }^{12}$. Essential features of SRL microanalysis include analysis (a) by a single assessor, (b) targeting the phase-specific processes outlined in Zimmerman's loop, (c) with context-specific questions, (d) linking regulatory processes to chronological aspects, and (e) with verbatim recoding and coding of participants' response ${ }^{12}$. In the medical context, Artino et al. asked 71 second-year medical students to work through a paper-based clinical case and investigated the process of their SRL with regard to clinical reasoning by microanalysis, and determined that the novice learners lacked the forethought regulatory process (strategic plans) ${ }^{13}$. Cleary and Sandars investigated the regulatory profiles of students who conducted venipuncture tasks using microanalysis and revealed that students who were successful in venipuncture had high levels of strategic thinking, whereas the students who struggled on this task tended to focus on outcomes ${ }^{14}$. All in all, one style of addressing challenges of SRL in medical education is to approach details of learning processes depending on the learning context. 


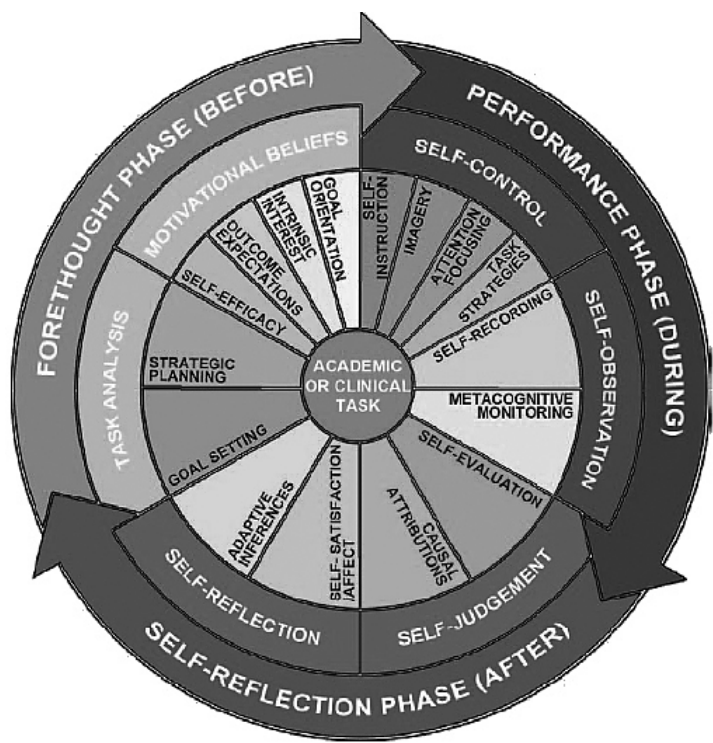

Figure 1-1. Cyclical model of self-regulated learning. Artino AR, Jones KD. Acad Med, 2013, adapted from Zimmerman".

A second approach is to explore contextual attributes that promote SRL in medical education. Several recent studies emphasize that learning context determines whether learners engage in SRL. Brydges and Butler summarized contextual factors influencing SRL ${ }^{15}$ : at the broadest level, from learning expectations from cultural and social communities; and within learning environments, from pedagogical approaches, specific activities and tasks assigned, learning support, and types of feedback or evaluation. Van Houten-Schat et al. specifically shed light on contextual factors influencing SRL in the clinical environment, such as available time, characteristics of learning environment (work climate, team engagement), and patient-related factors ${ }^{16}$. However, most of these studies were conducted in Western cultural contexts. When educational researchers emphasize the importance of contextual attributes in SRL worldwide, greater focus should be placed on different culture context.

\section{Challenges of fostering learners' self-regulated learning in East Asian teacher-centred education}

Education in East Asia is often referred to as Confucian-heritage education. The disciplines originated from the teachings of Confucius, so-called "Confucianism", and these teachings still strongly influence East Asians' values and norms in education, although internationalization and Westernization in recent years have moderated this influence somewhat ${ }^{17}$. 
In Confucian-heritage education, learning is not largely focused on questioning, evaluating, and generating knowledge, because truth is not found primarily in the self. Instead, truth and the good character traits associated with it are primarily learned from the collective; and in particular, from individuals whom the collective recognizes as exemplars, like teachers, or from those ancients whom the collective recognizes as great teachers ${ }^{17,18}$. From primary to secondary education in East Asia, reproducing teachers' statements is strongly emphasized.

One factor which strengthens this propensity is the overemphasis on passing examinations to enter universities. Family members, teachers, and society all ask students in pre-university education to obtain higher grade points and rank in a higher position so that they will be able to attend prestigious universities and assure their future success ${ }^{17}$. Preparatory cram schools also play an important role in submerging students in reproductive learning. Tutors in cram schools devise strategies to repetitively review past lessons (such as past examination papers) and students try to memorize and reproduce what tutors teach in order to prepare for entrance examinations ${ }^{19}$. Entrance examinations emphasize accuracy in the reproduction of informational content, a lowlevel cognitive activity. Therefore, students at matriculation of university are fully accustomed to reproductive learning.

These characteristics remain after they enter universities. University students in an East Asian country, Hong Kong, concentrate their efforts on materials covered by the syllabus and on which they may be examined; they typically rely on handouts or notes taken in lectures, and use texts or recommended readings only as supplements ${ }^{17}$. Students learn to win "the examination game" and expend their energy and time in avoiding failure, which means both personal failure and a disgrace to the family. Likewise, Chinese university students expect their teachers to instruct, and expect themselves to be instructed - "spoon-fed". They rely heavily on handouts and notes taken in lectures ${ }^{17}$. Students expect their teachers to give them explicit directions on what to study, what materials they should read and when to read them. They feel uneasy about unstructured situations or freedom of choice, as manifested for example by asking teachers "What topic should I choose for my assignment?", "What should we discuss today?", and so on.

Self-regulated behaviors are reported to be weaker in East Asian medical universities than in those in the Western world. East Asian students prefer explanatory lectures by teachers to lessen anxiety in PBL discussion, whereas "full" PBL implementation encourages students in the Middle East and the Netherlands to engage in more critical discussions in $\mathrm{PBL}^{20}$. Another report showed that medical students in Japan have difficulty in finding problems prior to self-studying without instructions ${ }^{21}$. In our previous study, we observed consistent reliance on teachers' instruction through lectures and little effort on self-reviewing after a pilot test of progress testing even in Year 5 medical students, who were training in clinical clerkships ${ }^{22}$.

Rich states that the ability to engage in SRL is a key competency that enables residents to learn efficiently through residency program ${ }^{23}$. Sufficient SRL is considered to be a key factor in making learning meaningful in the less-structured educational situation in clinical settings ${ }^{16,24}$. 
Therefore, the lack of readiness for SRL resulting from the current East Asian undergraduate education system is problematic.

The goal of this research project was to identify what delays the development of the three SRL components. To achieve this goal, this research started with a first exploratory study aimed at clarifying how the three major components of SRL function in the context of East Asian medical education: self-metacognition, self-motivation, and behaviors (strategies) of SRL in the current curriculum in East Asia. These findings are considered important for the planned development of a new curriculum designed to promote earlier SRL development after the completion of this project.

In this study, East Asia is defined as "China and the countries that were heavily influenced by its culture, most notably Japan and Korea," in accordance with Nisbett's statement ${ }^{25}$. Because of accessibility to samples and the convenience of qualitative data analysis without obstacles caused by language barriers, we employed Japanese medical students and doctors only. The transferability of findings in this study to East Asian countries can be assured based on their commonality of cultural values ${ }^{16,26}$ and learning strategies in the pre-university phase $e^{17,19}$, the similarity in their traditional undergraduate curricula, and the existence of the national licensing exams as an absolute prerequisite to becoming medical doctors ${ }^{27-29}$.

\section{Problem statement}

The vast increase in expertise and skills that represents the progress of medicine should not be passed on through limited undergraduate education only. Rather, it should be autonomously learned and updated throughout the life of medical professionals $s^{1,2}$. Undergraduate education cannot prepare learners for every challenge arising in less structured learning situations which arise during clinical clerkship and following professional lives ${ }^{16,23,24}$.

Several studies conducted to date have shown that SRL development in East Asian medical students seem insufficient and slow under the current undergraduate education environment $t^{17-22}$. The lack of readiness for SRL resulting from the current East Asian undergraduate education system is problematic. In addition, the causative factors which underlie the delayed development of SRL in East Asian medical students have yet to be comprehensively explored.

To address these issues, the following sections present the research questions asked to address these questions and the outline of this thesis.

\section{Research Questions}

Several overarching research questions arise from the theoretical framework outlined above, which this thesis seeks to address: 
1. What contextual attributes could keep learners in a teacher-centered context from becoming self-regulated learners?

2. What contextual attributes could be triggers for learners in a teacher-centered context to become self-regulated learners?

3. Can we stimulate SRL when introducing education with SRL-triggering attributes?

\section{Thesis Outline}

The study described in Chapter 2 sought to address the first and second research questions by exploring the contrast in self-study between two explicitly different learning contexts in a teacher-centered culture, Japan. We identified a group of rural physicians who were self-regulated learners in a unique postgraduate context, despite having received teacher-centered undergraduate education in Japan. By contrasting their postgraduate and undergraduate context in daily selfstudy, we sought to explore contextual attributes promoting or hampering SRL. Chapter 2 describes a study which uses the grounded-theory method to formulate a theory that explains contextual attributes which promote or hamper SRL in a teacher-centered culture.

Chapter 3 describes a study which uses qualitative methods to explore the effects on SRL when the context changes from teacher-centered to learner-centered, with contextual attributes illuminated by the study in Chapter 2 . This chapter also describes how the contextual change influences SRL. This study concluded that professional identity formation (PIF), or the formation of self-identity via internalization of the values and norms of the profession ${ }^{30}$, influences SRL, and might be a trigger for SRL improvement in teacher-centered cultures.

While these qualitative studies implied that PIF-oriented contextual changes might be important in SRL improvement, they failed to provide a cause-effect relationship between PIForiented context and SRL improvement. Therefore, we created a PIF-oriented education format based on the study findings illustrated in Chapters 2 and 3, and investigated the effects of this PIF-oriented education on SRL in students in both clinical (Chapter 4) and pre-clinical years (Chapter 5).

Chapter 4 describes a mixed-method study which explored the effects of a PIF-oriented education format designed to help undergraduates 1) articulate their future 'self' image as a professional, and 2) have in-depth communication pertaining to medical professionalism with role models in a clinical clerkship. The clinical clerkship setting was a rural community-based clinical clerkship for students of Jichi Medical University (JMU), in which clinical year students study in the future workplace and graduates (role models) directly educate them. This mixedmethod study showed how professional identity induced SRL improvement in clinical-year students.

Chapter 5 describes a mixed-method study which explored the effects of an PIF-oriented education format designed for a preclinical hybrid PBL curriculum in a teacher-centered culture. We created the PIF-oriented pre-PBL learning format to help preclinical year students 1) 
articulate a future 'self'-image as a professional and 2) have in-depth communication pertaining to medical professionalism before every PBL session.

Finally, Chapter 6 contains a discussion of the main findings of the studies in the light of the three main research questions; addresses their strengths and limitations; and considers the implications for medical education and research.

This dissertation is based on separate empirical papers and some overlap will be inevitable.

\section{References}

1. Frank JR. The CanMED 2005 Physician Competency Framework: Better Standards, Better Physicians, Better Care. Ottawa, Canada. Royal College of Physicians and Surgeons of Canada; 2005.

2. World Federation for Medical Education. Continuing Professional Development (CPD) of Medical Doctors: WFME Global Standards for Quality Improvement. University of Copenhagen. 2003.

3. Sandars J, Cleary TJ. Self-regulation theory: application to medical education: AMEE Guide No 58. Med Teach. 2011; 33: 875-86.

4. Artino AR., Brydges R, Gruppen LD. Self-regulated learning in healthcare profession education: theoretical perspectives and research methods. In Researching Medical Education. Edited by: Cleland J, During SJ. Hoboken, NJ: John Wiley \& Sons, Ltd; 2015: 155-66.

5. Sitzmann T, Ely K. A meta-analysis of self-regulated learning in work-related training and educational attainment: what we know and where we need to go. Psychol Bull. 2011; 137: 421-42.

6. Zimmerman BJ, Schunk DH. Self-regulated learning and academic achievement: Theory, research, and practice. NY: Springer Science \& Business Media; 2012.

7. Schunk DH, Greene JA. Historical, contemporary, and future perspectives on self-regulated learning and performance. In Handbook of self-regulation of learning and performance. Edited by; Schunk DH, Greene JA, NY: Routledge; 2018: 1-15.

8. Bembenutty H, Kitsantas A, Cleary TJ. (Eds.). Applications of self-regulated learning across diverse disciplines: A tribute to Barry J. Zimmerman. NC: IAP; 2013.

9. Artino, AR, Jones KD. AM Last Page: Self-regulated learning-a dynamic, cyclical perspective. Acad Med. 2013; 88: 1048.

10. Zimmerman BJ. A social cognitive view of self-regulated academic learning. J Educ Psychol.1989; 81:329-39.

11. Zimmerman BJ. Attaining self-regulation: A social-cognitive perspective. In: Handbook of SelfRegulation. Edited by: Boekaerts M, Pintrich P, Zeidner M. CA: Academic Press; 2000:13-39.

12. Cleary TJ. Emergence of self-regulated learning microanalysis: Historical overview, essential features, and implications for research and practice. In Handbook of Self-Regulation of Learning and Performance. Edited by: Zimmerman BJ, Schunk DH. NY: Routledge; 2011: 329-45.

13. Artino AR., Cleary TJ, Dong T, Hemmer PA, Durning SJ. Exploring clinical reasoning in novices: a self-regulated learning microanalytic assessment approach. Med Educ. 2014; 48: 280-91.

14. Cleary TJ, Sandars J. Assessing self-regulatory processes during clinical skill performance: A pilot study. Med Teach. 2011; 33: e368-74.

15. Brydges R, Butler D. A reflective analysis of medical education research on self-regulation in learning and practice. Med Educ. 2012; 46: 71-9.

16. Van Houten-Schat MA, Berkhout JJ, Van Dijk N, Endedijk MD, Jaarsma ADC, Diemers AD. Self-regulated learning in the clinical context: a systematic review. Med Educ 2018; 52: 1008-15 
17. Ho DYF, Peng S, Chan FS: Authority and learning in Confucian-heritage education: a relational methodological analysis. In Multiple Competencies and Self-Regulated Learning: Implications for Multicultural Education. Edited by: Salili F, Chiu SY. Greenwich, CT, USA: Information Age Publishing; 2001: 29-47.

18. Tweed RG, Lehman DR. Learning considered within a cultural context; Confucian and Socratic approaches. Am Psychol. 2002; 57: 89-99.

19. Kwok P. Examination-oriented knowledge and value transformation in East Asian Cram Schools. Asia Pac Educ Rev. 2004; 5: 64-75.

20. Frambach JM, Driessen EW, Chan LC, Van der Vleuten CPM. Rethinking the globalisation of problem-based learning: How culture challenges self-directed learning. Med Educ. 2012; 46: 738-47.

21. Yoshioka T, Suganuma T, Tang AC, Matsushita S, Manno S, Kozu T. 2005. Facilitation of problem finding among first year medical school students undergoing problem-based learning. Teach Learn Med. 17:136-141.

22. Matsuyama Y, Muijtjens AM, Kikukawa M, Stalmeijer R, Murakami R, Ishikawa S, Okazaki H. A first report of East Asian students' perception of progress testing: a focus group study. BMC Med Educ. 2016; 16: 245.

23. Rich JV. Proposing a model of co-regulated learning for graduate medical education. Acad Med. 2017; 92: 1100-4.

24. Berkhout JJ, Teunissen PW, Helmich E, van Exel J, van der Vleuten CP, Jaarsma DA. Patterns in clinical students' self-regulated learning behavior: a Q-methodology study. Adv Health Sci Educ Theory Pract. 2017; 22: 105-21.

25. Nisbett RE. The geography of thought: How Asians and Westerners Think Differently and Why. NY: Free Press; 2003.

26. Lam TP, Lam YY: Medical education reform: The Asian experience. Acad Med. 2009; 84: 1313-7.

27. Kozu T. Medical education in Japan. Acad Med. 2006; 81: 1069-75.

28. Gojia yīxué kăoshì wăng (National Medical Examination Center): Examination Service. 2013 [http://www.nmec.org.cn/EnglishEdition.html].

29. Kim KJ, Kee C. Reform of medical education in Korea. Med Teach. 2010; 32: 113-7.

30. Cruess RL, Cruess SR, Boudreau JD, Snell L, Steinert Y. Reframing medical education to support the development of a professional identity. Acad Med. 2014; 89: 1446-51. 
Chapter 2

Contextual attributes promote or hinder self-regulated learning: a qualitative study contrasting rural physicians with undergraduate learners in Japan 


\section{Abstract}

Background: Previous studies support the notion that East Asian medical students do not possess sufficient self-regulation for postgraduate clinical training. However, some East Asian physicians who are employed in geographically isolated and educationally underserved rural settings can self-regulate their study during the early phase of their postgraduate career. To explore the contextual attributes that contribute to self-regulated learning (SRL), we examined the differences in self-regulation between learning as an undergraduate and in a rural context in East Asia.

Methods: We conducted interviews and diary data collection among rural physicians $(n=10)$ and undergraduates $(n=11)$ in Japan who undertook self-study of unfamiliar diseases. We analyzed three domains of Zimmerman's definition of SRL: learning behaviors, motivation, and metacognition using constructivist grounded theory.

Results: Rural physicians recognized their identity as unique, and as professionals with a central role of handling diseases in the local community by conducting self-study. They simultaneously found themselves being at risk of providing inappropriate aid if their self-study was insufficient. They developed strategic learning strategies to cope with this high-stakes task. Undergraduates had a fear of being left behind and preferred to remain as one of the crowd with students in the same school year. Accordingly, they copied the methods of other students for self-study and used monotonous and homogeneous strategies.

Conclusions: Different learning contexts do not keep East Asian learners from being selfregulated. Awareness of their unique identity leads them to view learning tasks as high-stakes, and to initiate learning strategies in a self-regulated manner. Teacher-centered education systems cause students to identify themselves as one of the crowd, and tasks as low-stakes, and to accordingly employ non-self-regulated strategies. 


\section{Introduction}

Self-regulated learning (SRL) is defined as learners' active participation in their own learning process from metacognitive, motivational, and behavioral perspectives ${ }^{1}$. In medical practice, clinical knowledge is continuously advancing, and requires extensive and lifelong SRL ${ }^{2.3}$.

In East Asian education, which is often referred to as Confucian-heritage education, virtue is achieved primarily by learning from teachers and imitating their attitudes ${ }^{4.5}$. From primary to secondary education in East Asia or "China and the countries that were heavily influenced by its culture, most notably Japan and Korea", reproducing teachers' statements is strongly emphasized. Family members, teachers, and society urge students in pre-university education to attain higher grade point averages and higher rankings to enable them to attend prestigious universities and assure their future success ${ }^{4}$. Tutors in preparatory cram schools devise strategies to repetitively review past lessons (such as past examination papers) and students try to memorize and reproduce the tutors' teachings to prepare for entrance examinations ${ }^{7}$. Entrance examinations emphasize accuracy in the reproduction of informational content. Therefore, students matriculating at university are fully accustomed to this teacher-centered learning approach.

University students in East Asia expect their teachers to instruct, and themselves to be instructed or "spoon-fed"4. In the context of medical education, one report showed that medical students in Japan have difficulty extracting problems in problem-based learning (PBL) scenarios without instruction from teachers ${ }^{8}$. In addition, medical students in Hong Kong prefer explanatory lectures by teachers to lessen the anxiety of PBL discussions while, in the Middle East and the Netherlands, "full" PBL implementations encourage students to engage in more critical discussions ${ }^{9}$. PBL sessions require students to extract problems and formulate learning goals in a self-regulated manner. In addition, participants should undertake self-study between PBL discussion sessions to accumulate relevant information to make the discussion sessions more meaningful ${ }^{3.10 .11}$. Therefore, the lack of participation by East Asian students may suggest that they are not able to adopt learning strategies that require self-regulation. In our previous study, we found that Japanese medical students consistently relied on teachers' explanatory lectures and had low motivation on self-review after a pilot progress test ${ }^{12}$. This indicates that Japanese medical students are not ready for self-reflection of their study outcomes.

These results support the notion that East Asian medical students rely strongly on teachers' instructions for learning activities, and suggest that undergraduates in East Asia may have fewer opportunities for active participation in SRL. Rich states that the ability to engage in SRL is a key competency that enables residents to learn efficiently through residency programs ${ }^{13}$. Therefore, a lack of readiness for SRL resulting from the current East Asian undergraduate education system is problematic.

Despite this lack readiness, some East Asian physicians can conduct independent selfdisciplined learning without the instruction of teachers in the early stages of their postgraduate careers. For instance, graduates from one medical university in Japan, Jichi Medical University 
(JMU), are employed in a clinic or hospital in medically underserved or geographically isolated areas during postgraduate years (PGY) 3 to 9, where consultation with specialists and educational instruction from teachers is minimal ${ }^{14}$. In this system, JMU graduates work as general practitioners in rural settings for the first few years of their postgraduate careers. The rural settings in which JMU graduates are appointed are geographically isolated and medically underserved, and they accordingly have fewer opportunities to develop their clinical knowledge and skills under systematic instructional programs and have little support from specialists. While many physicians in the early stages of their postgraduate careers prefer to be placed in training hospitals in urban settings, $97 \%$ of JMU graduates complete their contract-based rural services between PGY 3 to 9 in such educationally-underserved settings ${ }^{14}$. In addition, JMU graduates are reported to have a high retention rate in medically underserved prefectures after the end of their contract period and to continue to provide professional care in accordance with rural need $s^{15}$. This indicates that JMU graduates can improve their professional knowledge in educationally underserved settings, and should be sufficient self-regulated learners to remain in such an environment for an extended period of time. This is stark contrast to the fact that the subjects of our previous study ${ }^{12}$, which found that undergraduates were not sufficiently ready to conduct self-study without instruction, were all JMU undergraduates.

This gap between a lack of independent study at the undergraduate stage and lifelong and extensive care provision by self-regulated rural physicians suggests that fundamental attributes, aside from cultural influences, can promote or hinder SRL in learning contexts.

To date, contextual attributes in formally-structured classrooms and clinical environments have been investigated separately. Those in the former setting include social support, feedback, the opportunity for guided and independent practice, support of reflective practice, and the opportunity to make errors; whereas those in the clinical environment include the curriculum, facilities, atmosphere, patient-related factors, available time, the people present, and engagement of the team ${ }^{16.17}$. Recently, Cho et al reported on the changes occurring during the first clinical year among undergraduates according to several categories of the Motivated Strategies for Learning Questionnaire (MSLQ) ${ }^{18}$. The MSLQ is composed of 81 items which quantify the scales of nine types of learning strategies (rehearsal, elaboration, organization, critical thinking, metacognitive self-regulation, time and study environment, effort regulation, peer learning, and help seeking), and six variables of motivation states (intrinsic goal orientation, extrinsic goal orientation, task value, control of learning beliefs, self-efficacy for learning and performance, and test anxiety ${ }^{19}$. However, the types of contextual attributes contributing to these changes have not been specifically investigated.

The aim of this study was to elucidate the contextual attributes promoting or hindering SRL by illuminating differences in learning activities between medical students in teacher-centered undergraduate settings versus rural physicians in geographically isolated and educationally underserved clinical settings in East Asia. 


\section{Methods}

From a constructivist paradigm in which 'reality' is subjective and context-specific, and multiple truths are constructed by and between people ${ }^{20}$, we employed constructivist grounded theory ${ }^{21}$ to elucidate the types of contextual attributes that may promote or hamper SRL in two learning settings. We did not use existing structured measures for SRL, like quantitative scales and SRL microanalysis ${ }^{17}$, which are comprised of open- and close-ended questions that target the forethought, performance and self-reflection phases in a cyclical model of self-regulation. We assumed that these tools did not consider or quantify the characteristics of East Asian learners, and may therefore limit our exploration of these unrecognized characteristics of learners in different cultural contexts. We analyzed differences in self-regulation during learning between two contexts according to Zimmerman's definition of SRL, which comprises metacognitive, motivational, and behavioral perspectives ${ }^{1}$. The questions used in our semi-structured interviews and diary data collection were in line with these three perspectives. We conducted coding and categorizing in combination with theoretical sampling and repetitive comparison according to constructivist grounded theory. For coding, we referred to the terms used for the sub-processes of Zimmerman's three-phase cyclical model ${ }^{22}$. This model depicts three sequential phases of SRL, including forethought (before), performance (during), and self-reflection (after) phases. During the forethought phase, task analysis and motivational beliefs help prepare learners for upcoming learning opportunities. In the performance phase, learners adopt self-control and selfobservation in a metacognitive manner to make progress towards a goal. In the reflection phase, learners employ self-judgement and self-reaction to evaluate performance and guide the next forethought phase for subsequent learning opportunities. We also referred to terms used for learning strategies by the MSLQ ${ }^{19}$, which include rehearsal, elaboration, organization, critical thinking, metacognitive self-regulation, time and study environment, effort regulation, peer learning, and help seeking.

We used physicians working in rural settings, mainly JMU graduates, as representative selfregulated learners. We considered that the high completion rate by JMU graduates of rural medical placement in geographically isolated and educationally underserved settings, and their remarkable medical provision ${ }^{14}$ would be difficult without high levels of SRL. On the other hand, we used JMU undergraduates taught in the standard curriculum as representative nonself-regulated learners because the education board of this university recognizes and is discussing the excessive reliance on teachers and lack of self-regulation by its students (unpublished data). Moreover, our previous study showed that students at JMU across different school years consistently relied on teachers' explanatory lectures and had low motivation on self-review ${ }^{12}$. We conducted theoretical sampling based on the assumption that there is a large difference in learning motivation and behaviors between rural physicians and undergraduates. To illuminate the actual difference, we employed a number of rural physicians and undergraduates of different genders and educational experience. Iterative comparison was conducted by analyzing data from 
one rural physician after one undergraduate, and vice versa, until coding saturation was reached for both groups ${ }^{21.23}$. In the end, 21 subjects (10 rural physicians and 11 undergraduates) were enrolled in our study for one-on-one interviews (Table 2-1). In addition to interviews, we used diaries completed by the same participants to collect a variety of qualitative data ${ }^{21}$. Data collection and analysis were conducted from May 2016 to January 2017.

\section{Subjects}

We primarily employed physicians in rural settings who were graduates from JMU as representatives of self-regulated learners because JMU specifically places general physicians in geographicallyisolated and medically-underserved areas where graduates from other universities are unwilling to work due to the scarcity of training opportunities. All rural physicians invited to participate in this study fulfilled the following inclusion criteria: 1) practiced for more than 2 years in a rural clinic or hospital, where consultation with specialists and regular educational programs were scarce, and 2) currently engaged in primary-to-secondary care in a rural setting. We considered physicians fulfilling these criteria to be sufficiently self-regulated to accomplish learning tasks without the support of specialists. We also employed three doctors (R6, R8, and R9 in Table 2-1) who were graduates of other medical universities in Japan because they had several years of experience in medical practice in a small rural clinic and are widely known as competent rural physicians through the Japanese media or in the Japan Primary Care Association.

We employed undergraduates from JMU to confirm that their learning environment was sufficiently teacher-centered in accordance with our previous study ${ }^{12}$. Undergraduate candidates of different genders, school years, and academic performance (according to test scores) were chosen to ensure variation in these characteristics. Students eligible for a unique student-selected component, the Free Course Student Doctor system, were excluded because we did not consider that this cohort received the traditional lecture- and test-dominated East Asian education: these students were allowed to skip all end-of-unit tests (summative assessment tests) and lectures on clinical medicine in the 6th year in exchange for participation in student-selected clinical clerkships.

\section{Instruments}

\section{1) Interviews}

After informed consent was obtained, a 60-minute semi-structured interview using four questions (see below) was conducted. The main researcher (YM) interviewed each participant and recorded all conversations during the sessions. All recorded interviews were subsequently transcribed by research assistants. 
Table 2-1. Characteristics of interviewees and the unfamiliar diseases discussed.

\begin{tabular}{|c|c|c|c|c|}
\hline No & PGY & Sex & Unfamiliar disease entity & Data source \\
\hline \multirow{2}{*}{$\mathrm{R} 1^{\mathrm{b}}$} & \multirow{2}{*}{13} & \multirow{2}{*}{ M } & External ear carcinoma & Interview \\
\hline & & & Meningitis caused by acute sinusitis & Diary \\
\hline \multirow{2}{*}{$\mathrm{R} 2$} & \multirow{2}{*}{18} & \multirow{2}{*}{$\mathrm{F}$} & Proton pump inhibitor-induced osteoporosis & Interview \\
\hline & & & Chronic hepatitis C & Diary \\
\hline \multirow{2}{*}{ R3 } & \multirow{2}{*}{4} & \multirow{2}{*}{$\mathrm{M}$} & Chronic obstructive pulmonary disease & Interview \\
\hline & & & IgG4-related disease & Diary \\
\hline \multirow{2}{*}{ R4 } & \multirow{2}{*}{6} & \multirow{2}{*}{ M } & Corrected transposition of the great arteries & Interview \\
\hline & & & Obturator hernia & Diary \\
\hline R5 & 16 & M & Non-occlusive mesenteric ischemia & Interview \\
\hline \multirow[b]{2}{*}{ R6 } & \multirow[b]{2}{*}{12} & \multirow[b]{2}{*}{ M } & Stuttering & Interview \\
\hline & & & $\begin{array}{l}\text { Atrial fibrillation during dual antiplatelet } \\
\text { therapy }\end{array}$ & Diary \\
\hline $\mathrm{R} 7$ & 17 & M & Tsutsugamushi disease & Interview \\
\hline R8 & 12 & $\mathrm{M}$ & Multiple system atrophy & Interview \\
\hline R9 & 12 & M & Refractory cancer pain & Interview \\
\hline R10 & 16 & $M$ & Frontotemporal dementia & Interview \\
\hline \multirow{2}{*}{$U^{d} 1$} & \multirow{2}{*}{ Y6 } & \multirow{2}{*}{$\mathrm{M}$} & Dilated cardiomyopathy & Interview \\
\hline & & & Cyclic vomiting syndrome & Diary \\
\hline \multirow{2}{*}{ U2 } & \multirow{2}{*}{ Y6 } & \multirow{2}{*}{$\mathrm{M}$} & Kawasaki's disease & Interview \\
\hline & & & Multiple myeloma & Diary \\
\hline U3 & Y3 & $\mathrm{M}$ & Takayasu's arteritis & Interview \\
\hline \multirow{2}{*}{ U4 } & \multirow{2}{*}{ Y2 } & \multirow{2}{*}{$\mathrm{M}$} & Parkinson's disease & Interview \\
\hline & & & Cushing's syndrome & Diary \\
\hline U5 & Y3 & $\mathrm{M}$ & Basedow's disease & Interview \\
\hline U6 & Y3 & $M$ & Rheumatoid arthritis & Interview \\
\hline U7 & Y6 & $\mathrm{F}$ & Acute appendicitis & Interview \\
\hline U8 & Y4 & $\mathrm{M}$ & Esophageal cancer & Interview \\
\hline U9 & Y3 & $\mathrm{F}$ & Amyotrophic lateral sclerosis & Interview \\
\hline U10 & Y4 & $\mathrm{F}$ & Kawasaki's disease & Interview \\
\hline \multirow{2}{*}{ U11 } & \multirow{2}{*}{ Y2 } & \multirow{2}{*}{ M } & Bacterial enterocolitis & Interview \\
\hline & & & Food poisoning & Diary \\
\hline
\end{tabular}

${ }^{a}$ postgraduate year, ${ }^{b}$ rural physician 1 to $10,{ }^{c}$ school year, ${ }^{d}$ undergraduate 1 to 11 
Because a wide spectrum of learning takes place in medical settings, we specifically examined a learning task in which rural physicians and medical students undertake self-study of a disease that they have newly encountered or recognized through their daily learning activities. We chose this task because learning a disease entity is 1) a fundamental competency for lifelong maintenance of clinical knowledge2, and 2) a common task between medical students and experienced physicians because there are myriads of existing disease entities in medicine, and there are still newly recognized diseases (e.g. IgG4-related diseases) and newly conceptualized diseases (e.g. locomotive syndrome). Questions used in semi-structured interviews, as well as diaries, were formulated to correspond to the three domains comprising the definition of SRL, i.e., 'learning behaviors (strategies)', 'self-motivation', and 'metacognition' ${ }^{24}$.

The first question cued interviewees to recall their recent self-learning experience.

\section{Q1. Can you recollect your latest experience of learning about an unfamiliar disease (from} definition to clinical features, diagnostic procedure, treatment, and prognosis) and describe how you studied this by yourself?

Subsequently, a dialogue was conducted for 45-60 minutes based on the following three questions (Q2 to Q4), which correspond to behaviors (strategies), motivation, and metacognition, respectively:

Q2. In the curriculum or medical practice, how do you usually learn about an unfamiliar disease, and what methods and strategies do you usually apply to self-study?

Q3. Other interests (like watching TV and chatting with friends) can distract from studying. In the curriculum or medical practice, how do you usually motivate yourself, and what methods and strategies do you usually apply for self-motivation?

Q4. In the curriculum or medical practice, how do you usually monitor and assess your understanding about a disease?

2) Diaries

Diaries help participants to develop deeper insights into their perceptions of learning experiences than those captured by reflection of particular instances of learning, and detailed recording provides more details about individual activities ${ }^{25}$. After the interview, each participant was asked to write a diary note composed of the same four questions asked in the interview form. They wrote in the diary when they next conducted self-study of a disease within a few months after the interview. They were requested to write only one diary note because we thought that frequent diary writing might be bothersome. The main researcher (YM) sent a reminder e-mail 
to the participants for diary note submission; however, we had not received diary notes from five rural physicians and seven undergraduates by the time we reached data saturation. We did not follow-up on the reminders to submit diary notes at the time of data saturation because open codes were found to be similar between the interview data and diary data for the same person.

\section{Analysis}

In line with constructivist grounded theory ${ }^{21}$, transcripts from both interviews and diaries were repeatedly analyzed between interview sessions or diary note collection. Open and axial coding and inductive categorization were conducted in Japanese by YM, and codes and categories were thoroughly reviewed by two Japanese researchers ( $\mathrm{HO}$ and $\mathrm{MN}$ ), respectively. The transcripts were analyzed with a focus on statements about learning about unfamiliar diseases. Data collection continued until data saturation, in which no additional codes or categories were identified and the latest interview data were found to be redundant ${ }^{23}$ by the three Japanese researchers (YM, HO, and $\mathrm{MN}$ ). In the selective coding phase, codes and representative speechsupporting codes were translated once into English by professional translators and proof-readers. The other authors (JL and CvdV) subsequently contributed to analysis of the translated data to develop a highly refined version of the generated theory.

\section{Ethical approval and consent to participate}

The study was approved by the university's ethics committee (reference number: 15-154).

\section{Results}

When asked Q1, both rural physicians and undergraduates first recalled the names of unfamiliar diseases that they most recently studied. They simultaneously recollected their work setting; that is, where and when they conducted self-study of the unfamiliar diseases. They described the appearance of the settings and the things within the settings that motivated them to study those diseases. This process helped them to identify a variety of interactions, both physically and emotionally, that occurred between people in the settings and themselves, while they were undertaking self-study. Rural physicians primarily recalled self-study of unfamiliar diseases in the context of their daily practice. The people they recalled were patients diagnosed with an unfamiliar disease, nurses and other medical professionals in the rural areas, and experts outside the rural community. On the other hand, undergraduates, regardless of school year or interview timing, primarily recalled episodes of self-study in the context of preparing for written tests or interview assessments from tutors and faculty members. The people they recalled included classmates, senior 
students and teachers. Both rural physicians and undergraduates eventually identified a locus for acquisition of knowledge where mutual engagement took place between the learner and the surrounding people. Rural physicians tended to view mutual engagement as patient management in their rural communities, whereas undergraduates viewed mutual engagement as surviving in a cooperative manner in the teacher-centered and test-oriented school environment.

Q2 to Q4 inquired about behaviors, motivation, and metacognition, respectively, and helped participants to verbalize the strategies they were employing for these three domains of SRL. When interviewees were asked why they used their described strategies, they primarily explained or justified their behaviors by relating the significance of tasks to the role identified in the locus for acquisition of knowledge.

Therefore, we defined the locus in which interaction between a learner and the surrounding people took place as a learning community. We further defined awareness of one's role in a community as identity formation, evaluation of task significance as task analysis, and strategies based on identity formation and task analysis as coping strategies. It should be noted that there were variations among all participants. However, iterative comparison between physicians in rural settings and undergraduates in the current East Asian curriculum illuminated explicit differences in these aspects, which provided hints towards understanding the different contextual attributes influencing SRL between these groups.

To summarize, rural physicians recollected experiences of self-study for unfamiliar diseases in the learning context where he/she saw his/her identity as unique in the rural community, and considered the learning task as high-stakes. Accordingly, they actively sought learning strategies. Undergraduates recalled self-study of unfamiliar diseases in the learning context of preparing for an exam such as to remain in pace with others under the pressure of tests, and considered his/herself as one of the crowd. Learning tasks were seen as low-stakes to avoid failing tests, and strategies were monotonous and homogeneous (Figure 2-1).

\section{Identity formation}

a) Rural medical setting

Rural physicians perceived themselves to be in a unique position with respect to other members of the community because of their professional knowledge and skills in a geographically isolated and medically underserved environment with few specialists.

'Well, it was more like there was no other choice. There weren't really any other medical institutions around except for mine, you see. Even if I was to refer them to a specialist, I was located a good Go or $70 \mathrm{~km}$ from somewhere like a neurology clinic at the time.' (R10: PGY 16)

'I came to realize that no one would teach me anything if I didn't make an effort on my own when I travelled elsewhere in my third year. It was less like they wouldn't tell me anything and more like they didn't have the knowledge, period. So I pretty much had to do it myself.' (R5: PGY 16) 
Figure 2-1. Contrast of contextual attributes between a rural medical setting and teacher-centered education

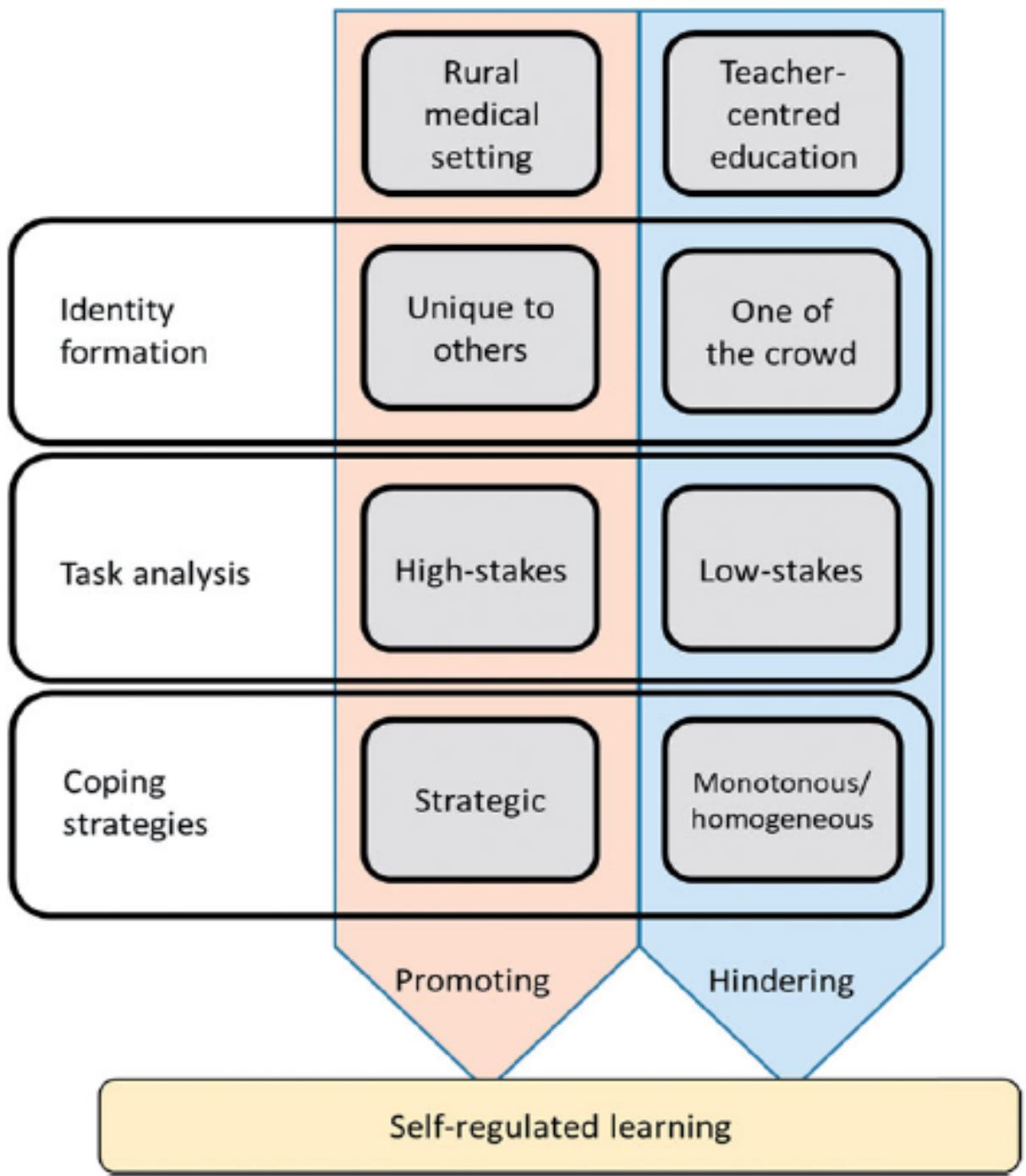

Because of the dearth of alternatives in geographically isolated situations with few specialist resources, the physicians found that they could not rely on the attitude of 'I won't see you, find some other institution' to avoid contextual challenges. Although they were not able to address all medical problems, they tried to find what they could do for patients. 
'I never say "I won't see you, that's it. Find some other institution". Even if I can't treat them myself, I always make sure to work with the patient to guide them on their next move like, "You may be able to get treated if you do this..." and at the same time, I take care to avoid patients on the phone feeling like they were just bluntly refused out of hand.' (R6: PGY 12)

Identity formation as a unique medical professional in the community led to the perception that the physicians had to allow patients to ask them about any wide-ranging medical problem. Rural physicians perceived that they were expected to have broad knowledge as preparation for consultation about any and all medical problems. They described the unique identity of a medical professional in a rural setting as a specialist who allowed patients to consult them about any problem.

'[I feel that] letting patients consult with us about any problem is our specialty.' (R5: PGY 16)

The rural physicians were not stand-alone or self-righteous. They humbly viewed their unique professional abilities as essential and did not think that their sole contribution would improve the practice in the community. Rural physicians recognized that their individual self-study could help to improve the quality of medical practice at the whole community level. They were not confident being the center of the community but perceived themselves as having been invited to the central role, and this perception strengthened their unique identity.

'At first, I just thought of my skills as my own. But recently, I have perceived them as skills for the community...if my skills improve, homecare managers may say that we might do it that way too. If it goes viral, it would result in levelling up of care for entire rural community. But I myself don't have what authentic professionals have, so I am not sure. But I hope the community raises its care levels as a whole.' (Rg: PGY 12)

b) Teacher-centered education

Most undergraduates recalled their test preparation when asked about their latest experience of self-study of unfamiliar diseases. They described that the fear of failing exams outweighed the desire to learn about diseases with clinical significance. They felt that teachers' judgement of their test performance could ruin their individual learning processes.

'Teachers cannot see...the intense effort I make. After all, even though I may understand go\% of the facts of the disease, not knowing the remaining $10 \%$ could result in failure to answer that one question, and if I only know 90\% of the next disease entity, one wrong answer may follow another. A bad test score can result in my being labelled as not studying at all.' (Uı: Year 6) 
Undergraduates also articulated a fear of lagging behind their classmates. They wanted all classmates to pass the exam and graduate in a cooperative manner.

'The urge to graduate along with my current circle offriends is probably the strongest right now, yeah. Studying and learning alongside them in the same class year and being able to graduate with them is what I most want to do.' (U2: Year 6)

From this perspective, they felt comfortable being among other students but felt uncomfortable when there were differences in academic level among the students. As a result, a learning community directed to succeeding in assessments formed among the students. They were likely to view themselves as one of the crowd within the community.

2. Task analysis

a) Rural medical settings

Daily practice and being exposed to a variety of patients' health problems kept them aware of the range of diseases that they needed knowledge of for their unique professional role. The statement "I absolutely have to be prepared to deal with" in the following quote indicates that rural physicians recognized themselves as the only professional in the community who could deal with the variety of diseases.

'What keeps me diligent and never lets my ambition slip is having all kinds of patients visit me every day and knowing that there are a lot of issues I absolutely have to be prepared to deal with.' (R3: PGY 4)

However, their feeling of confidence when addressing the task was not always high. One experienced rural physician described the rural community as a place where he was 'taken hostage'. He noted that rural physicians perceived that they were at risk of making incorrect decisions if they conducted self-study inappropriately, and that all of their mistakes could be scrutinized by the community at any moment. The fear of making mistakes outweighed selfefficacy in their profession in an isolated rural environment.

'I used to treat patients feeling like l'd been taken hostage. Rather than seeing it as my duty, I was focused on what I saw outside my window; rather than seeing this as a step in my life as a physician, I was worried about what people would say about me if I screwed up out here. [...] There was almost nothing that made me happy going to a rural area and meeting sick patients. In fact, what gave me the energy to make it through the day was really the thought of the damage I'd cause if I failed. Like, "I'd better not mess up".' (R7: PGY17)

Such a conflict between the fear of making mistakes and the image of their unique professional role in handling a variety of diseases made them perceive their learning tasks as high-stakes. 
b) Teacher-centered education

Undergraduates had a fear of lagging behind their classmates and preferred to be one of the crowd in their learning communities, where they were driven to learn about unfamiliar diseases so as not to fail exams. They monitored their understanding about unfamiliar diseases through conversations with other students in the same school year. If they recognized that other members had a better understanding of diseases than themselves, they attempted to decrease the difference. However, if they felt themselves able to keep up, they tended to be satisfied regardless of their depth of understanding of the disease.

'I feel like l'm able to more or less keep up with the others in my group when talking on the way to our department. I realize I'm simply comparing myself to others, but feeling like I'm not lagging behind is one of the basic criteria'. (U5: Year 3$)$

Undergraduates did not base their learning goals on their own absolute understanding or clinical significance when they learned about unfamiliar diseases. They were likely to reconcile outcomes of self-study to those of other members. For them, the aim of learning about unfamiliar diseases was not associated with acquiring applicable knowledge for their future medical practice.

'Well, I'm not terribly bright, so my test scores have never been phenomenal. But I think there's a bit of a difference between being able to ace a test and what you're capable of doing once you're a doctor. Studying for a test differs from studying for my future for what will be applicable later.' (U3: Year 3)

Therefore, they were not fully encouraged to deepen their understanding about unfamiliar diseases. Overall, students' perceptions about the irrelevance of test outcomes for clinical competency, and minimum goal setting based on the understanding of other community members could cause undergraduates to perceive learning tasks as low-stakes.

3. Coping strategies

a) Rural medical settings

To cope with what they viewed as high-stakes tasks, rural physicians began to employ some learning strategies noted in Zimmerman's model and Pintrich's MSLQ, such as attention focusing, self-reflection, and critical thinking. For example, they strengthened "attention focusing" by articulating the aim of the self-learning to clarify their learning goals. As they needed to provide concrete solutions for numerous clinical problems, they chose to focus on specific domains like diagnosis, treatment, and prognosis of current clinical problems, rather than developing a superficial understanding of the whole domain of the new disease. 
'For a given patient, I was first concerned with how to proceed with the initial examination - like how should I make my diagnosis, how should I set things up. I would then look into the treatment once we reached the treatment stage later on.' (R1: PGY 14)

Physicians became highly attentive to small errors in their understanding because they perceived that inappropriate knowledge could cause negative outcomes. For example, when providing information on diseases to patients, rural physicians were likely to evaluate the accuracy of their own understanding in a very attentive manner.

'I worried that something I said might kill the patient. That fear dogs me even now.' (R7: PGY17)

Focused attention to detailed knowledge about unfamiliar diseases encouraged rural physicians to base their understanding on reliable information. They rigorously sought information about unfamiliar diseases from as many resources as possible, and compared the quality of information among resources. They attempted to access information authorized by specialist committees (including guidelines), comments from experienced experts, and online information sources updated on a regular basis (e.g. Uptodate ${ }^{\oplus}$ ), and simultaneously critically evaluated the quality of the information ("critical thinking" in MSLQ).

'Eager to obtain basic information about an obturator hernia as quickly as possible, I planned to read UptoDate's summary and recommendation sections. However, when I searched UptoDate, I only found the term "obturator hernia" with no subsections offocus, and I eventually gave up when all I could find was a large summary on intestinal obstruction. The situation was the same when I consulted Harrison, which lacked even the basic information I wanted to know. [...] I checked a textbook I found on the desk of my colleague, but it was no help. [...] For the time being, I searched for articles using Google, PubMed, and Medical Online.' (R4: PGY 6)

They began to evaluate the accuracy of acquired knowledge in a metacognitive manner by asking for diverse opinions outside the rural community. Some rural physicians intentionally presented their knowledge via social networking sites after learning about unfamiliar diseases to receive feedback for self-reflection.

'I have my own internet site and regularly post what I study...I conduct self-evaluations and receive feedback. In the process, I perform self-reflection, which may be a way of learning about myself.' (R7: PGY17)

b) Teacher-centered education

Their learning goals were associated with passing an exam. The range of information that they acquired about new diseases was likely to correspond to the range of subjects studied for test preparation. 
'I sometimes skip parts of learning materials that were not tested in the past.' (U3: Year 3)

Undergraduates limited their learning to the extent of other students' knowledge of unfamiliar diseases rather than the disease's clinical significance. When they found learning materials difficult to understand, they sought answers from agreement among peers (mainly like-minded friends and sometimes senior students); however, they avoided critical thinking and did not attempt to find more reliable and authentic information by referring to a variety of information sources or consulting experts.

'When the word is still unclear after looking it up again and again, well, I will ask my friend who is good at studies for a general outline of the word, and pretend to get it.' (U7: Year 6)

They did not attempt to study more detailed learning contents than their classmates. They preferred to use materials like resumes (summary of lectures) prepared by teachers and textbooks recommended from other students because the provided information was considered to be highly likely to be tested.

'What is written in resumes may be what is asked in tests. And textbooks are so thick that I would wonder what is important... of course all of it important, but I can learn what is really important by referring to the domains written in resumes.' (U11: Year 2)

They were eventually likely to undertake self-study in a monotonous and homogeneous manner.

\section{Discussion}

This study revealed the differences in Zimmerman's three domains of SRL (motivation, behaviors, and metacognition) when engaging in the same learning task (self-study about unfamiliar diseases) in two distinct learning contexts (rural physicians and undergraduate medical students). Rural physicians undertook self-study of unfamiliar diseases in a highlymotivated, strategic, and metacognitive manner according to their unique identity in a rural community. Undergraduates in East Asia conducted self-study in a monotonous and homogeneous manner that was dependent on the behaviors of their classmates and not on themselves in a community engaged in test preparation. Although the rural physicians and undergraduates in this study received the same conventional undergraduate education, referred to as Confucian-heritage education, the two groups of learners employed different levels of self-regulation in motivation, behaviors and metacognitions. This indicates that contextual attributes can influence SRL differently even when learning tasks and previous learning experiences are the same. 
Rural physicians mainly recalled how they perceived and what strategies they used in selfstudy of an unfamiliar disease in their daily practice. In contrast, most undergraduates, regardless of school year and timing of interview, recalled episodes of self-study in the context of preparing for exams. Although there are many other learning situations for undergraduates and rural physicians, we think that our methods effectively illuminated two distinct learning contexts, both of which were relevant to and are representative of daily learning activities for both groups.

The questions used in the interviews and diaries focused on self-study activities in a particular self-study setting. This could create a contrast between self-activity and the surrounding environment, and might help participants articulate the activities of individual learners and existing factors, including other members in the environment. Wenger claims that learning for individuals is not only an internal process but rather an issue of engaging in and contributing to the practices of communities ${ }^{26}$. In his definition, communities are not just spatial structures like a residential neighborhood. He coined the phrase "communities of practice", which he defines as "groups of people who share a concern, set of problems, or passion about a topic, and who deepen their knowledge and expertise in this area by interacting on an ongoing basis ${ }^{27}$." He associated learning with the community of practice in his book ${ }^{26}$. He claims that practices evolve as histories of learning are shared through participation and reification in communities, and emphasizes that access to competence and personal experience of mutual engagement in learning enhances identity formation in learning communities. In other words, learning communities are a "place of identity to the extent they make trajectories possible ${ }^{26}$."

In this study, we observed two communities of practice. The first involved experienced nurses, other medical professionals, homecare managers, physicians with unique professional knowledge and skills, and locals who continuously scrutinize new rural physicians. Although in reality rural physicians learned unfamiliar diseases alone, qualitative data indicated that their perceptions and actual behaviors were based on the learning community as a community of practice, and was aimed at patient management in the rural area. Rural physicians felt that the community gave them full responsibility for the outcomes of their learning tasks, because there was no substitute expert medical practitioner in the community. In line with the theory of community of practice ${ }^{26}$, rural physicians are highly inclined to experience insider trajectories that prompt them to evolve the community through high accountability. A quote from a rural physician saying his self-study can "result in levelling up of care for entire rural community" might support this idea. In other words, rural physicians perceive that their learning activities, being based on their unique identities in the community, contribute to the improvement of patient management in their rural community.

In contrast, undergraduates formed a community of practice in which mutual engagement was "not to fail assessment tests nor be left behind others." Undergraduates did not actively evolve the community by participating in the central role or intentionally place themselves outside the community for fear of isolation. The theory of community of practice indicates that identity formation takes place by identification and negotiability through participation or non- 
participation in certain communities ${ }^{26}$. Therefore, by positioning themselves between the center and the periphery of the community, and by simply doing as others do without active negotiation, the identities of undergraduates became less explicit or more "one of the crowd".

Their preference for being "one of the crowd" and their monotonous and homogeneous learning behaviors might be interpreted as collectivism, which is often characterized as being unique to East Asian cultures. Hofstede identified important elements that account for differences in behaviors between cultures: individualism and collectivism ${ }^{28}$. In collectivistic cultures, members are concerned with the well-being of the in-group, and tend to prioritize the goals of the in-group. They control their behaviors such as remain in line with the in-group's norms rather than for personal purposes. Members of individualistic cultures are likely to focus on personal goals over those of the society. East Asia is often used as a representative of a collectivistic culture, while Western cultures, such as the United States, are used as an example of an individualistic culture ${ }^{28}$. However, we want to emphasize that, even if collectivistic learning values prevail in East Asia, contextual attributes in a different learning setting do not keep East Asian learners collectivistic and passive; rather, contextual attributes encourage them to become independently self-regulated.

It is possible that rural physicians, who are placed in an isolated environment, would accordingly simply be obliged to study alone, and these actions may therefore not be associated with SRL. However, SRL is different to simple self-learning. SRL involves seeking help from experts and various resources to deepen understanding, even when learners are situated in isolated environments ${ }^{29.30}$. In this study, rural physicians were eager to seek authorized information using online resources (UptoDate ${ }^{\circ}$ ) and feedback from experts and colleagues not only with in the community but also outside the community via social networking sites. They did not allow their isolated situation to compromise them or behave in a dogmatic manner.

It could also be viewed that rural physicians were obliged to conduct self-study merely for fear of "making mistakes" and "being scrutinized by the community", indicating that their motivation is external, which is contradictory to intrinsic motivation emphasized by SRL ${ }^{31}$. However, rural physicians attempted to expand the contents of their learning as much as possible, and extended the domain of knowledge in response to practical needs. Rural physicians did not compromise their learning by pursuing it only so far as to avoid criticism from the rural community, in contrast to undergraduates, who pursued learning merely so far as to avoid failing tests. Their state of motivation could be explained by the self-determination theory proposed by Deci and Ryan ${ }^{32.33}$. According to this theory, there are four stages of regulation within extrinsic motivation between amotivation and intrinsic motivation. Learners can proceed through the four stages of external, introjected, identified, and integrated regulation as their self-regulation shifts from controlled to autonomous. The integration of regulation, in which learners can connect extrinsic rules to their own norms and values, is the closest stage to intrinsic regulation (or motivation). Rural physicians in this study were able to internalize the pressures arising from their fear of "making mistakes" and "being 
scrutinized by the community" and accept these perceptions by combining them with their unique but socially important identity.

Overall, this qualitative study shows that immersion in a responsible individual role, which promotes identity formation in the learning community, causes learners to view learning tasks as high-stakes, and to initiate learning strategies in a self-regulated manner. Contextual attributes can promote SRL even among East Asian populations, who are believed to lack SRL during undergraduate education. On this basis, insufficient SRL should not be attributed to the cultural values of learners, but rather to contextual attributes existing in the current teacher-centered undergraduate curriculum. The contextual attributes hindering SRL might include feeling the need to be "one of the crowd" or decreasing individual identity formation, which might cause learners to view learning tasks as low-stakes and employ learning strategies in a monotonous and homogeneous manner.

Application of our results to the current undergraduate education system for further development of SRL may help to form a more identity-oriented learning context. Learning contexts can be individualized in accordance with learners' abilities and tasks can be imposed with sufficient responsibility.

\section{Strengths and limitations}

The strength of this study is that we illuminated the contextual attributes that promote or hinder SRL by contrasting learning activities between learners in two different contexts within the same culture. By contrasting the two contexts in the same culture, we were able to show that fundamental contextual attributes which influence SRL are independent of cultural characteristics. As a result, these findings are expected to be generalizable to other cultural contexts.

One limitation of this study is that we compared self-study activities between two groups in different developmental phases. Rural physicians, who have had longer careers than undergraduates, could have utilized their prior knowledge and experience to behave in a more self-regulated manner. Therefore, the self-regulated characteristics of rural physicians could be attributed to their maturity as professional doctors rather than to any rural context. We could have compared the self-study activities of physicians of the same PGY in rural settings and nonrural settings. However, we used undergraduates to confirm that their learning environment was sufficiently teacher-centered, in accordance with our previous study ${ }^{12}$.

Another limitation of this study is that we mainly investigated undergraduates at a single medical university in Japan. JMU is well known for its high passing rate in the national licensing exam, so undergraduates might be more motivated to follow suit in their learning activities. This study, however, showed that rural physicians who received the same conventional undergraduate education (JMU undergraduate curriculum) could become self-regulated after being employed 
in rural settings. From this point of view, limiting the subjects to one university may have helped to illuminate the difference between 'before' and 'after' exposure to a learning context, which requires full participation as a unique role in a learning community.

Finally, this study only investigated learning activities of self-study with regard to knowledge of unfamiliar diseases. Learners' competencies for self-regulation in learning are applied not only to self-study aimed at clinical knowledge but also learning in groups or of clinical skills. Recent theories suggest that self-regulation in learning can be developed through social transactions, which is considered the central core of regulated learning ${ }^{13}$. In the context of this study, rural physicians might develop competency for self-regulation through interactions with experienced nurses in a flattened hierarchy during staff meetings, whereas undergraduates might avoid seeking help because of scarce interactions with teachers who are higher up in the hierarchy. This study only explored individual learners' perceptions and past experiences according to the conventional social cognitive model of SRL. Future studies should focus on dynamic social interactions influencing SRL.

\section{Conclusion}

A learning context with immersion in a socially responsible role promotes physicians' identity formation as a unique professional. This may cause them to view learning tasks as high-stakes, and lead to the initiation of SRL strategies. In contrast, teacher-centered learning environments may cause students to identify themselves as one of the crowd, and to view tasks as low-stakes. Accordingly, they employed monotonous and homogeneous strategies.

\section{Practice points}

- Contextual attributes can promote SRL among East Asian learners, who are believed to lack the readiness for SRL in teacher-centered undergraduate education cultures. The quality of SRL should be ascribed to learning contexts rather than local cultural characteristics.

- Immersion in a unique professional role promotes awareness of a novel identity, which motivates individuals to view learning tasks as high-stakes and to initiate learning strategies in a self-regulated manner.

- Teacher-centered education causes students to identify themselves as one of the crowd and tasks as low-stakes. They accordingly employ non-self-regulated strategies.

- To invoke self-regulated learning, learning contexts should be more identity-oriented, and learning tasks should be imposed such that learners hold responsibility for their individual roles. 


\section{References}

1. Zimmerman BJ. A social cognitive view of self-regulated academic learning. J Educ Psychol. 1989; 81:329-39.

2. Frank JR. The CanMED 2005 Physician Competency Framework: Better Standards, Better Physicians, Better Care. Ottawa, Canada: Royal College of Physicians and Surgeons of Canada; 2005.

3. Sandars J, Cleary TJ. Self-regulation theory: application to medical education: AMEE Guide No 58. Med Teach. 2011; 33: 875-86.

4. Ho DYF, Peng S, Chan FS: Authority and learning in Confucian-heritage education: a relational methodological analysis. In Multiple Competencies and Self-Regulated Learning: Implications for Multicultural Education. Edited by: Salili F, Chiu SY. Greenwich, CT, USA: Information Age Publishing; 2001: 29-47.

5. Tweed RG, Lehman DR. Learning considered within a cultural context; Confucian and Socratic approaches. Am Psychol. 2002; 57: 89-99.

6. Nisbett RE. The geography of thought: How Asians and Westerners Think Differently and Why. New York (NY): Free Press; 2003.

7. Kwok P. Examination-oriented knowledge and value transformation in East Asian Cram Schools. Asia Pac Educ Rev. 2004; 5: 64-75.

8. Yoshioka T, Suganuma T, Tang AC, Matsushita S, Manno S, Kozu T. Facilitation of problem finding among first year medical school students undergoing problem-based learning. Teach Learn Med. 2005; 17: 136-41.

9. Frambach JM, Driessen EW, Chan LC, Van der Vleuten CPM. Rethinking the globalisation of problem-based learning: How culture challenges self-directed learning. Med Educ. 2012; 46: 738-47.

10. Loyens SM, Magda J, Rikers RM. Self-directed learning in problem-based learning and its relationships with self-regulated learning. Educ Psychol Rev. 2008; 20: 411-27.

11. Sungur S, Tekkaya C. Effects of problem-based learning and traditional instruction on selfregulated learning. J Educational Res. 2006; 99: 307-20.

12. Matsuyama Y, Muijtjens AM, Kikukawa M, Stalmeijer R, Murakami R, Ishikawa S, Okazaki H. A first report of East Asian students' perception of progress testing: a focus group study. BMC Med Educ. 2016; 16: 245.

13. Rich JV. Proposing a model of co-regulated learning for graduate medical education. Acad Med. 2017; 92: 1100-4.

14. Matsumoto M, Kajii E. Medical education program with obligatory rural service: analysis of factors associated with obligation compliance. Health Policy. 2009; 90: 125-132.

15. Matsumoto M, Inoue K, Kajii E. Long-term effect of the home prefecture recruiting scheme of Jichi Medical University, Japan. Rural Remote Health. 2008; 8: 930.

16. Berkhout JJ, Helmich E, Teunissen PW, Berg JW, Vleuten CP, Jaarsma ADC. Exploring the factors influencing clinical students' self-regulated learning. Med Educ. 2015; 49: 589-600.

17. Sitzmann T, Ely K. A meta-analysis of self-regulated learning in work-related training and educational attainment: what we know and where we need to go. Psychol Bull. 2011; 137: 421-42.

18. Cho KK, Marjadi B, Langendyk V, Hu W. Medical student changes in self-regulated learning during the transition to the clinical environment. BMC Med Educ. 2017; 17: 59.

19. Pintrich PR, Smith D, Garcia T, McKeachie WJ. A manual for the use of the Motivated Strategies for Learning Questionnaire (Technical Report 91-B-004). Michigan, USA: The Regents of the University of Michigan; 1991. 
20. Bergman E, de Feijter J, Frambach J, Godefrooij M, Slootweg I, Stalmeijer R, van der Zwet J. AM last page: A guide to research paradigms relevant to medical education. Acad Med. 2012; 87: 545.

21. Creswell JW. The process of conducting research using quantitative and qualitative approaches. In Educational Research. 4th ed. Edited by: Creswell JW. Boston, USA: Pearson; 2012: 15-70

22. Artino AR, Jones KD. AM Last Page: Self-regulated learning-a dynamic, cyclical perspective. Acad Med. 2013; 88: 1048.

23. Hennink MM, Kaiser BN, Marconi VC. Code saturation versus meaning saturation: How many interviews are enough? Qual Health Res. 2016; 27: 591-608.

24. Zimmerman BJ, Pons MM. Development of a structured interview for assessing student use of self-regulated learning strategies. Am Educ Res J. 1986; 23: 614-28.

25. Porto M. Learning diaries in the English as a foreign language classroom: A tool for accessing learners' perceptions of lessons and developing learner autonomy and reflection. Foreign Lang Ann. 2007; 40: 672-96.

26. Wenger E. Communities of practice: Learning, meaning, and identity. Cambridge (UK): Cambridge university press; 1998.

27. Wenger E, McDermott RA, Snyder W. Cultivating communities of practice: A guide to managing knowledge. Massachusetts: Harvard Business Press; 2002.

28. Hofstede G. Culture's Consequences: comparing values, behaviours, institutions, and organizations across nations. Thousand Oaks: Sage Publications, Inc; 2001.

29. Ryan AM, Pintrich PR. 1997. "Should I ask for help?" The role of motivation and attitudes in adolescents' help seeking in math class. J Educ Psychol. 1997; 89: 329.

30. Newman RS. The motivational role of adaptive help seeking in self-regulated learning. In Motivation and self-regulated learning: Theory, research, and applications. Edited by: Schunk DH, Zimmerman BJ. New York (NY): Routledge; 2012: 315-37.

31. Reeve J, Ryan R, Deci EL, Jang H. Understanding and promoting autonomous self-regulation: A self-determination theory perspective. In: Motivation and self-regulated learning: Theory, research, and applications. Edited by: Schunk DH, Zimmerman BJ. New York (NY): Routledge; 2012: 223-44.

32. Ryan RM, Deci EL. Self-determination theory and the facilitation of intrinsic motivation, social development, and well-being. Am Psychol. 2000; 55: 68-78.

33. ten Cate OTJ, Kusurkar RA, Williams GC. How self-determination theory can assist our understanding of the teaching and learning processes in medical education. AMEE guide No. 59. Med Teach. 2011; 33: 961-73. 
Chapter 3

Does changing from a teacher-centered to a learner-centered context promote self-regulated learning: a qualitative study in a Japanese undergraduate setting 


\section{Abstract}

Background: Previous studies indicate that a teacher-centered context could hinder undergraduates from self-regulated learning (SRL), whereas a learner-centered context could promote SRL. However, SRL development between a teacher-centered and a learner-centered context has not directly compared in undergraduate settings. Also, it is still unclear how a contextual change toward learner-centered learning could influence SRL in students, who are strongly accustomed to teacher-centered learning.

Methods: We conducted three focus groups that examined 13 Japanese medical students who left a traditional curriculum composed of didactic lectures and frequent summative tests and entered a seven-month elective course (Free Course Student Doctor or FCSD). The FCSD emphasizes student-designed individualized learning with support and formative feedback from mentors chosen by students' preference. We also conducted two focus groups that examined 7 students who remained in the teacher-centered curriculum during the same period. Students were asked to discuss their 1) motivation, 2) learning strategies, and 3) self-reflection on selfstudy before and during the period. Data were analyzed using thematic analysis and code comparison between the two cohorts.

Results: The non-FCSD participants described their motivational status as being one among a crowd set by the teacher's yardstick. Their reflection focused on minimizing the gap between themselves and the teacher-set yardstick with strategies considered monotonous and homogeneous (e.g. memorization). FCSD participants described losing the teacher-set yardstick and constructing their future self-image as an alternative yardstick. They compared gaps between their present status and future self-image by self-reflection. To fill these gaps, they actively employed learning strategies used by doctors or mentors, leading to diversification of their learning strategies.

Conclusions: A contextual change toward learner-centered learning could promote SRL even in students strongly accustomed to teacher-centered learning. In the learner-centered context, students began to construct their self-image, conduct self-reflection, and seek diverse learning strategies by referring to future 'self' models.

Keywords: Self-regulated learning, Teacher-centered learning, Learner-centered learning, Curriculum reform, Undergraduate education 


\section{Background}

Because clinical knowledge is rapidly advancing, doctors are expected to self-regulate their learning and update their knowledge autonomously in less structured learning settings in medical practice ${ }^{1-4}$.

Self-regulated learning (SRL) is defined as learners' active participation in their own learning process from metacognitive, motivational, and behavioral perspectives ${ }^{5}$. SRL has been theorized as an orderly, cyclical (meta)cognitive process. For instance, Zimmerman described SRL as a cyclical process composed of three phases. In the forethought phase, learners set learning goals and choose a strategy for attaining goals. In the performance phase, learners monitor and control their behavior to attain goals. In the self-reflection phase, learners formulate new learning goals and strategies for similar situations in future ${ }^{6,7}$.

Now, SRL is considered a key competence for medical students, because residency training cannot prepare residents for every challenge their qualification brings ${ }^{8}$. Furthermore, SRL obtained during undergraduate education could lead to life-long learning ${ }^{4}$. Therefore, lack of readiness to engage in SRL resulting from the undergraduate education system is problematic.

\section{Contextual factors influencing SRL}

Several recent studies emphasize learning context determines whether learners engage in SRL. Brydges and Butler ${ }^{3}$ summarized contextual factors influencing SRL: At the broadest level, from learning expectations from cultural and social communities; within learning environments, from pedagogical approaches, specific activities and tasks assigned, learning support, and types of feedback or evaluation. Van Houten-Schat et al. ${ }^{9}$ specifically shed light on contextual factors influencing SRL in the clinical environment, such as available time, characteristics of learning environment (work climate, engagement in team), and patient-related factors.

In a study comparing SRL in self-study between undergraduates in a teacher-centered curriculum and physicians in a rural clinical setting, Matsuyama et al. ${ }^{10}$ identified contextual factors that may hinder SRL in a teacher-centered curriculum. They included students' preference to stay close to fellow students, and engaging in monotonous and homogeneous strategies to avoid failing teachers' assessments. However, postgraduate rural contexts did not keep those learners from being self-regulated. They achieved self-regulation in self-study via 1) awareness of their own unique identity in the learning community, 2) high-stakes tasks which require full responsibility for learning, and 3) experience of coping strategies to complete these high-stakes tasks. Another article reports possible negative effects of teacher-centered undergraduate curriculum on SRL ${ }^{11}$. This demonstrates decrease in cognitive strategic use and self-regulation and increase in anxiety over teacher-centered lectures and summative tests over time.

Moreover, one recent article reveals possible effects of a shift toward a learner-centered 
context on SRL. It shows the introduction of individualized learning plans with support of mentors during four-week clinical clerkship improved self-efficacy and self-regulation among undergraduates ${ }^{12}$. Taking these results into consideration, to foster SRL in undergraduates in preparation for postgraduate training, a learner-centered context might be more beneficial than teacher-centered. However, there is no investigation directly comparing effects on SRL between a learner-centered context and a teacher-centered context in undergraduate settings.

\section{Challenges when changing to a learner-centered context in a teacher-centered culture}

Medical curriculum reforms from teacher-centered learning to learner-centered learning are proceeding worldwide, based on evidence and theories established mainly in the Western world ${ }^{13}$. In the midst of reformation, contextual changes from teacher-centered to learnercentered learning could challenge students, who are strongly accustomed to teacher-centered education culture $\mathrm{e}^{14-17}$.

For example, teacher-centered education culture is still reported in East Asia or "China and the countries that were heavily influenced by its culture, most notably Japan and Korea"18. Traditionally, East Asian education culture is often referred to as Confucian-heritage education where virtue is achieved primarily by learning from teachers and imitating their attitudes ${ }^{19,20}$. Even today, there is still a notable emphasis in primary and secondary East Asian education on reproducing teachers and textbook information. Moreover, in pre-university education, students are urged to attain higher grade point averages and rankings to enable them to attend prestigious universities for future success ${ }^{19}$. Overall, entrance examinations for universities emphasize accuracy in the reproduction of informational content. Tutors in preparatory cram schools devise strategies to repetitively review past lessons (such as past examination papers) to prepare for entrance examinations ${ }^{21}$. This pedagogy may cause East Asian medical students to be fully accustomed to teacher-centered education when entering universities.

This entails challenges when these medical students encounter curriculum reforms from a teacher-centered to a learner-centered context. Yoshioka et al. ${ }^{14}$ report that Japanese medical students have difficulty extracting problems without instruction from teachers in problem-based learning (PBL) in a learner-centered context. Frambach et al. ${ }^{15}$ report that medical students in Hong Kong had anxiety about PBL discussions and asked for explanatory lectures from teachers. As the introduction of learner-centered philosophy challenges learners in teacher-centered cultures in various parts of the world ${ }^{16,17}$, educators can explore how a contextual change toward learner-centered learning could influence SRL in students, who are strongly accustomed to teacher-centered learning, as a general issue. 


\section{Present study}

The aim of this study was to explore whether contextual changes from a teacher-centered to a learner-centered learning could improve SRL in an undergraduate setting. To clarify the aim of this study, we formulated two related research questions: 1) Does change from a teacher-centered to a learner-centered context stimulate SRL; and 2) how does SRL develop during transition from a teacher-centered to a learner-centered context. To address these research questions, we compared self-regulation in learning between medical students who were experiencing contextual change from teacher-centered to learner-centered learning and those who remained in teacher-centered curriculum at the same school year period.

The study was approved by Jichi Medical University Clinical Research Ethics Committee (reference number: 15-154). Informed consent was obtained from all participants. Data collection was conducted from July 2017 to January 2018. Data analysis was conducted in parallel with data collection from November 2017 to March 2018.

\section{Methods}

\section{Settings}

\section{Current Jichi Medical University curriculum as teacher-centered learning context}

Jichi Medical University (JMU) in Japan is a publicly funded medical school whose mission is to increase the number of rural doctors and employ them nationwide. In the current curriculum at JMU (Table 3-1), students finish lectures on almost every subject in basic and clinical medicine before end of Year 3. From Year 4 to May in Year 6, students are permitted to participate in a clinical clerkship, during which they receive training centered mainly on taking patient histories and providing physical examination, but teachers prefer to provide relevant information via lectures rather than the medical practice. Even though they are in the clinical clerkship, they are mainly assessed by an annual comprehensive summative test (Year 4 and 5 Sougouhantei Shiken), which require them to recall knowledge conveyed by teachers. Moreover, Year 6 students must receive didactic lectures on 17 clinical subjects, and take and pass summative tests for each clinical subject. JMU has a good reputation for its high pass rate in exams ${ }^{22}$. However, a previous study ${ }^{10}$ revealed that medical students at JMU perceived the current curriculum as teachercentered and test-oriented, and teacher judgements based on their test performance neglected their individual learning processes.

\section{A novel student-designed elective course as learner-centered context}

In 2011, the Education Board at JMU designed a novel program named the Free Course Student Doctor (FCSD). Students, whose scores on the annual summative test at Year 5 are 
higher than the average scores of Year 6 students, are considered knowledgeable enough to pass all summative tests in Year 6 and the national licensing exam to qualify them as doctors. For these advanced students, the Board has established an elective course with individualized learning plans with support and formative feedback from mentors. For 7 months, the FCSD allows students to study any subject they like at any institution throughout the world and are exempt from all didactic lectures and summative tests for 17 clinical subjects (Table 3-1).

Table 3-1. Undergraduate curriculum at Jichi Medical University and the Free Course Student Doctor period

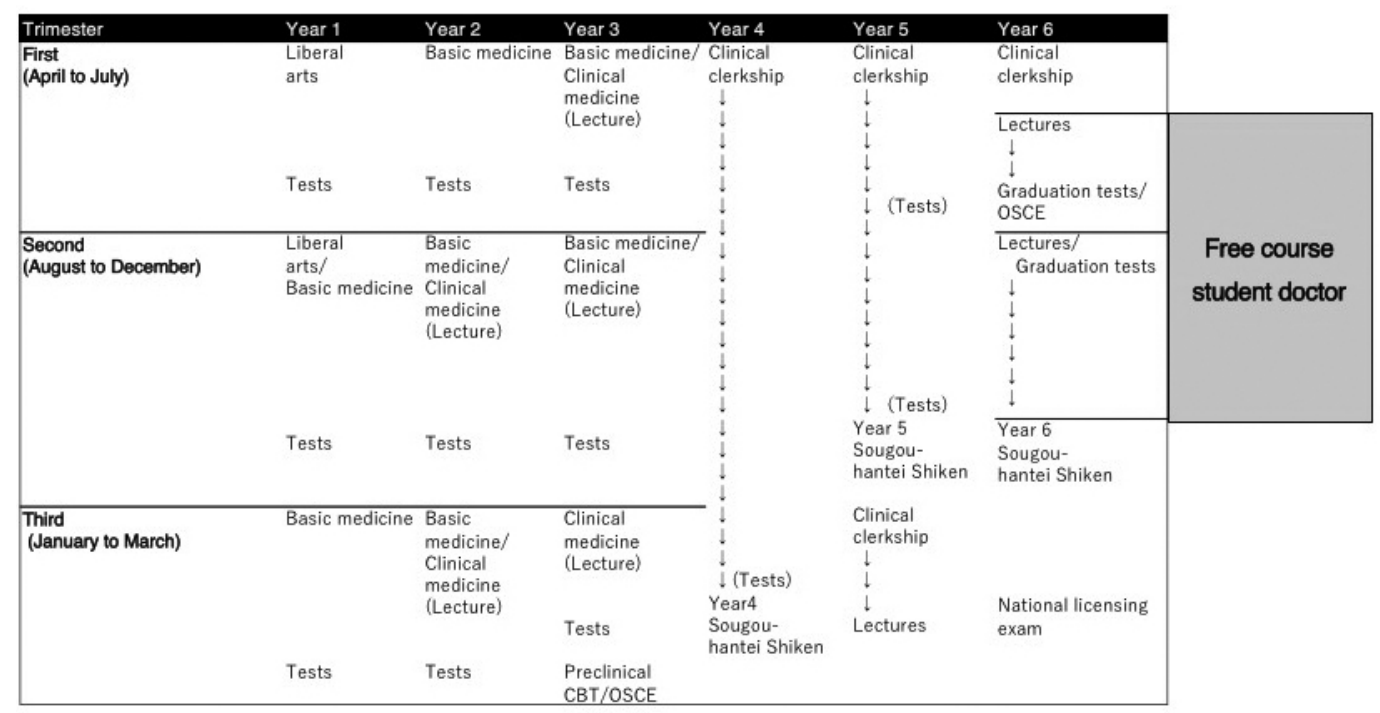

Note. CBT - Computer-based test; OSCE - Objective Structured Clinical Examination

With the help of mentors who were also chosen in accordance with student requests, students can individually design seven-month plans pertaining what subjects, which institutions, when, and how long to study. FCSD participants (FCSDs) are asked to report their learning activities via e-mails on a weekly basis and they receive formative feedback mainly from mentors. Of the 79 students eligible since its implementation, 59 participated in the FCSD while 20 chose to continue the conventional Year 6 curriculum. Of these 59, we examined the learning experiences of 39 students who participated in the FCSD from 2011 to $2015^{23}$. Results showed that the FCSDs successfully selected subjects that they wanted to study and found institutions based on their preference. All participants selected work-based learning in a variety of departments and institutions. They received feedback individually from 
corresponding doctors in the medical practice and also formative feedback in weekly reports from their mentors. All but one selected Japanese institutions not only in medical universities but also in hospitals and clinics, while 30 of 39 FCSDs studied in non-East Asian countries (mainly Western countries). As a rule, the FCSD students were fully exempt from lecturebased education or summative graduation tests. The schedule of one FCSD participant is noted as an example in Table 3-2.

Table 3-2. The Free Course Student Doctor course: Each student can choose learning subjects, institutions and learning contents by themselves with mentor assistance. They experience work-based learning and receive formative feedback.

Student: A 24-year-old male in the 2014 cohort

Mentor: A chief professor at the department of general internal medicine in Jichi Medical University.

\begin{tabular}{|c|c|c|c|}
\hline Date & Learning subjects & Institutions & Main learning contents \\
\hline May & Emergency medicine & $\begin{array}{l}\text { A public emergency medical center } \\
\text { in the student's home prefecture in Japan }\end{array}$ & $\begin{array}{l}\text { The first aid for various } \\
\text { emergent diseases }\end{array}$ \\
\hline June & $\begin{array}{l}\text { (1) Infectious diseases } \\
\text { (2) General internal medicine }\end{array}$ & $\begin{array}{l}\text { (1) Jichi Medical University } \\
\text { (2) A private rural hospital in Japan }\end{array}$ & $\begin{array}{l}\text { (1) In-patient management } \\
\text { (2) Management for common } \\
\text { diseases }\end{array}$ \\
\hline July & Clinical anatomy & Jichi Medical University & Anatomy practice \\
\hline August & Intensive care & A public medical university in Japan & Advanced intensive care \\
\hline September & $\begin{array}{l}\text { (1) General internal medicine } \\
\text { (2) Ultrasonography }\end{array}$ & $\begin{array}{l}\text { (1) A private hospital in Tokyo } \\
\text { (2) Jichi Medical University }\end{array}$ & $\begin{array}{l}\text { (1) Management for common } \\
\text { diseases } \\
\text { (2) Practical skills for } \\
\text { ultrasound examinations }\end{array}$ \\
\hline October & General internal medicine & Jichi Medical University & $\begin{array}{l}\text { Management for complicated } \\
\text { cases }\end{array}$ \\
\hline November & Family medicine & $\begin{array}{l}\text { The department of family medicine in } \\
\text { a medical university in the US }\end{array}$ & $\begin{array}{l}\text { Total health care, the role of } \\
\text { family physicians in the US }\end{array}$ \\
\hline
\end{tabular}

These results indicate that the learning context of FCSD is far removed from the conventional teacher-centered curriculum prevalent in East Asian medical universities. Moreover, we believe the FCSD context corresponds to principles of learner-centered learning proposed by Brandes and Ginnes ${ }^{24}$. First, decision making in all their learning plans entails learners' active participation and high responsibility in their own learning. Second, student-designed plans and formative feedback involving preferable tutors are useful platforms to promote teacher's role as facilitator. Third, full exemption from lectures, written tests, and various opportunities in work-based learning ensures learners' integrative experience that stimulates not only cognitive but also affective domains. 


\section{Participants and design}

In this study, we focused on FCSDs to explore change in self-regulation on self-study in 7 months of the learner-centered context. We also enrolled students eligible for the FCSD program but elected to remain in the conventional teacher-centered Year 6 curriculum (non-FCSDs), because we believed the comparison between these two cohorts was needed to certify the effects of the FCSD context on SRL.

We employed FCSDs and non-FCSDs between 2015-2017 who were in Year 6, post-graduate year (PGY) 1 and 2 during the research period for this study. We excluded participants from before 2015 because recalling learning experience from over 2 years ago was considered problematic.

We invited them to participate in a focus group via e-mail, and all those who agreed were enrolled. We continuously asked all candidates (30 FCSDs and 10 non-FCSDs) to participate until we received agreement or denial of enrollment from them. Eventually, in the first iteration of the focus groups, five FCSDs (four PGY 2 doctors and one PGY 1 doctor), and four nonFCSDs (four PGY 2 doctors) were enrolled. In the second iteration, four FCSDs (four Year 6 students) and three non-FCSDs (two Year 6 students and one PGY 2 doctor) were enrolled. In the third iteration, four FCSDs (four Year 6 students) were enrolled.

\section{Materials}

We collected qualitative data due to the following reasons. First, the study was conducted in a teacher-centered East Asian culture ${ }^{14-15,19}$, so it was difficult to employ a sufficient number of students, who were surely in learner-centered contexts other than the FCSD, for quantitative study. Second, qualitative research is best suited to developing a detailed understanding of a central phenomenon of study difficult to transform into variables ${ }^{25}$. Therefore, we believed that a qualitative approach could more vividly clarify the contrast in learners' SRL between those with a contextual change from a teacher-centered to a learner-centered learning and those staying at the teacher-centered curriculum than a quantitative approach.

In recent years, several scholars have emphasized the significance of objective and real-time process-oriented assessment methods such as microanalysis rather than self-recollection or selfassessment procedures to explore SRL ${ }^{26,27}$. However, we thought real-time assessments might interfere with self-study because participants, especially in teacher-centered culture, might feel pressure or even pretend to do well during assessment. We intended to keep the FCSD context away from assessment-dominated cultures, therefore, used data collection methods in a retrospective manner. 


\section{Procedures}

Focus groups maximize the enrichment of expression and exchange of information on mutual topics, particularly when degree of familiarity with the topic is uniform and power relations between the participants are weak ${ }^{28}$. Therefore, we found focus groups suitable to acquiring qualitative data from groups in which students underwent the same learning activity (self-study of clinical knowledge) in the same setting (contextual transition or continuance).

The FCSDs and non-FCSDs were separately invited to participate in focus groups. Focus groups using PGY 1 and 2 doctors were conducted over Skype ${ }^{\oplus}$, because the participants were busy in their residency programs in different institutions throughout Japan and had difficulty in scheduling face-to-face meetings. Focus groups using only Year 6 medical students took place in a face-to-face manner at JMU. Compared with face-to-face meetings, the internet connection during Skype meetings might influence the frequency of participants statements or verbatim accuracy. However, there was no serious connection problem nor discrepancy between recorded and given statements during Skype meetings.

After informed consent was obtained, a 90-120-minute focus group was conducted. All conversations during the session were recorded and transcribed by research assistants. Participants were not identified in order to guarantee anonymity. The focus group was implemented using three questions prepared beforehand.

Q1. Could you recollect your experience of self-study (of medical knowledge) during the FCSD or the same period in the conventional curriculum?

Q2. During that period, how did you motivate yourself, what strategies did you apply to learning, and how did you assess your understanding?

23. Between before and during the period, did you experience any change in terms of how you motivated yourself and the strategies you applied to learning and to assessing your understanding?

Among the three questions, the third question for FCSDs was considered to be most important to explore changes in SRL when the same learners experience shift from a teacher-centered to a learner-centered context. Rather, purpose of the first and the second question was to prompt FCSDs and non-FCSDs to recall their self-study experience, and articulate three aspects of SRL. These were self-motivation, learning strategies, and metacognition ${ }^{5}$ during the FCSD course, or the same period of didactic lectures and summative tests, respectively.

In focus groups, we have specifically inquired self-study for medical knowledge as a learning activity for the following reasons. First, knowledge acquisition is a common task for students of 
the two groups compared in this study. Second, our previous study ${ }^{10}$ used the similar learning content and successfully illuminated the differences in SRL between the teacher-centered curriculum and the postgraduate rural setting.

\section{Analysis}

From a constructivist paradigm in which 'reality' is subjective and context-specific, and multiple truths are constructed by and between people ${ }^{29}$, we employed constructivist thematic analysis, which examines 'the ways in which events, realities, meanings, experiences and so on are the effects of a range of discourses operating within society" ${ }^{30}$. We viewed this method as suitable for analysis of data from the focus groups, where discourse takes place among participants in the same learning context.

We inductively coded anonymized transcripts of the Japanese scripts from the two groups. Initial coding was conducted by the two lead Japanese researchers, a medical educator (YM) and an education psychologist (MN). Both were experienced in the conduct of qualitative studies relevant to SRL. The analysis was conducted in accordance with the six phases of Braun and Clarke's thematic analysis ${ }^{30}$. The transcripts were thoroughly read and analyzed using an inductive coding approach until agreement on coding was achieved through Skype meetings between the pair.

In the coding process, we utilized terms described in the Motivated Strategies for Learning Questionnaire (MSLQ) ${ }^{31}$. MSLQ is composed of 81 items which quantify the scales of nine types of SRL strategies (rehearsal, elaboration, organization, critical thinking, metacognitive self-regulation, time and study environment, effort regulation, peer learning, and help seeking), and six variables of motivation states (intrinsic goal orientation, extrinsic goal orientation, task value, control of learning beliefs, self-efficacy for learning and performance, and test anxiety).

In initial coding, we firstly coded participants' transcripts for Q1 and Q2 in each group by focusing on how self-motivation, behaviors and reflection took place during the FCSD and the conventional curriculum. Second, we coded their verbatim for Q3 in each group by focusing on how participants in each focus group perceived the changes in self-motivation, behaviors, and reflection before and during the seven months.

After coding agreement, codes and representative statements were translated into English by an American professor living in Japan, who speaks both English and Japanese (AJL). In the final phase, the other authors (HO in Japan and JL and CV in the Netherlands) joined the discussion. We compared codes between students who experienced the shift from teacher-centered to FCSD context and those in the same school year who continuously remained in the teacher-centered curriculum, and a higher-level synthesis of the codes eventually resulted in major themes. 
Results

The result section is structured according to the research questions. Findings are noted with representative statements from focus groups and their reference numbers (e.g. P3-77).

\section{Does change from a teacher-centered to a learner-centered context stimulate SRL?}

To address the first research question, we focused on FCSDs' perceptions toward changes in selfmotivation, behaviors, and reflection between before and during the seven months. These were mainly articulated in focus groups for Q3, or as the question: 'Between before and during the period, did you experience any change in terms of how you motivated yourself, and the strategies you applied to learning and to assessing your understanding?'

While recalling the seven months during the FCSD, FCSDs looked back on their previous selves before entering the FCSD. They perceived themselves as part of a group of elite test takes, who were preprocessed with the teacher's assessment standard, or yardstick. Then, they described contextual changes experienced in the FCSD as liberation or no yardstick, which resulted in confusion.

'There's no yardstick to measure your outcomes. We're all part of that group of elite test takers, so at the beginning when you're liberated from that framework, it's really mind-boggling, confusing.' (FCSD, $\mathrm{P}_{3}-77$ )

However, the FCSDs strived to find an alternative indicator by measuring distance between their current ability and their future self-image. To measure this distance, on one hand they actively employed self-reflection to recognize their current status, and on the other autonomously created their achievable self-image. The FCSDs searched for hints that would help them realize their achievable self-image by employing careful and attentive observation of model doctors and an active approach to communicating with mentors in weekly formative feedback (help seeking in MSLQ).

'I thought I'd find a doctor who could be a model for me, who knew how to write really good patient reports and was really good with them on a one-on-one basis, because I knew there just had to be one like that.' (FCSD, P1-65)

At the same time, they focused on learning strategies used by model professionals and attempted to adapt them to their own self-study.

'I could also see the profs screwing-up sometimes and getting anxious about their errors, and then them talking about what actions to take from then on, which showed me how to overcome mistakes, just something to emulate.' (FCSD, P2-99) 
When completing the FCSD, they began to perceive themselves actively seeking learning strategies used by model doctors or mentors, and adapted them to their self-study. They no longer had to rely on the teacher's yardstick like test scores or pass/fail test results. Aside from a simple memorizing strategy, they began to apply a variety of learning strategies for what they perceived in their patient care or how admirable mentors and medical doctors prepared for patient care.

'As if doing actual treatment, in my case I kind of think how I could do it, looking at results from clinical questions and checking the literature, which is different from until I was a six year.' (FCSD, P3-51)

'I' $m$ writing down summaries of all patients' info on my own, and then making my own plans for the basic treatment for them (in my mind). I'm glad my plans are the same as the professors actually did, and seeking feedback by myselfif I'm wrong.' (FCSD, P-3-19-1).

These changes perceived by FCSD participants were made clear when we referred to perceptions of non-FCSD participants toward Q3. The non-FCSD participants perceived limitations with learning strategies like rote memorization while they continuously stayed in the teacher-centered curriculum.

'It's not like I'm such a bookworm, but in the end, success meant becoming like the textbook.' (Non-FCSD, N2-32)

The non-FCSDs needed to rely on the 'absolute' indicator of test scores or correctness of answers corresponding to teachers' instruction.

'The only way I could figure out if I was learning anything was from exam and practice exam results, then going over material that I got wrong.' (Non-FCSD, N2-28)

All in all, the FCSDs perceptions indicate contextual shift from a teacher-centered to a learnercentered program might improve self-reflection without too much dependence on test scores and increase diversity of learning strategies.

\section{How does SRL develop during transition from a teacher-centered to a learner-centered context?}

We further explored by focusing more on contrast of three elements in SRL between those who experienced the transition and those who did not. We thoroughly reviewed codes from Q1 to Q3, and eventually we identified coherent and meaningful patterns in codes based contrasts between FCSDs and non-FCSDs. Codes were converted into three themes: 1) a motivational contrast between "as an individual with a future self-image" and "as one among a crowd set by 
the teacher's yardstick"; 2) reflection on "between current and future selves" or "between selves and the teacher's yardstick", and 3) diverse or monotonous/homogeneous learning strategies.

Theme 1. Motivational contrast between "as an individual with a future self-image" and "as one among a crowd set by the teacher's yardstick"

Overall, the most prominent feature of the FCSDs was an enriched description of 'selves' from the past to present and future, as an individual learner. The FCSDs described relevance between their past and present learning activities and their future professional roles.

'I just imagined myself going around in a group, just one among many, but then I began to take-off as an individual... the biggest change was that I began thinking that how far I want to go was really up to me, so then I could go and make the choices for my future.' (FCSD, P2-44)

Their self-motivation reached a climax when FCSDs perceived themselves being treated as a responsible person on the same level in learning by mentors and surrounding professionals in medical practice.

'In the Free Course it was like I was given a lot of responsibility by the teachers which really motivated me.' (FCSD, $\left.\mathrm{P}_{1-38}\right)$

On the other hand, the non-FCSDs were stuck in their performance within the values set by the teacher's yardstick (e.g. assessment test scores, and pass/fail standards) and described themselves as 'someone", resulting in the scarcity of future self-image as a doctor.

'It was more like I was someone on a mission, rather than, you know, wow, I wonder what it would be like to work as a doctor.' (Non-FCSD, N1-36)

They stated fear of failing tests strongly motivated them to undertake self-study. However, fearbased motivation only prompted them to seek the 'safety zone', where they could perceive themselves not left behind other classmates in a crowd set by the teacher's yardstick (pass/fail threshold).

'It's a safety zone. Since there's no getting out of taking exams, I really only focused on placing in the "non-fail" range, not on getting a high score.' (Non-FCSD, N1-39)

Theme 2. Reflection on "between current and future selves" or "between selves and the teacher's yardstick"

In the FCSD course, liberation from the absolute indicator set by the teacher's yardstick eventually helped them identify an alternative indicator: distance between their current ability and achievable self-image. The FCSDs recalled a possible alternative indicator during self-study 
in the FCSD context. They attempted to set 'their own indicator' within themselves, for example, by measuring the smoothness of their medical practice in a self-reflective manner.

'From the outset, going from the first-time patient interview to the assessment...I was able to get the hang of it compared to before, and at the same time I kept reviewing how smoothly I interviewed her or how I was nervous and skipped some steps. (FCSD, P2-42)

On the other hand, non-FCSDs also had reflective-like behaviors in their self-study but they did not perceive they needed to carefully evaluate their learning outcomes in a self-reflective manner or attempted to establish their own concrete indicators for their achievements. They seemed to blindly rely on referring to test score or pass/fail results determined by teachers.

'Well, what I usually did for better or for worse was kind of rely on my gut feelings, or else, you know, like test scores.' (non-FCSD, N2-34)

Theme 3. Diverse or monotonous/homogeneous learning strategies.

In the teacher-centered context, undergraduates associated effort management for memorizing knowledge prepared by teachers with test success or at least survival. They studied using effort management on repeated memorization of textbooks or handouts from teachers, and sometimes they were demotivated by overwhelming memory workload.

'There were questions about surgery...but ultimately there was a lot of material I just didn't get and couldn't prepare for, so the next tests are going to be hell...no matter how much I looked at my textbook things just didn't click...overall, I just couldn't jump-start my motivation so I ended-up just ignoring a whole lot.' (Non-FCSD, N2-14)

After completing the FCSD, they perceived the diversification of their learning strategies while undertaking drill exercises using test items with clinical vignettes. Instead of merely reproducing the information written in textbooks, or lecture handouts, they came to associate clinical vignettes with what they encountered or what model physicians experienced in real clinical practice (elaboration in MSLQ). They mentioned that they were eventually able to deepen their understanding of the relevant structured knowledge (organization in MSLQ). While answering test items during self-study, they began to convert the negative feeling of mistakes into the acceptance as a next learning subject, that could be referred to as control of learning beliefs in MSLQ.

'Before it was like, I'd be figuring out problems (in test items), I know that, I don't know that, but now I have a much clearer idea of how I'm getting things wrong, I can analyze it... So now making mistakes is not so much of a big thing. If it happens, it's like, ok, let's just pay more attention next time.' (FCSD, P2-67) 


\section{Discussion}

To our knowledge, this is the first study specifically documenting contrast in SRL elements between undergraduates experiencing the contextual change from a teacher-centered to a learnercentered learning and those continuously remaining in a teacher-centered context. By incorporating the results of qualitative analysis for the two research questions, we concluded that learner-centered contexts could promote 1) motivational shift from "one among a crowd set by the teacher's yardstick" to "an individual with a future self-image"; 2) reflection comparison from "between selves and the teacher's yardstick" to "between current and future selves"; and 3) strategies from monotonous/homogeneous (memorization) to diverse (elaboration, organization, control of learning beliefs etc.) (Figure 3-1). We found the possible link between formation of individual identity as an independent learner and eventual development of self-reflection and diverse learning strategies. Some theories may explain the linkage of identity formation and motivation-driven self-reflection and strategic learnings.

Figure 3-1. Changes in self-regulated learning from a teacher-centered (non-FCSDs) to a learner-centered (FCSDs) context in undergraduate medical education

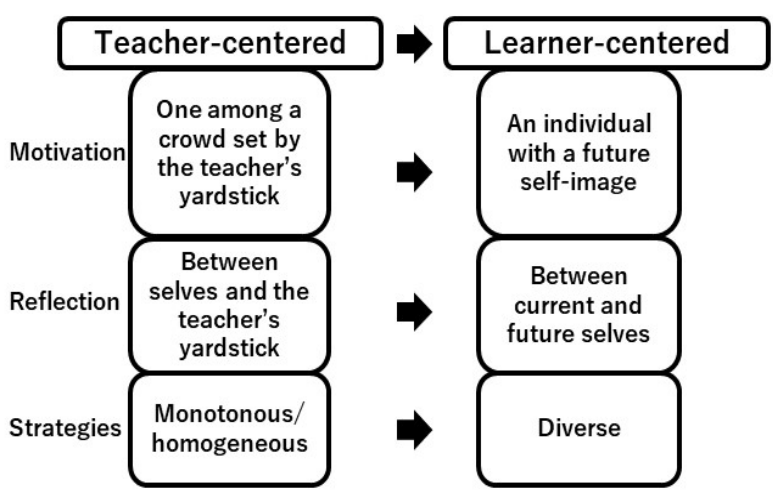

First, we employed the "constructive developmental theory" proposed by Kegan ${ }^{32}$, which describes the process of identity formation and subsequent behavioral changes. According to Kegan, identity formation is composed of five stages, of which the second to fourth stages are particularly relevant to the learning period from undergraduate to postgraduate study in medicine $^{33}$.

At Stage 2, medical students still lack a broader understanding of what it means to be a physician, and their motivation and performance is based on a narrowly defined and superficial understanding. As they move to Stage 3, they begin to internalize social expectations, behaviors, and values of the profession, and become sensitive to whether they are doing things right as a 
physician. This emerging identity motivates them to learn rules of appropriate action and to look to authority figures for direction and reassurance that they are doing well and fitting in. In Stage 4 , individuals construct a personal system of values and internal processes they use to evaluate external messages about their role and competence. Through this evaluation, they acquire the ability to think about themselves in relation to the larger system involving all medical professionals. The transition from stage to stage is not gradual but rather precipitated by emerging "identity crises" ${ }^{4}$. Namely, when faced with discrepancies between their understanding of themselves in the role and their understanding of experiences and challenges they are facing, they begin to reevaluate their situation, incorporate new information, and eventually develop a new understanding of the world or themselves ${ }^{33}$.

In our present study, all the FCSDs articulated discomfort and anxiety of being exempt from teacher-centered values. On entering the FCSD context, they were accustomed to pursuing common learning goals set by the teacher's yardstick, assuring they did not differ from classmates in order not to fail. However, the FCSD context pushed them to face discrepancies between the role of "as one among a crowd set by the teacher's yardstick" and the challenge of having no prepared goals or assurance of their improvement in self-study. They recognized the necessity of finding alternative indicators within reach of their perception to assure themselves they were doing things right without making comparisons "between selves and the teacher's yardstick." Accordingly, they began to reflectively compare "between current and future selves." In other words, such a crisis prompted them to ask themselves who they would like to be as an individual professional. While overcoming the discrepancy, they were likely to internalize how authority figures (mentors and role-models) behave by incorporating new learning strategies. This could result in diversification of learning strategies. Cruess et al. ${ }^{35}$ emphasized importance of individual identity formation in medical education by referring to 'professional identity formation (PIF)', defined as formation of "a representation of self, achieved in stages over time during which the characteristics, values, and norms of the medical profession are internalized." PIF results in an individual thinking and acting over what they want to learn and what they find important in a clinical environment ${ }^{35}$. All in all, these notions support contextual change toward learner-centered learning caused motivation to be based on the idea of an individual with a future self-image, and reflective comparison to be oriented to current and future selves. Accordingly, learning strategies were no longer limited by the teacher's yardstick, and became diverse.

Second, the "self-determination theory" proposed by Ryan and Deci ${ }^{36}$ could be employed to explain how the FCSD context promoted a shift in regulation of learning from controlled to autonomous. This theory states the degree an individual's behavior is self-motivated depends on fulfillment of intrinsic needs for competence, autonomy, and psychological relatedness. In our present study, the FCSDs perceived the most advantageous aspect of their approach was the ability to decide one's own learning plan and the opportunity to choose a tutor they admired and an institution where respected physicians work. Moreover, their self-motivation reached a climax 
when students perceived themselves as being treated by mentors and surrounding professionals as similarly responsible in learning. These features fulfill the need for autonomy (selfdetermination in learning activities), competence (being treated as a responsible person), and relatedness (close interaction between admired tutors and learners), and eventually made students more self-motivated.

In practice, the idea that contextual change from a teacher-centered to a learner-centered (individualized) learning positively influences SRL could be used as follows. We propose the undergraduate curriculum be designed in such a way that students more closely participate in planning of their content by self-determination with higher responsibility. The higher responsibility entailed by self-determination for their own learning might encourage them to think of their own learning activities more carefully and profoundly. Instead of having their learning outcomes all designed by teachers, they could develop their learning outcomes based on their reflection of how they would like to be in the future, and how they have missed learning in the past. From these points of views, the FCSD at JMU and self-proposed studentselected components in the $\mathrm{UK}^{37}$ might be a good platform to give opportunities fostering PIF and SRL.

Of course, the undergraduate curriculum should certify the mastery of certain knowledge and skills. Because medical students are inaccurate in self-judgement of their knowledge, skills and performance ${ }^{2}$, feedback is inevitable. One study found that individualized and narrative descriptive feedback from mentors promotes PIF elements ${ }^{38}$. Therefore, to optimize selfdetermination-oriented elective courses, we need to establish mentorship systems to provide individualized and narrative descriptive feedback on a regular basis. To maximize the effect of feedback, the ability of students as well as mentors to communicate with each other should be fostered sufficiently.

\section{Limitations and further research}

A limitation of this study is its analytic comparison between two groups, which were each sufficiently competitive to pass the national licensing exam at the end of the second-to-last school year. However, the findings in this study would justify further investigation to explore whether a curriculum reform toward learner-centered learning could stimulate SRL in low-grade undergraduates, especially in teacher-centered education culture.

A second limitation is this study only investigated the retrospective notion of learning activities. We admit the possible uncertainty of qualitative data collected from participants' recollection. However, both cohorts were composed of participants with higher grades than the average in the Year 5 recollection-dominated tests, and we only included those participating in the FCSD or the counterpart in the conventional curriculum within the latest two years in order to maximize the accuracy of recollection. Moreover, the contrast in SRL changes 
between those experiencing the contextual change and remaining in the teacher-centered curriculum ensures this contextual change could promote significant changes in SRL over the seven months.

A third limitation is we did not directly evaluate SRL levels when they started the FCSD or decided to stay in the teacher-centered curriculum. Even though changes of SRL were identified between before and during the FCSD according to the focus groups statements for Q3, the present study design might leave the assumption FCSDs chose this student-selected elective course because they were self-motivated to enter new challenging environments to develop as individual learners.

Judging from the second and third limitation, a more valid approach to the research question can be to compare the SRL levels of the same individuals among pre-, peri-, and post-FCSD. Further investigation should be conducted in such a longitudinal manner.

A fourth limitation is that this study only focused on self-study for knowledge acquisition while a variety of learning activities take place in undergraduate settings. Self-regulation in learning is applied not only to self-study but also to learning in groups. Recent theories suggest that self-regulation in learning can be developed through social transactions, considered the central core of regulated learning ${ }^{3,4,8}$. In the context of our present study, for instance, undergraduates might develop SRL in a peer-group study rather than by self-study. Accordingly, future studies should focus on changes in regulation for learning through social interactions among participants in various learning settings.

\section{Conclusions}

Allowing for these limitations and the need for further research, this study indicates contextual change toward learner-centered learning could promote SRL even in students strongly accustomed to teacher-centered learning. In the learner-centered context, students began to construct their future self-image, conduct reflection on current and future selves, and seek diverse learning strategies by referring to future 'self' models.

\section{List of abbreviations}

SRL: Self-regulated learning; PBL: Problem-based learning; FCSD: Free Course Student Doctor; MSLQ: Motivated Strategies for Learning Questionnaire; PIF: Professional identity formation 


\section{References}

1. Frank JR. The CanMED 2005 Physician Competency Framework: Better Standards, Better Physicians, Better Care. Ottawa, Canada: Royal College of Physicians and Surgeons of Canada; 2005.

2. Sandars J, Cleary TJ. Self-regulation theory: application to medical education: AMEE Guide No 58. Med Teach. 2011; 33: 875-86.

3. Brydges R, Butler D. A reflective analysis of medical education research on self-regulation in learning and practice. Med Educ. 2012; 46: 71-9.

4. Berkhout JJ, Helmich E, Teunissen PW, Van der Vleuten CP, Jaarsma ADC. Context matters when striving to promote active and lifelong learning in medical education. Med Educ. 2018; 52: 34-44.

5. Zimmerman BJ. A social cognitive view of self-regulated academic learning. Journal of Educ Psychol. 1989; 81: 329-39.

6. Artino AR, Jones KD. AM Last Page: Self-regulated learning — a dynamic, cyclical perspective. Acad Med. 2013; 88: 1048.

7. Zimmerman BJ: Attaining self-regulation: A social-cognitive perspective. In Handbook of SelfRegulation. Edited by: Boekaerts M, Pintrich P, Ziedner M. San Diego, CA, USA: Academic Press; 2000: 13-39.

8. Rich JV. Proposing a model of co-regulated learning for graduate medical education. Acad Med. 2017; 92: 1100-4.

9. Van Houten-Schat MA, Berkhout JJ, Van Dijk N, Endedijk MD, Jaarsma ADC, Diemers AD. Self-regulated learning in the clinical context: a systematic review. Med Educ 2018; 52: 1008-15

10. Matsuyama Y, Nakaya M, Okazaki H, Leppink J, Van der Vleuten C. Contextual attributes promote or hinder self-regulated learning: A qualitative study contrasting rural physicians with undergraduate learners in Japan. Med Teach. 2018; 40: 285-95.

11. Kim KJ, Jang HW. Changes in medical students' motivation and self-regulated learning: a preliminary study. Int J Med Educ. 2015; 6: 213-5.

12. Chitkara MB, Satnick D, Lu WH, Fleit H, Go RA, Chandran L. Can Individualized Learning Plans in an advanced clinical experience course for fourth year medical students foster SelfDirected Learning?. BMC Med Educ. 2016; 16: 232.

13. Quirk ME, Harden RM. Curriculum planning and development. In A Practical Guide for Medical Teachers 5th edition. Edited by: Dent J, Harden R, Hunt D. London, UK: Elsevier, 2017: 4-12.

14. Yoshioka T, Suganuma T, Tang AC, Matsushita S, Manno S, Kozu T. Facilitation of problem finding among first year medical school students undergoing problem-based learning. Teach Learn Med. 2005; 17: 136-41.

15. Frambach JM, Driessen EW, Chan LC, Van der Vleuten CPM. Rethinking the globalisation of problem-based learning: How culture challenges self-directed learning. Med Educ. 2012; 46: 738-47.

16. Ziguras C. Educational technology in transnational higher education in South East Asia: The cultural politics of flexible learning. Educ Tech Society. 2001; 4: 8-18.

17. Waterval D, Tinnemans-Adriaanse M, Meziani M, Driessen E, Scherpbier A, Mazrou A, Frambach J. Exporting a Student-Centered Curriculum: A Home Institution’s Perspective. J Stud Int Educ. 2017; 21: 278-90.

18. Nisbett R. The Geography of Thought: How Asians and Westerners Think Differently... Why. New York, NY, USA: Free Press; 2003. 
19. Ho DYF, Peng S, Chan FS: Authority and learning in Confucian-heritage education: a relational methodological analysis. In Multiple Competencies and Self-Regulated Learning: Implications for Multicultural Education. Edited by: Salili F, Chiu SY. Greenwich, CT, USA: Information Age Publishing; 2001: 29-47.

20. Tweed RG, Lehman DR. Learning considered within a cultural context: Confucian and Socratic approaches. Am Psychol. 2002; 57: 89-99.

21. Kwok P. Examination-oriented knowledge and value transformation in East Asian Cram Schools. Asia Pac Educ Rev. 2004; 5: 64-75.

22. Jichi Medical University: Toukei data (Japanese). 2018 [https://www.jichi.ac.jp/medicine/about/ statistics.html]. Accessed 15 April 2018.

23. Matsuyama Y, Okazaki H, Kario K, Noda Y. Poster: Self-directed and internationalized scopes among participants in the Free Course Student Doctor system - a unique student-selected bedside training program at Jichi Medical University [abstract]. Barcelona: Association of Medical Education in Europe (AMEE); 2016.

24. Brandes D, Ginnes P. A Guide to Student-Centred Learning. Oxford: Basil Blackwell; 1986.

25. Creswell JW. The process of conducting research using quantitative and qualitative approaches. In Educational Research. 4th ed. Edited by: Creswell JW. Boston, USA: Pearson; 2012:15-70

26. Gandomkar R, Sandars, J. Clearing the confusion about self-directed learning and self-regulated learning. Med Teach. 2018; 40: 862-63.

27. Cleary T. Emergence of self-regulated learning microanalysis. In Handbook of self-regulation of learning and performance. Edited by Schunk DH, Zimmerman BJ. London, UK: Routledge; 2011: 329-45.

28. Stalmeijer RE, Mcnaughton N, Van Mook WN. Using focus groups in medical education research: AMEE Guide No. 91. Med Teach. 2014; 36: 923-39.

29. Bergman E, De Feijter J, Frambach J, Godefrooij M, Slootweg I, Stalmeijer R, Van der Zwet J. AM last page: A guide to research paradigms relevant to medical education. Acad Med 2012; 87: 545.

30. Braun V, Clarke V. Using thematic analysis in psychology. Qual Res Psychol. 2006; 3: 77-101.

31. Pintrich PR, Smith D, Garcia T, McKeachie WJ. A manual for the use of the Motivated Strategies for Learning Questionnaire (Technical Report 91-B-004). Michigan, USA: The Regents of the University of Michigan; 1991.

32. Kegan R. In over our heads: The mental demands of modern life. Cambridge, MA, USA: Harvard University Press; 1994.

33. Jarvis-Selinger S, Pratt DD, Regehr G. Competency is not enough: integrating identity formation into the medical education discourse. Acad Med. 2012; 87: 1185-90.

34. Erikson EH. The Life Cycle Completed. NewYork, NY, USA: Norton; 1982.

35. Cruess RL, Cruess SR, Boudreau JD, Snell L, Steinert Y. Reframing medical education to support the development of a professional identity. Acad Med. 2014; 89: 1446-51.

36. Ryan RM, Deci EL. Self-determination theory and the facilitation of intrinsic motivation, social development, and well-being. Am Psychol. 2000; 55: 68-78.

37. Murphy MJ, De A Seneviratne R, Remers OJ, Davis MH. Student selected components: studentdesigned modules are associated with closer alignment of planned and learnt outcomes. Med Teach. 2009; 31: e489-93.

38. Camp CL, Gregory JK, Lachman N, Chen LP, Juskewitch JE, Pawlina W. Comparative efficacy of group and individual feedback in gross anatomy for promoting medical student professionalism.

Anat Sci Edu. 2010; 3: 64-72. 
Chapter 4

Education in professional identity formation-oriented enhances self-regulated learning: a mixed-method study from a community-based clinical clerkship in Japan 


\section{Abstract}

Background: Previous studies indicate professional identity formation (PIF), or formation of self-identity with internalized values and norms of professionalism, might influence selfregulated learning (SRL). However, it remains unclear whether or not PIF-oriented education can improve SRL in medical students.

Methods: Rural community-based clinical clerkships (rCBCC) for Year 5 (Y5) students in Jichi Medical University (JMU) provide a learning environment where undergraduates will work in future with alumni directly mentoring them. To make rCBCC more PIF-oriented, we created a learning platform using Google Forms and a PIF-oriented teaching manual for mentors. These were designed to help undergraduates articulate future 'self' images as professionals, and have in-depth communication with mentors as role models pertaining to medical professionalism. Forty-one Y5 JMU students experienced PIF-oriented rCBCC, while 41 Y5 control subjects experienced the original format rCBCC. Changes in SRL between the two groups were compared using 15 categories of motivated strategies for learning questionnaire (MSLQ). We also explored how the PIF-oriented rCBCC changed their SRL by thematic analysis of questionnaire and interview data.

Results: A moderate improvement of intrinsic goal orientation $\left(p=0.005, \varepsilon^{2}=0.096\right)$ and a mild improvement of critical thinking ( $\left.p=0.041, \varepsilon^{2}=0.051\right)$ were observed in the PIF-oriented rCBCC. Qualitative analysis revealed they started viewing 'professional responsibility' as selfstudy motivator. Awareness of 'responsibility' led to pursuit of authenticity and medical knowledge applicability, and fostered critical thinking about learning contents. They also began elaborating learning contents in line with processes.

Conclusion: This is the first study showing some effects of PIF-oriented education on SRL improvement. Fostering PIF may be important for stimulating learners' intrinsic SRL behaviors.

Key words: Self-regulated learning, Professional identity formation, Community-based clinical clerkship, Rural medicine 


\section{Backgrounds}

As clinical knowledge advances rapidly, medical professionals need to update knowledge autonomously throughout their professional life. Therefore, self-regulation in life-long learning has become an important competency $y^{1-4}$.

Self-regulated learning (SRL) is defined as learners' active participation in their own learning process from metacognitive, motivational, and behavioral perspectives ${ }^{5}$. Zimmerman described SRL as a cyclical process composed of three phases. In the forethought phase, students set learning goals and choose strategies for attaining goals. In the performance phase, students monitor and control behavior to attain goals. In the self-reflection phase, students formulate new learning goals and strategies for future similar situations $s^{6,7}$.

Because postgraduate medical training cannot prepare learners for every challenge their qualification bring $\mathrm{s}^{1,8}$, lack of readiness to engage in SRL following primary medical education is problematic ${ }^{3,8,9}$.

\section{Contextual factors influencing SRL}

Thus far, several studies have emphasized learning context determines learner engagement in SRL. Brydges and Butler ${ }^{3}$ summarized contextual factors influencing SRL: At the broadest level from cultural and social learning expectations; and within learning environments from pedagogical approaches, specific assignments, learning support, and types of feedback or evaluation. Van Houten-Schat et al. ${ }^{9}$ specifically explored contextual factors influencing SRL in the clinical environment, such as available time, characteristics of learning environment (work climate, engagement in team), and patient-related factors.

Matsuyama et al. investigated contextual factors promoting or hampering SRL in medical knowledge self-study ${ }^{10,11}$ among Japanese medical students. According to these studies, identity formation as a future unique professional could promote student self-oriented reflection and acceptance of various learning strategies in a self-regulated manner. Oppositely, teacher-centered and test-oriented contexts in undergraduate education may keep students overly-dependent on teacher rubrics and hamper developing self-image as unique professionals. All in all, their conclusions suggest professional identity formation (PIF) could be a key factor fostering SRL.

\section{PIF-oriented education in the undergraduate curriculum}

PIF is defined as formation of 'a representation of self, achieved in stages over time during which the characteristics, values, and norms of the medical profession are internalized ${ }^{\prime 2}$. Cruess et al, advocates of PIF, state it results in individuals considering their clinical environment behavior ${ }^{12}$. 
However, while many studies have explored outcomes of PIF-oriented medical education regarding professional and unprofessional behaviors ${ }^{13-15}$, there are no studies assessing effects of PIF-oriented educational approaches on learning behaviors.

Therefore, we attempted to create a new PIF-oriented education format and measure changes in undergraduates' SRL before and after the PIF-oriented learning experience. We believe educators can acquire evidence for undergraduate-level pedagogies helping students acquire more self-regulated learning behaviors.

\section{The current study}

In this study, we investigated changes in SRL after learners received PIF-oriented education based on Matsuyama et al.'s previous research ${ }^{10,11}$. Results demonstrate awareness of a future unique 'professional self' foster readiness to seek model learning strategies in future practice. Other articles show professional identity robustly forms when role models demonstrate appropriate behaviors and norms for professionalism ${ }^{14-17}$.

By combining these aspects, we created a PIF-oriented education format for clinical year students characterized as follows: students (1) observe mentors in future work settings, (2) articulate their future unique 'self' images to form professional identity, and (3) have in-depth communication pertaining to values and norms of professionalism with their mentors regarding workplace-based learning. We hypothesize PIF-oriented education with these three elements could improve SRL (Figure 4-1).

Figure 4-1 Conceptual framework for the PIF-oriented education format

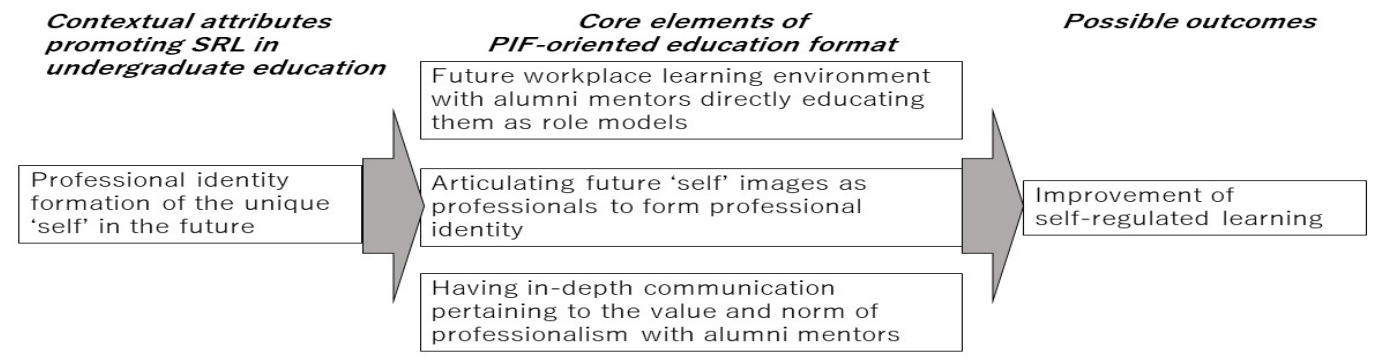

In this study, we verified this hypothesis using a rural community-based clinical clerkship (rCBCC) at Jichi Medical university (JMU) in Japan. Under the JMU policy, two to three applicants per prefecture are admitted to university with full support of tuition fee from the prefectural government for the six-year curriculum in exchange for working in rural settings of their home prefecture from PGY3 to $9^{10,18}$. We chose the rCBCC in Year 5 curriculum as a 
setting for this study because this program allows rural physician-to-be students to study in their future work settings (rural hospitals or clinics in their home prefecture) with mentors who are role models and also JMU alumni rural physicians ${ }^{19}$. In order to promote PIF around rCBCC, we created a PIF-oriented learning platform in which each student articulates their future unique 'self' images, and have in-depth communication pertaining to values and norms of professionalism with mentors. We also made an instruction manual for attending JMU graduates to intensify PIF education during the rCBCC. This version of $\mathrm{rCBCC}$ was named PIF-oriented rCBCC (PIF-rCBCC). We conducted a comparative study between PIF- and the original rCBCC in 2018 and 2019 (Figure 4-2). To clarify the purposes of study, we formulated two research questions.

1. Does PIF-rCBCC improve SRL better than the original format $r C B C C$ ?

2. If so, how does the PIF-oriented rCBCC improve SRL?

We conducted a comparative mixed method study to answer these research questions. This study was approved by the university's ethics committee (reference number: 19-001).

Figure 4-2. Schedule of the present study

Subject selection and informed consent

Data collection from responses to Japanese version of Motivated Strategies for Learning Questionnaire (MSLQ-J)

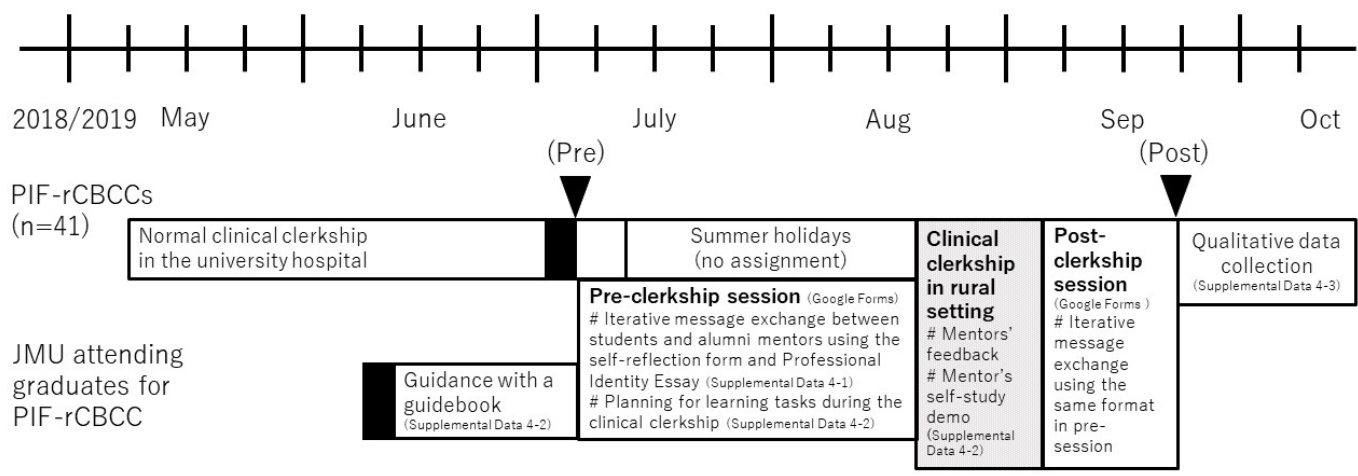

Original rCBCCs

$(n=41)$

Normal clinical clerkship in the university hospital 


\section{Methods}

\section{Settings:}

\section{Jichi Medical University}

Jichi Medical University (JMU) in Japan was founded to educate medical students to become rural physicians. JMU graduates are appointed to a clinic or hospital in medically underserved or geographically-isolated areas during postgraduate years (PGY) 3 to 9 , where consultation with specialists and educational instruction from teachers is difficult ${ }^{10,18}$. Accordingly, they have fewer chances to train their clinical knowledge and skills under systematic instructional programs between PGY 3 and 9. While JMU graduates are supposed to improve medical knowledge and skills in a self-directed manner, JMU undergraduates are not sufficiently ready with SRL for postgraduate settings $s^{10,11,20}$. Therefore, the university educational board introduced a new program aimed at independent learning at Year $6^{11}$, and now the attempt has been expanding across school years. PIF-rCBCC is another attempt to promote better SRL.

\section{The original $\mathrm{CBCC}$}

The original rCBCC was introduced in $1998^{19}$. It takes place for 2 weeks from late August to early September just after the long summer holidays between the first and second trimester in Year 5. During rCBCC, Year 5 students stay in their home prefecture community hospital or clinic where JMU graduates work. Every year, two to five JMU graduates per prefecture are appointed to be attending doctors for $\mathrm{rCBCC}$ based on reputation and motivation for teaching among their prefectural communities. Although JMU faculty organize and manage the program and assure instructional quality, attending JMU graduates determine the program's process. In 2001, standards for learning activities were proposed, which include ambulatory care, home care, hospital care, placement in mobile clinics, on-call work, rehabilitation, health education, health check-ups, vaccination, day services, and placement in welfare facilities (welfare institutions or nursing homes for the aged) ${ }^{19}$.

Before the rCBCCs, students had few chances for sufficient communication with their attending doctors. Following the rCBCC, students were asked to submit a report but did not receive reflective feedback from their mentors. There was no platform to communicate what JMU alumni expected undergraduates to learn, and what undergraduates hoped to learn to become medical professionals in their future setting. Therefore, although the original rCBCC provided an advantageous setting for PIF from learners' future role models (Figure 4-1), we were concerned the lack of communication might interfere with establishing professional identity as rural physicians.

\section{PIF-rCBCC}

In order to make learning contexts of rCBCC more PIF-oriented, we introduced a PIF-oriented communication platform designed in accordance with two other core elements in Figure 4-1: articulating future 'self' images as 'professional' to form professional identity, and having in-depth communication pertaining to values and norms of professionalism with mentors. 
We used an online communication platform or Google Forms because we expected students and mentors to post opinions and feelings frequently with each other. The structure of Google Forms for PIF-rCBCC is described in Supplemental Data 4-1.

In Google Forms, participants were asked to post essays to verbalize their future self-image and receive feedback from mentors. Also, they were asked to fulfill the professional identity essay (PIE) invented by Kalet, et al. ${ }^{21}$. PIE is useful for helping learners articulate their values and norms about medical professionalism from their own point of view and to help teachers provide feedback by referring to rubrics based on Kegan's constructive developmental theory ${ }^{21,22}$. This study used a Japanese version of Kalet et al.'s PIE form originally in English. Back translation between English and Japanese was conducted by the main author (YM, Japanese) and an American professor living in Japan literate in both English and Japanese (AJL).

Prior to PIF-rCBCC, mentors received an instruction manual explaining in detail the aim of PIF-rCBCC and the program from receiving the first student submission on Google Forms in July to the final provision of reflective feedback to students in September (Figure 4-2). The outline of the manual was shown in Supplemental Data 4-2.

Aside from iterative message exchanges in Google Forms before and after the clinical clerkship, students in PIF-rCBCC were asked to create one or more learning themes during rCBCC based on verbalized future self-images and values and norms of medical professionalism. In response to learners' themes, alumni mentors observed their learning and gave just-in-time feedback during the two weeks of rCBCC. Also, mentors showed students how to overcome future workplace challenges through self-study. Aside from these directives, mentors were basically allowed to educate students in their own ways.

After the two-week clerkship, students were asked to re-articulate their own future images as medical professionals re-writing Kalet's PIE and receiving comments from JMU graduates' feedback on them by Google Forms.

\section{Subjects}

As Figure 4-2 shows, we first selected attending rural physicians for PIF-rCBCC. In 2018 and 2019, ninety-four JMU graduates registered as attending graduates for the original rCBCC. Among them, we randomly selected 20 candidates for PIF-rCBCC mentors in 2018 and 2019 and attempted to obtain informed consent for the contribution to PIF-rCBCC in this study. Eventually, 17 and 13 JMU alumni agreed on participation in 2018 and 2019, respectively. Eight alumni participated in both years.

Independent from this study, the JMU Center for Community Medicine matched one alumni with one to three students for the original rCBCC. The thirty PIF-rCBCC alumni were paired with one to three students before informed consent was obtained. In this study, we used students paired with the thirty PIF-rCBCC alumni as candidates for PIF-rCBCC group subjects. There were twenty-two and twenty candidates in 2018 and 2019, respectively. One candidate in 2019 declined the participation. Eventually 41 students were registered as subjects in PIF-rCBCC 
group (PIF-rCBCCs). Simultaneously, 41 original rCBCC participants (original rCBCCs) control subjects were chosen and provided informed. Participants in both groups were paired by gender and academic ranking from the previous year (Year 4), because previous studies have showed gender ${ }^{23,24}$ and academic performance ${ }^{25}$ might independently influence SRL development.

\section{Procedures}

We chose a convergent mixed method for the first research question 'Does PIF-rCBCC improve $S R L$ better than the original-rCBCC?'. A rationale for this method is 'one data collection form supplies strengths to offset the weaknesses of the other form to achieve a more complete understanding of a research problem ${ }^{26}$. We used an explanatory mixed method to address the second research question 'How does the PIF-oriented rCBCC improve SRL?'. A rational for this method is that following qualitative approaches can explain quantitative results ${ }^{26}$. We used this method because we believed qualitative analysis would explain how the PIF-oriented rCBCC improved SRL in quantitative data in detail.

\section{1) Quantitative Approach}

We measured learners' SRL levels by a Japanese-language version of the Motivated Strategies for Learning Questionnaire (MSLQ-J) ${ }^{27}$ before and after subjects participated in PIF-rCBCC or original rCBCC. MSLQ is reported useful measuring SRL in medical undergraduate education ${ }^{28-30}$, and is composed of 81 items with seven-point Likert scales quantifying levels of nine types of SRL strategies (rehearsal, elaboration, organization, critical thinking, metacognitive selfregulation, time and study environment, effort regulation, peer learning, and help seeking), and six variables of motivation states (intrinsic goal orientation, extrinsic goal orientation, task value, control of learning beliefs, self-efficacy for learning and performance, and test anxiety). We used 81 items of MSLQ used in a medical school context ${ }^{29}$. All were translated into Japanese and back translations were made between the main author (YM) and an American professor living in Japan literate in both English and Japanese (AJL).

\section{2) Qualitative Approach}

To explore change of perception regarding motivation, strategies and reflective behaviors in daily self-study before and after the PIF-rCBCC, we created a questionnaire composed of seven questions (shown in Supplemental Data 4-3). In 2019, we also conducted one-on-one interviews after analyzing qualitative data from the questionnaire from 41 participants in 2018 and 2019 to achieve data saturation ${ }^{31}$. We employed three interviewers who are familiar with the original $\mathrm{rCBCC}$ but not engaged in Year 5 students' assessment in order for interviewees to openly articulate their own perceptions. We asked all PIF-rCBCCs in 2019 to join the interviews and twelve students agreed to have interviews. Interviews were conducted in a semi-structured manner using an interview form with similar questions to those in the questionnaire (shown in Supplemental Data 4-3). The interviewers were told by the main author (YM) beforehand to obtain data about change of 
perception regarding motivation, strategies and reflective behaviors. After collecting interview data from 10 students, the two main authors (YM and HO) found no additional meaningful codes emerging and concluded data reached saturation ${ }^{31}$ and stopped interview data collection.

\section{Analysis}

\section{1) Quantitative Approach}

We compared the 15 MSLQ-J pre-course subcategory scores of PIF-rCBCCs and original rCBCCs subjects using Kruskal-Wallis one-way analysis of variance (ANOVA). After confirming there were no statistically significant differences between the two group, we compared subtracted (post-pre) scores in the 15 MSLQ-J subcategories between PIF-rCBCCs and original rCBCCs using Kruskal-Wallis one-way ANOVA. A $p$-value $<0.05$ was considered statistically significant. The effect sizes for comparisons were also calculated using $\varepsilon^{2}$ values where small effect sizes ranged from 0.01 to $<0.08$, medium effect sizes ranged from 0.08 to $<0.26$ and large effect sizes from $\geq 0.26$. We used JAMOVI version 1.0.7.0 ${ }^{32}$ for the statistical analysis.

\section{2) Qualitative Approach}

From a constructivist paradigm in which 'reality' is subjective and context-specific, and multiple truths are constructed by and between people ${ }^{33}$, qualitative data from the questionnaire and interviews were analyzed using constructivist thematic analysis. We coded anonymized transcripts of the Japanese scripts in accordance with the six phases proposed by Braun and Clarke ${ }^{34}$. Initial coding was conducted by the two Japanese researchers (YM and HO). YM was the lead author, who engages in the development of PIF-rCBCC and had experienced qualitative studies relevant to SRL. HO was chosen to conduct initial coding because he is not directly engaged in the rCBCC program but had experienced qualitative studies relevant to SRL. The transcripts were thoroughly read and analyzed using an inductive coding approach until agreement on coding was achieved through repetitive face-to-face meetings between the pair.

We specifically focused on changes of SRL (motivation, learning strategies, and reflective behaviors) and PIF (individual identities as a medical professional), and attempted to explore how they think changes occurred or what they feel the changes are attributed to. Representative codes and statements were translated into English by an American professor living in Japan literate both English and Japanese (AJL). In the final phase, two other authors (JL and CV; education psychologists being familiar to SRL) joined the discussion, and a higher-level synthesis of the codes were made.

\section{Results}

\section{1) Quantitative Data}

Mean averages, standard deviations, and median averages for fifteen MSLQ-J categories plus 
gender and academic rank data at pre-program are shown in Table 4-1. No categories were significantly different between the two PIF-and original-rCBCCs groups.

The subtracted (post- minus pre-program) between-group scores in the 15 MSLQ-J categories are shown in Table 4-2. Improvement of 1. Intrinsic goal orientation and 10. Critical thinking were significantly better in PIF-rCBCCs than original rCBCCs with $\varepsilon^{2}$ values 0.096 ( $p$ $=.005)$ and $0.051(p=.041)$, respectively. In these two categories, scores at pre-program were numerically but insignificantly lower in PIF-rCBCCs than original rCBCCs.

\section{2) Qualitative Data}

In accordance with qualitative data findings, we first focused on verbatim responses implying intrinsic goal orientation or intrinsic motivation, and critical thinking. The initial coding started with responses from Q6 in the questionnaire, 'Can you describe the change in motivation for selfstudy between pre-and post-PIF rCBCC?', and from Q3 in the interview, 'Through PIF-rCBCC, is there any change in self-motivation?' in order to explore the intrinsic goal orientation or motivation among PIF-rCBCCs. Next, the initial coding proceeded with responses to Q5 in the questionnaire, 'Can you describe the change in methods and strategies you apply to self-study between pre- and post-PIF rCBCC?' and from Q4 in the interview, 'Through PIF-rCBCC, is there any change in methods and strategies you apply to self-study?' in order to explore how the PIF-rCBCC improved critical thinking. Lastly, we further coded responses from the other questions. After the initial coding agreement, we thoroughly reviewed all codes and relevant quotes and analyzed them at the broader thematic level. In the final phase, the other authors (JL and CV) joined the discussion, and a higher-level synthesis of the codes eventually resulted in three major themes corresponding to the second research question; 'How does the PIF-oriented rCBCC improve SRL?'

\section{Theme 1. Recognition of professional responsibility as the motivator for daily self-study}

Students began to link 'responsibility' entailed by future professional roles to reasons for daily self-study on diseases. They viewed in-depth communication with their mentors through the PIF-rCBCC format with PIE helpful for imagining their future professional responsibilities in a positive and objective manner.

'A lot of opening questions were, for example, what do you expect from work, what is the worst that can happen if you failed to live up to the expectations you have set for yourself, that's the situation you're working under when you're a doctor, and the first time I really felt this was the time I really should be aware of this, it was positive, and actually al though I was still just a student, I could objectively assess what I was thinking, and since I was getting actual instruction and advice from the mentor, I thought I couldn't waste this opportunity and wanted to maintain a connection to it, and because of this motivation it was really great training.' (Interview, 1D-10) 
Table 4-1. Pre-program Motivated Strategies for Learning Questionnaire-J fifteen category scores and descriptive gender and academic rank data

\begin{tabular}{|c|c|c|c|c|}
\hline & & $\begin{array}{l}\text { PIF-rCBCCs } \\
\qquad(\mathrm{N}=41)\end{array}$ & $\begin{array}{l}\text { Original rCBCCs } \\
\qquad(\mathrm{N}=41)\end{array}$ & $p$ value \\
\hline Gender & Male/Female & $30 / 11$ & $30 / 11$ & \\
\hline \multirow{2}{*}{$\begin{array}{l}\text { Academic rank } \\
\text { in the previous school year }\end{array}$} & Mean $\pm S D$ & $43.7 \pm 33.0$ & $45.3 \pm 32.4$ & \multirow{2}{*}{0.79} \\
\hline & Median & 36 & 37 & \\
\hline \multirow{2}{*}{ 1. Intrinsic goal orientation } & Mean \pm SD & $4.07 \pm 1.18$ & $4.23 \pm 1.16$ & \multirow{2}{*}{0.42} \\
\hline & Median & 4.00 & 4.25 & \\
\hline \multirow{2}{*}{ 2. Extrinsic goal orientation } & Mean \pm SD & $3.67 \pm 1.46$ & $3.69 \pm 1.44$ & \multirow{2}{*}{0.95} \\
\hline & Median & 3.75 & 3.75 & \\
\hline \multirow{2}{*}{ 3. Task value } & Mean \pm SD & $5.12 \pm 0.95$ & $4.85 \pm 1.17$ & \multirow{2}{*}{0.34} \\
\hline & Median & 5.33 & 4.83 & \\
\hline \multirow{2}{*}{ 4. Control beliefs } & Mean \pm SD & $4.92 \pm 0.92$ & $4.69 \pm 0.98$ & \multirow{2}{*}{0.09} \\
\hline & Median & 4.75 & 4.50 & \\
\hline \multirow{2}{*}{ 5. Self-efficacy } & Mean \pm SD & $3.52 \pm 1.11$ & $3.36 \pm 1.23$ & \multirow{2}{*}{0.72} \\
\hline & Median & 3.38 & 3.50 & \\
\hline \multirow{2}{*}{ 6. Test anxiety } & Mean $\pm S D$ & $3.94 \pm 1.17$ & $4.35 \pm 1.03$ & \multirow{2}{*}{0.12} \\
\hline & Median & 4.20 & 4.40 & \\
\hline \multirow{2}{*}{ 7. Rehearsal } & Mean \pm SD & $4.38 \pm 1.07$ & $4.23 \pm 0.91$ & \multirow{2}{*}{0.81} \\
\hline & Median & 4.25 & 4.25 & \\
\hline \multirow{2}{*}{ 8. Elaboration } & Mean $\pm S D$ & $4.46 \pm 1.00$ & $4.32 \pm 1.13$ & \multirow{2}{*}{0.86} \\
\hline & Median & 4.50 & 4.50 & \\
\hline \multirow{2}{*}{ 9. Organization } & Mean \pm SD & $4.45 \pm 1.35$ & $4.27 \pm 1.30$ & \multirow{2}{*}{0.66} \\
\hline & Median & 4.50 & 4.50 & \\
\hline \multirow{2}{*}{ 10. Critical thinking } & Mean \pm SD & $4.11 \pm 1.10$ & $4.30 \pm 1.21$ & \multirow{2}{*}{0.36} \\
\hline & Median & 4.20 & 4.40 & \\
\hline \multirow{2}{*}{ 11. Metacognitive regulation } & Mean \pm SD & $4.23 \pm 0.70$ & $4.18 \pm 0.82$ & \multirow{2}{*}{0.89} \\
\hline & Median & 4.25 & 4.17 & \\
\hline \multirow{2}{*}{ 12. Time and environment } & Mean $\pm S D$ & $4.63 \pm 0.85$ & $4.44 \pm 0.87$ & \multirow{2}{*}{0.38} \\
\hline & Median & 4.50 & 4.25 & \\
\hline \multirow{2}{*}{ 13. Effort management } & Mean \pm SD & $3.92 \pm 1.07$ & $3.91 \pm 0.96$ & \multirow[b]{2}{*}{0.83} \\
\hline & Median & 4.00 & 4.00 & \\
\hline
\end{tabular}


Table 4-2. Pre-post program difference Motivated Strategies for Learning Questionnaire-J fifteen category scores

\begin{tabular}{|c|c|c|c|c|c|}
\hline & & $\begin{array}{c}\text { PIF-rCBCCs } \\
\quad(\mathrm{N}=41)\end{array}$ & $\begin{array}{l}\text { Original rCBCCs } \\
\qquad(\mathrm{N}=41)\end{array}$ & $p$ value & $\varepsilon^{2}$ value \\
\hline \multirow{2}{*}{ 1. Intrinsic goal orientation } & Mean \pm SD & $0.48 \pm 1.02$ & $-0.26 \pm 1.17$ & \multirow{2}{*}{0.005} & \multirow{2}{*}{0.096} \\
\hline & Median & 0.50 & -0.25 & & \\
\hline \multirow{2}{*}{ 2. Extrinsic goal orientation } & Mean \pm SD & $0.31 \pm 1.36$ & $-0.05 \pm 1.04$ & \multirow{2}{*}{0.200} & \multirow{2}{*}{0.020} \\
\hline & Median & 0.25 & 0.00 & & \\
\hline \multirow{2}{*}{ 3. Task value } & Mean \pm SD & $0.12 \pm 1.08$ & $-0.02 \pm 1.08$ & \multirow{2}{*}{0.587} & \multirow{2}{*}{0.004} \\
\hline & Median & 0.00 & 0.00 & & \\
\hline \multirow{2}{*}{ 4. Control beliefs } & Mean \pm SD & $0.04 \pm 1.07$ & $0.02 \pm 1.16$ & \multirow{2}{*}{0.665} & \multirow{2}{*}{0.002} \\
\hline & Median & 0.00 & 0.25 & & \\
\hline \multirow[b]{2}{*}{ 5. Self-efficacy } & Mean $\pm S D$ & $0.49 \pm 1.20$ & $0.10 \pm 0.82$ & \multirow[b]{2}{*}{0.210} & \multirow[b]{2}{*}{0.019} \\
\hline & Median & 0.25 & 0.00 & & \\
\hline \multirow{2}{*}{ 6. Test anxiety } & Mean \pm SD & $0.30 \pm 1.07$ & $-0.11 \pm 1.07$ & \multirow{2}{*}{0.152} & \multirow{2}{*}{0.025} \\
\hline & Median & 0.20 & 0.00 & & \\
\hline \multirow{2}{*}{ 7. Rehearsal } & Mean \pm SD & $0.23 \pm 1.23$ & $-0.02 \pm 1.14$ & \multirow{2}{*}{0.500} & \multirow{2}{*}{0.006} \\
\hline & Median & 0.25 & 0.00 & & \\
\hline \multirow{2}{*}{ 8. Elaboration } & Mean \pm SD & $0.30 \pm 1.23$ & $0.13 \pm 1.03$ & \multirow{2}{*}{0.083} & \multirow{2}{*}{0.037} \\
\hline & Median & 0.50 & 0.00 & & \\
\hline \multirow{2}{*}{ 9. Organization } & Mean \pm SD & $0.08 \pm 1.48$ & $-0.04 \pm 1.08$ & \multirow{2}{*}{0.915} & \multirow{2}{*}{$<0.001$} \\
\hline & Median & 0.00 & 0.00 & & \\
\hline \multirow{2}{*}{ 10. Critical thinking } & Mean \pm SD & $0.48 \pm 1.08$ & $-0.06 \pm 1.21$ & \multirow{2}{*}{0.041} & \multirow{2}{*}{0.051} \\
\hline & Median & 0.60 & 0.00 & & \\
\hline \multirow{2}{*}{ 11. Metacognitive regulation } & Mean \pm SD & $0.31 \pm 0.80$ & $-0.07 \pm 0.69$ & & \\
\hline & Median & 0.16 & 0.00 & 0.000 & 0.043 \\
\hline & Mean \pm SD & $0.02 \pm 1.28$ & $0.02 \pm 1.03$ & & \\
\hline 12. Time and environment & Median & 0.00 & 0.00 & 0.700 & 0.002 \\
\hline & Mean \pm SD & $0.41 \pm 0.89$ & $0.10 \pm 0.85$ & & \\
\hline 13. Eftort management & Median & 0.25 & 0.00 & 0.092 & 0.035 \\
\hline & Mean $\pm S D$ & $0.03 \pm 1.28$ & $0.03 \pm 1.03$ & & \\
\hline 14. Peer learnıng & Median & 0.00 & 0.00 & $0.9 / 8$ & <0.001 \\
\hline & Mean \pm SD & $0.04 \pm 1.27$ & $0.04 \pm 0.84$ & & \\
\hline 15. Help seekıng & Median & 0.00 & -0.25 & 0.819 & $<0.001$ \\
\hline
\end{tabular}


At pre-program, PIF-rCBCC subjects tended to limit their learning to within the range of medical knowledge assessed by teachers of the curriculum. After the PIF-rCBCC, they became motivated to expand their area of learning beyond the subjects for testing. Expansion of learning was preparation for professional responsibility to meet social expectation and patient needs.

\footnotetext{
'Knowing what skills and knowledge the region expect of you, you can create a working image of your future situation, and it becomes motivation to learn about new areas you weren't aware of before.' (Questionnaire, 2019-19)
}

'Did my learning choices increase? Until now I was fine with studying mainly materials for the national qualifying exam, and that's best in order to get through that, but actually, yes, I'm aware that as knowledge it isn't enough. So there's also taking a look at UpToDate (medical information website), and not only using textbooks but also books about becoming a clinician.' (Interview, 1C-7)

Aside from perceived expanding area of learning subjects, students began to see self-study required for authenticity and applicability of medical knowledge in their future workplace. This was linked to Theme 3 .

'What I got was that incomplete understanding or recall wasn't going to cut it when actually applying knowledge in the clinic. I began to train with the awareness that I wouldn't be useful there if I didn't understand and remember all I learned about disease.' (Questionnaire, 2018-21)

\section{Theme 2. Strategies toward elaboration by linking their future professional task process to self-study contents}

After PIF-rCBCC, students began to imagine what they would do as unique professionals in future medical practice at a task process-based level. Because their vivid future professional image helped them identity deep responsibilities for their professional tasks, they began carefully observing mentors' complete professional tasks and began to articulate the process of the tasks. That encouraged PIF-rCPCCs to select learning strategies in which they link information in selfstudy materials to the professional task processes. Even after rCBCC, they kept learning with strategies of linking medical knowledge in self-study materials to professional tasks processes, which is referred to as 'elaboration' in MSLQ.

'After training, I began approaching study thinking about what can I do myself. It wasn't just a wide view either, since I was really thinking in detail.' (Questionnaire, 2018-8) 
'I've begun thinking about studying to prepare for when patients come, when they come for their first exam.' (Interview, 2C-11)

\section{Theme 3. Critical evaluation of learning objects to pursuit authenticity and applicability ofmedical knowledge.}

After PIF-rCBCC, students began to seek as much authentic and applicable information as possible from learning materials in their daily self-study.

'When I study a text now, I began to think how the knowledge can be used for the patient.'

(Questionnaire, 2019-13)

'It's really important to learn about disease by studying the texts and the comprehensive knowledge, but since that tertiary material is insufficient for responding to clinical questions and individual patient backgrounds, I'm not sure that knowledge is useful in clinical practice. For that, what's most meaningful are secondary materials like UpToDate, or if you still have doubts then primary material research papers. (Questionnaire, 2019-1)

After beginning to pursue authenticity and applicability of medical knowledge during their daily self-study, students found diversity and inconsistency in information from learning materials. To deal with this, they began to formulate inquiries focusing more on self-study, a variety of information resources, and different viewpoints. Through this strategic shift, critical thinking emerged in an intrinsic manner.

'I've come to think opinions are going to diverge to some extent as you get down to actually asking opinions of several different doctors, and these are choices you have to make, the stages you go through when studying.' (Interview, 1C-7)

'Having the ability to doubt, and because it's science not taking everything at face value, becoming skeptical, I think that's necessary.' (Interview, 3C-14)

'Now I try not to depend on only one research paper to know something.' (Questionnaire, 2019-10)

\section{Discussion}

To our knowledge, this is the first study investigating effects of a concrete PIF-oriented education format on SRL in undergraduate medical education. We explored whether PIF-oriented education enhances SRL by formulating two research questions. 
Regarding the first research question 'Does PIF-rCBCC improve SRL better than the original format $r C B C C$ ?', among original $\mathrm{rCBCC}$ subjects intrinsic goal orientation and some other MSLQ-J categories showed lower post-program values, while among PIF-rCBCC subjects values in most categories increased. This indicates work-based learning without PIF-oriented instruction is not effective as an educational approach for SRL with JMU medical students on short-term rural placement. On the other hand, significant improvement in intrinsic goal orientation and critical thinking in PIF-rCBCCs compared with original rCBCCs implies PIF education in future work settings may promote SRL.

The original format $\mathrm{rCBCC}$ had already fulfilled its first goal of training in future work settings with future role models. Therefore, the quantitative comparative approach in this study by design evaluated effects of the additional SRL-based components. However, we do not mean to demean importance of the first goal. For instance, we believe students would not be able to recognize their future professional responsibility nor be as intrinsically motivated solely from interactions with university-based instructors, as previous studies suggest ${ }^{16,17,35}$.

The qualitative analysis supports MSLQ-J results. Pertaining to intrinsic goal orientation, the thematic analysis showed students' recognition of their future professional 'responsibility' was key to fostering it. Also, recognition of professional responsibility led to improvement of critical thinking or critical evaluation of learning materials in order to pursue authenticity and applicability of information for professional task processes. The consistency between quantitative and qualitative data was notable for understanding outcomes of the PIF-oriented education on SRL, insofar similar results demonstrate construct validity for additional education in the rCBCC program.

There was no statistical significance in the difference between PIF- and original rCBCC regarding elaboration in MSLQ-J data. However, qualitative analysis illuminated that PIFrCBCCs' attention to professional task processes triggered a strategic shift from memorization to elaboration of knowledge in accordance with their future professional task processes. Reasons accounting for the discrepancy could be the number of participants and sensitivity of outcome measurement. Further research is needed to address these issues. However, discrepancies between qualitative and quantitative evaluation does not indicate a study failure ${ }^{36}$. By incorporating both quantitative and qualitative data from a constructivist paradigm ${ }^{33,36}$, we thought that this study meaningfully captured educational benefits of shifting toward more PIF-oriented strategies.

The link between PIF and motivational aspects of SRL could be explained by 'identity-based motivation' proposed by Oyserman ${ }^{37}$ as 'the readiness to engage in identity-congruent action and to use identity-congruent mindsets in making sense of the world.' According to her theory, people are more likely to interpret difficult experiences by implying task importance when an accessible identity feels congruent with the task ${ }^{38}$. In the context of this study, the increasing professional identity as a 'physician-to-be' strengthens the perception of importance of engaging in challenges at the practice site and self-regulate their learning behaviors by using identitycongruent mindsets and making sense of future learning tasks. Therefore, theoretically SRL in 
medical education should be linked more to identity formation of professional medical practitioners.

Since the current study tested the PIF-oriented education format in only one school year curriculum of one medical university, general applicability remains unclear. If introduced to other institutions, we suggest program directors first ask students to articulate their future images and norms of professionalism, then carefully select mentors in accordance with students' statements. This PIF-oriented education format helped students imagine their futures and observe professional behaviors of JMU alumni mentors in clinical clerkships. Accordingly, students paid more attention to learning behaviors of their mentors. Therefore, teachers should recognize they are models not only for professionalism but also for self-regulated and life-long learners. To improve PIF-oriented SRL education quality, faculty development is essential ${ }^{12,13}$.

\section{Strengths and limitations}

The strength of this study is subjects were Japanese students considered to engage in less selfregulation than Western counterparts ${ }^{10,11,20}$. We believe our study can provide educators with evidence PIF-oriented educational schemes promote better learning behaviors in institutions struggling to promote undergraduates' SRL.

Another strength is there were few formal classes or training programs - i.e., intervening confounders -- except for PIF-rCBCC or the original format $\mathrm{rCBCC}$ between pre and post data collection (Figure 4-2). We believed the study schedule without other educational interventions optimized learning comparison outcomes between the two groups.

This study has some limitations. First, we did not measure students' PIF levels before and after the PIF-oriented education. Therefore, we cannot fully attribute higher intrinsic goal orientation and critical thinking in quantitative data to better professional identity levels. However, qualitative data supported their medical practitioner professional identity formed with strong perceptions of 'responsibility'. These results suggest construct validity of the educational experience.

Second, we did not investigate alumni mentor perceptions regarding this PIF-oriented education format and Google Forms usability. According to comments, both students and doctors enjoyed opportunities for interactive communication. However, future studies need focus on mentor perceptions to ensure significance of the PIF-oriented education format.

Third, we have not followed long-term outcomes of PIF or SRL regarding self-study and clinical outcomes. According to previous studies ${ }^{16,38}$, identity is flexibly attuned to immediate situations rather than fixed in memory. Plus, frequently and fluently cued identities form into stable ones. Therefore, we predict repetitive PIF-oriented education could strengthen learners' SRL. 


\section{Conclusions}

Allowing for these limitations and need for further research, this study indicated future work setting-based PIF-oriented undergraduate clinical clerkships with alumni mentors increased immediate intrinsic goal orientation and promoted shift to SRL. Their SRL was characterized as task process-based elaboration, with critical thinking emerging from pursuit of authenticity and knowledge applicability in medical practice.

\section{List of abbreviations}

SRL: Self-regulated learning; PIF: Professional identity formation; MSLQ: Motivated Strategies for Learning Questionnaire; rCBCC: Rural community-based clinical clerkship; PIE: Professional identity essay

\section{References}

1. Frank JR. The CanMED 2005 Physician Competency Framework: Better Standards, Better Physicians, Better Care. Ottawa, Canada: Royal College of Physicians and Surgeons of Canada; 2005.

2. Sandars J, Cleary TJ. Self-regulation theory: application to medical education: AMEE Guide No 58. Med Teach. 2011; 33: 875-86.

3. Brydges R, Butler D. A reflective analysis of medical education research on self-regulation in learning and practice. Med Educ. 2012; 46: 71-9.

4. Berkhout JJ, Helmich E, Teunissen PW, Van der Vleuten CP, Jaarsma ADC. Context matters when striving to promote active and lifelong learning in medical education. Med Educ. 2018; 52: 34-44.

5. Zimmerman BJ. A social cognitive view of self-regulated academic learning. J Educ Psychol. 1989; 81: 329-39.

6. Artino AR, Jones KD. AM Last Page: Self-regulated learning—a dynamic, cyclical perspective. Acad Med. 2013; 88: 1048.

7. Zimmerman BJ: Attaining self-regulation: A social-cognitive perspective. In Handbook of SelfRegulation. Edited by: Boekaerts M, Pintrich P, Ziedner M. San Diego, CA, USA: Academic Press; 2000: 13-39.

8. Rich JV. Proposing a model of co-regulated learning for graduate medical education. Acad Med. 2017; 92: 1100-4.

9. Van Houten-Schat MA, Berkhout JJ, Van Dijk N, Endedijk MD, Jaarsma ADC, Diemers AD. Self-regulated learning in the clinical context: a systematic review. Med Educ. 2018; 52: 1008-15

10. Matsuyama Y, Nakaya M, Okazaki H, Leppink J, Van der Vleuten C. Contextual attributes promote or hinder self-regulated learning: A qualitative study contrasting rural physicians with undergraduate learners in Japan. Med Teach. 2018; 40: 285-95.

11. Matsuyama Y, Nakaya M, Okazaki H, Lebowitz AJ, Leppink J, van der Vleuten CPM. Does changing from a teacher-centered to a learner-centered context promote self-regulated learning: a qualitative study in Japanese undergraduate setting. BMC Med Educ. 2019; 19: 152. 
12. Cruess RL, Cruess SR, Boudreau JD, Snell L, Steinert Y. Reframing medical education to support the development of a professional identity. Acad Med. 2014; 89: 1446-51.

13. Ziring D, Dnoff D, Grosseman S, Langer D, Esposito A, Jan MK, Rosenzweig S, Novack D. How do medical schools identify and remediate professionalism lapses in medical students? A study of U.S. and Canadian medical schools. Acad Med. 2015; 90: 913-20.

14. de Lasson L, Just E, Stegeager N, Malling B. Professional identity formation in the transition from medical school to working life: a qualitative study of group-coaching courses for junior doctors. BMC Med Educ. 2016; 16: 165.

15. Barnhoorn PC, Houtlosser M, Ottenhoff-de Jonge MW, Essers GTJM, Numans ME, Kramer AWM. A practical framework for remediating unprofessional behavior and for developing professionalism competencies and a professional identity. Med Teach. 2019; 41: 303-308.

16. Goldie J. The formation of professional identity in medical students: considerations for educators. Med Teach. 2012; 34: e641-8.

17. Baernstein A, Oelschlager AM, Chang TA, Wenrich MD. Learning professionalism: perspectives of preclinical medical students. Acad Med. 2009; 84: 574-81.

18. Matsumoto M, Kajii E. Medical education program with obligatory rural service: analysis of factors associated with obligation compliance. Health Policy. 2009; 90: 125-132.

19. Okayama M, Kajii E. Does community-based education increase students' motivation to practice community health care?--a cross sectional study. BMC Med Educ. 2011; 11: 19.

20. Matsuyama Y, Muijtjens AM, Kikukawa M, Stalmeijer R, Murakami R, Ishikawa S, Okazaki H. A first report of East Asian students' perception of progress testing: a focus group study. BMC Med Educ 2016; 16: 245.

21. Kalet A, Buckvar-Keltz L, Harnik V, Monson V, Hubbard S, Crowe R, Hyuksoon SS, Yingling S. Measuring professional identity formation early in medical school. Med Teach. 2017; 39: 255-261.

22. Kegan R. In over our heads: The mental demands of modern life. Cambridge, MA, USA: Harvard University Press; 1994.

23. Bidjerano T. Gender Differences in Self-Regulated Learning. Paper presented at the Annual Meeting of the Northeastern Educational Research Association (36th, Kerhonkson, NY, Oct 19-21, 2005)

24. Ray MW, Garavalia LS, and Gredler ME. Gender Differences in Self-regulated Learning, Task Value, and Achievement in Developmental College Students. Paper presented at the Annual Meeting of the American Educational Research Association (Chicago, IL, April 21-25,2003)

25. Lucieer SM, Jonker L, Visscher C, Rikers RM, \& Themmen AP. Self-regulated learning and academic performance in medical education. Med Teach. 2016; 38: 585-593.

26. Creswell J. Mixed methods designs. In Educational Research. 4th ed. Edited by: Creswell JW. Boston, USA: Pearson; 2012: 564-605.

27. Pintrich PR, Smith D, Garcia T, McKeachie WJ. A manual for the use of the Motivated Strategies for Learning Questionnaire (Technical Report 91-B-004). Michigan, USA: The Regents of the University of Michigan; 1991.

28. Soemantri D, Mccoll G, Dodds A. Measuring medical students' reflection on their learning: modification and validation of the motivated strategies for learning questionnaire (MSLQ). BMC Med Educ. 2018; 18: 274.

29. Cho KK, Marjadi B, Langendyk V, Hu W. Medical student changes in self-regulated learning during the transition to the clinical environment. BMC Med Educ. 2017; 17: 59.

30. Kim KJ, Jang HW. Changes in medical students' motivation and self-regulated learning: a preliminary study. Int J Med Educ. 2015; 6: 213-215. 
31. Hennink MM, Kaiser BN, Marconi VC. Code saturation versus meaning saturation: How many interviews are enough? Qual Health Res. 2016; 27: 591-608.

32. Jamovi project. Jamovi version 1.0.7.0. 2019. Retrieved from: https://www.jamovi.org/ (Accessed: 5 December 2019).

33. Bergman E, de Feijter J, Frambach J, Godefrooij M, Slootweg I, Stalmeijer R, van der Zwet J. AM last page: A guide to research paradigms relevant to medical education. Acad Med. $2012 ; 87: 545$.

34. Braun V, Clarke V. Using thematic analysis in psychology. Qual Res Psychol. 2006; 3: 77-101.

35. Stockley AJ, Forbes K. Medical professionalism in the formal curriculum: 5 th year medical students' experience. BMC Med Educ. 2014; 14: 259.

36. Tonkin-Crine S, Anthierens S, Hood K, Yardley L, Cals JW, Francis NA, Coenen S, van der Velden AW, Godycki-Cwirko M, Llor C, Butler CC, Verheij TJ, Goossens H, Little P; GRACE INTRO/ CHAMP consortium. Discrepancies between qualitative and quantitative evaluation of randomised controlled trial results: achieving clarity through mixed methods triangulation. Implement Sci. 2016; 11: 66 .

37. Oyserman D. Identity-based motivation: Implications for action-readiness, procedural-readiness, and consumer behavior. J Consum Psychol. 2009; 19: 250-60.

38. Oyserman D., Lewis Jr NA, Yan VX, Fisher O, O’Donnell SC, Horowitz E. An identity-based motivation framework for self-regulation. Psychol Inq. 2017; 28: 139-147. 
Supplemental Data 4-1. Google Forms for Professional Identity Formation-oriented rural community-based clinical clerkship

PART1: Professional Identity Essay (Quoted from Kalet A, et al. Med Teach 2017;39(3):255-261)

Directions: This essay explores how you understand the meaning of professionalism at this point in your development and how that relates to the formation of an ethical professional identity. Research suggests that the meaning of professionalism and one's identity with the profession evolves throughout one's career. Respond as fully as you can to each of the questions.

The purpose is to engage you in self-assessment, reflection and goal setting.

Please answer these questions as fully as you can in 1 hour. Write at least a paragraph for each question.

Q1. What does being a member of the medical profession mean to you? How did you come to this understanding?

Q2. What do you expect of yourself as you work towards becoming a full-fledged physician?

Q3. What will the profession expect of you?

Q4. What conflicts do you experience or expect to experience between your responsibility to yourself and others-patients, family, and profession? How do you resolve them?

Q5. What would be the worst thing for you if you failed to live up to the expectations you have set for yourself?

Q6. What would be the worst thing for you if you failed to live up to the expectations of your patients?

Q7. What would be the worst thing for you if you failed to live up to what society expects of physicians? How did you come to this understanding?

Q8. Think of a physician you consider an exemplar of professionalism. Describe why you chose this person, illustrating with an incident or pattern of decisions or actions that supports your choice.

Q9. Reflect on your experiences in medical school or in the community that have been critical in fostering change in your understanding of what it means to be a professionalto be a physician. 
Continued Supplemental Data 4-1. Google Forms for Professional Identity Formation-oriented rural community-based clinical clerkship

PART 2. (Pre-rCBCC) Please fulfill Q1-5 if you are a medical student, or fulfill Q6-8 if you are an attending graduate.

Question 1 (for students): Please describe your future self-image as a doctor at this moment.

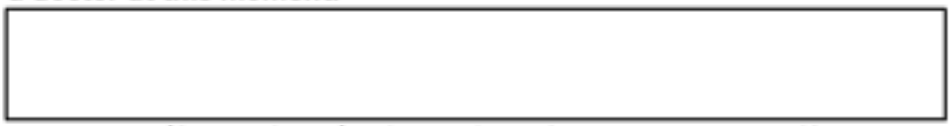

Question 2 (for students): Please describe your present self-image as a medical student at this moment.

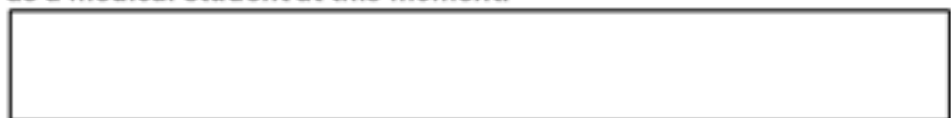

Question 3 (for students): To tell your mentor about yourself, please write confidentially about knowledge, skills and competencies you think you have already acquired in past clinical clerkships.

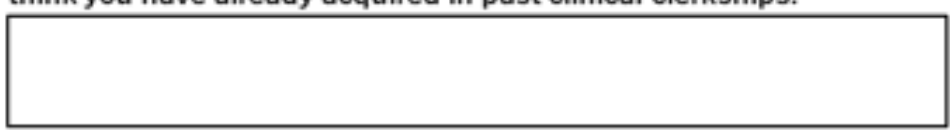

Question 4 (for students): To tell your mentor about yourself, please write about knowledge you want to learn and skills you want to experience during the clinical clerkship.

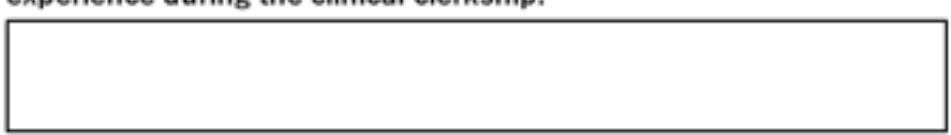

Question 5 (for students): Please be honest with yourself and write what you must learn during the clinical clerkship.

Question 6 (for graduates): Please describe your present self-image as a doctor at this moment.

Question 7 (for graduates): Please write what students can learn during their stay after reading comments from students on Q3 and 4.

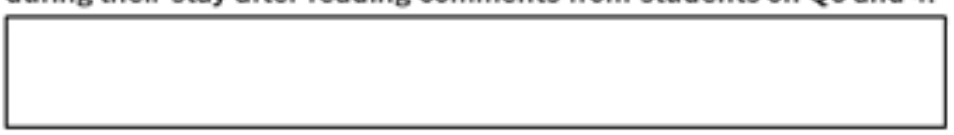

Question 8 (for graduates): Please write what you want students to complete after reading comments from students on Q5. 
Continued Supplemental Data 4-1. Google Forms for Professional Identity Formation-oriented rural community-based clinical clerkship

PART 2. (Post-rCBCC) Please fulfill Q1-5 if you are a medical student, or fulfill Q6-8 if you are an alumni mentor.

Question 1 (for students): Please describe your future self-image as a doctor at this moment.

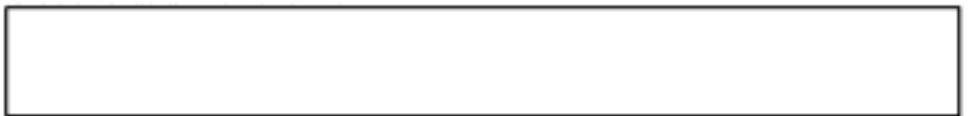

Question 2 (for students): Please describe your present self-image as a medical student at this moment.

Question 3 (for students): To tell your alumni about yourself, please write confidentially about knowledge, skills and competencies that you think you have already acquired in past clinical clerkships.

Question 4 (for students): To tell your alumni about yourself, please write about knowledge you want to learn and skills you want to experience during the clinical clerkship.

Question 5 (for students): Please be honest with yourself and write what you must learn during the clinical clerkship.

Question 6 (for graduates): Please describe your present self-image as a doctor at this moment.

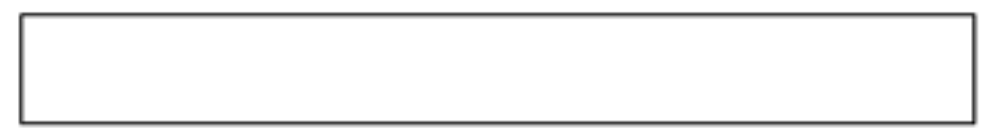

Question 7 (for graduates): Please write what students can learn during their stay after reading comments from students on Q3 and 4.

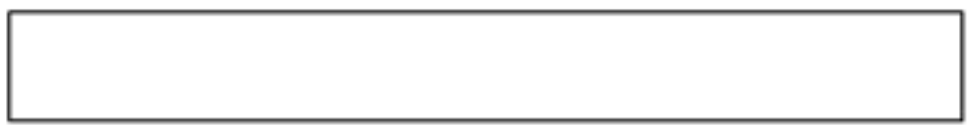

Question 8 (for graduates): Please write what you want students to complete after reading comments from students on Q5. 
Supplemental Data 4-2. Guidebook for teacher in Professional Identity Formation-oriented rural community-based clinical clerkship

\section{$<$ Guidebookfor teachers in PIF-oriented rCBCCfor SRL >}

$\square$ Self-regulated learning SRL is a competency describing how actively learners participate in their own learning process from metacognitive, motivational, and behavioral perspectives without over-dependence on teacher instruction.

$\square$ This competency is important for life-long learning to improve professional knowledge and skills as community medical practitioners.

$\square$ This rural community-based clinical clerkship ( $\mathrm{rCBCC}$ ) is characterized by (1) future GPs experiencing medical practice in their future workplaces, and (2) medical practice experience with alumni mentors (graduates working as rural physicians).

$\square$ A new learning program in this clinical clerkship is being introduced in order to make most use of these characteristics. The program 'PIF-oriented $\mathrm{rCBCC}$ ' is named after our hypothesis that helping students' professional identity formation (PIF) during rCBCC may foster SRL. The aim of the program is to help students' awareness of their professional identity as a rural physician, promote their attention and admiration to future role models, and accordingly foster students' SRL.

\section{We would like you to do three things as follows.}

(1) Between July (pre-rCBCC) and September (post-rCBCC), please see your students as immature but diligent professionals and have honest weekly communication with them in a respectful manner.

(2) Through Google Forms, please understand how they perceive their professional roles and what they want to study during $\mathrm{rCBCC}$, and explain what professional experience you want them to have as future rural GPs. Before the start of rCBCC, please make and revise concrete learning plans for $\mathrm{rCBCC}$ in accordance with sufficient talks between you and your students.

(3) During rCBCC, please provide practical roles for students, motivate them to complete the role's tasks and give productive feedback on their attitude and behavior, and show how to apply your self-study outcomes to your professional role.

\section{Please check the following checkbox to complete instruction for PIF-oriented rCBCC. 1. Pre-rCBCC period (July to August)}

$\square$ In early July, you will receive an e-mail with the link to Google Forms from your students in addition to documents you usually receive for the original rCBCC. The Google Forms will display the student's short essay about professionalism (professional identity essay invented by Kalet A, et al. Med Teach 2017), their future image, the gap between their 
current and future selves, and expectations about learning in rCBCC (see also Supplement Data 4-1).

$\square$ Please read your students' essays and make comments on them in Google Forms. Additionally, please fill in the Google Forms questionnaire for teachers regarding your current professional roles, the value and norms as medical professionals, and what you want students to learn during rCBCC.

$\square$ Please have continuous communication with your students in a respectful manner as if you see them as immature but admirable and diligent medical professionals. Aside from Google Forms, you are allowed to have communication with them by email and phone as necessary.

$\square$ Over the placement, you and your students will be able to clarify what students can learn and what kind of experience is significant to foster their professionalism and SRL. Please make a concrete plan in which students are given practical medical roles and supposed to complete tasks without full instruction.

\section{During rCBCC}

$\square$ Basically, you are allowed to attend your students as you did in the original rCBCC in the past.

Additionally, please let students experience learning in accordance with the concrete plan made in pre-rCBCC period.

$\square \quad$ In this extra-learning component, students will be given certain roles in medical practice to study by themselves to complete the role's tasks. Please give them feedback in a respectful manner to motivate them to do self-reflection. Of course you can stop them if you see unprofessional or harmful behaviors.

$\square$ In addition to the extra-learning, we hope you demonstrate your own self-study. We believe that students pay more attention to SRL strategies when they see their mentors conduct selfstudy to solve medical problems in students' future work settings. We hope you show your self-study with regard to the four following aspects. (1) What kind of processes are there in your self-study? (2) What kind of strategies and tools are used in your self-study? (3) How do you motivate yourself? (4) How do you monitor and evaluate your learning outcomes? The aim of your self-study demonstration is 'to encourage students to refrain from rote-learning or test-oriented monotonous learning.' It would be nice if you study a disease your student has encountered during the rCBCC. 


\section{Post-rCBCC period (in September)}

Y You will receive an e-mail with the link to Google Forms from your students again. The Google Forms will show students' short essay about professionalism (professional identity essay), their future image, the gap between their current and future selves, and their experiences of learning in $\mathrm{rCBCC}$.

$\square$ Please read your students' essays and make comments on them in Google Forms. Additionally, please fill in the questionnaire in Google Forms for teachers regarding your current professional roles, the value and norms as medical professionals, and what you want students to learn further.

- Please have continuous communication with your students in a respectful manner as if you see them as immature but admirable and diligent medical professionals. Aside from Google Forms, you are allowed to have communication with them by email and phone as necessary.

Thank you for your cooperation for the PIF-rCBCC. If you have any questions, please feel free to contact us.

Address:

Telephone:

E-mail: 
Supplemental Data 4-3. Questionnaire and interview form for investigating students' daily self-study activities

\section{$<$ Questionnaire $>$}

Please report your latest experience of self-study about an unfamiliar disease after PIF-rCBCC and describe how you studied by answering following the questions.

What kind of disease did you self-study?

Q1. Can you recollect your experience on the date of studying about the unfamiliar disease (from definition to clinical features, diagnostic procedure, treatment, and prognosis) and describe when, where, and how you studied this?

Q2. What methods and strategies did you apply to self-study when studying the disease?

Q3. How did you motivate yourself when studying the disease?

Q4. How did you monitor and evaluate the outcome of self-study?

Q5. Can you describe the change in methods and strategies you apply to self-study between pre-and post-PIFrCBCC?

Q6. Can you describe the change in motivation for self-study between pre-and post-PIF $r C B C C$ ?

Q7. Can you describe the change in methods and strategies you use to monitor and evaluate outcomes of self-study between pre-and post-PIF $\mathrm{rCBCC}$ ?

\section{$<$ Interview form >}

$\checkmark$ Please show the following documents to each interviewee while saying 'Please read these documents before the interview.'

(1) Interviewee's Google Forms at pre-PIF rCBCC (see also Supplemental Data 4-1.)

(2) Interviewee's Google Forms at post-PIF rCBCC (see also Supplemental Data 4-1.)

(3) Interviewee's answers in the questionnaire

$\checkmark$ Let interviewees read these documents for 5 minutes before the interviews. 
Q1. In Google Forms for PIF-rCBCC you were asked to note your future 'self' as a physician. Is there any change in your future self-image after PIF-rCBCC?

Q2. After PIF-rCBCC, is there any change in your self-study behaviors?

Q3. Through PIF-rCBCC, is there any change in self-motivation?

Q4. Through PIF-rCBCC, is there any change in methods and strategies you apply to self-study?

Q5. Through PIF-rCBCC, is there any change in methods and strategies for self-reflection?

(Please take enough time for each question. This is a semi-structured interview to explore deeper insights of interviewees. Therefore, you are allowed to make questions by referring to information written in the three documents or interviewees'statements in Q1-5.) 

Chapter 5

Limited effects from professional identity formation-oriented education on selfregulated learning in a hybrid problembased learning curriculum: a mixedmethod study in Japan 


\section{Abstract}

Background: Developing self-regulated learning (SRL) in a teacher-centered curriculum is challenging. Previous studies indicate professional identity formation (PIF), or the formation of self-identity with internalized values and norms of professionalism, might improve SRL We designed a PIF-oriented education format for hybrid problem-based learning (PBL) and tested its effectiveness on SRL in a mixed research method.

Methods: A randomized controlled crossover trial was conducted using 112 pre-clinical year students at Jichi Medical University. In the six one-day PBL sessions, Groups A $(n=56$, female 18 , mean age $21.5 \mathrm{y} \pm 0.7)$ and $\mathrm{B}(n=56$, female 11 , mean age $21.7 \mathrm{y} \pm 1.0)$ experienced PIForiented pre-PBL learning: Group A had three sessions in the first half, B in the second half. Between-group PIF and SRL levels were compared using Professional Identity Essay (PIE) and Motivated Strategies for Learning Questionnaire (MSLQ). A qualitative thematic analysis of potential reasons for PIF improvement was performed using the PIE description.

Results: Two-level regression analyses showed moderate improvement of PIF stages over time $\left(R^{2}=0.069\right)$, regardless of intervention. PIF-oriented pre-PBL learning alone did not significantly improve PIE stages or MSLQ scores. Thematic analysis indicated that PIF-oriented pre-PBL learning helped students recognize realistic difficulties in clinical practice, and articulate professional image and values. However, encounters in extracurricular clinical settings had diverse and meaningful impacts on PIF.

Conclusions: Limited effect of PIF-oriented pre-PBL learning on PIF and SRL indicate challenges in SRL-oriented education for pre-clinical year students within a teacher-centered hybrid PBL curriculum.

Keywords: Self-regulated learning, Professional identity formation, Problem-based learning, Teacher-centered learning, Learning management system. 


\section{Background}

Medical professionals need to update knowledge autonomously in line with rapid advances in medicine. Therefore, life-long learning self-regulation has become an essential competency for medical professionals ${ }^{1-4}$. Self-regulated learning (SRL) is defined as learners' active participation in learning process from metacognitive, motivational, and behavioral perspectives ${ }^{5}$. Zimmerman described SRL as a cyclical process in three phases ${ }^{6,7}$.

1) Forethought phase: Learners set goals and choose strategies to attain them

2) Performance phase: Learners monitor and control behavior to attain goals

3) Self-reflection phase: Learners formulate new goals and strategies for future situations

Because pre-clinical education cannot prepare learners for all challenges faced in less-structured learning situations in clinical clerkships, the robust development of SRL-oriented education in pre-clinical settings is justified ${ }^{8-10}$. Actually, lack of readiness to engage in SRL may result from the pre-clinical curriculum.

\section{SRL in problem-based learning}

Problem-based learning (PBL) is defined as learning from processes of working towards understanding or resolution of a problem ${ }^{11}$. Essential goals of PBL include supporting knowledge structuring in clinical contexts, developing clinical reasoning skills and self-directed learning skills, and increasing students' learning motivation ${ }^{12}$.

Theoretically, students acquire SRL skills through repeated PBL sessions. For instance, students extract problems in a self-reflective manner (corresponding to the self-reflection phase) and formulate learning goals in PBL discussion sessions (corresponding to the forethought phase). Additionally, participants undertake self-study between PBL discussion sessions to accumulate relevant information, so discussion sessions are more meaningful (corresponding to the performance phase) $)^{2,6,12-13}$.

While PBL has been recognized in medical education around the world, in some regions, its operation has detracted from the original model due to educational culture and resource issues ${ }^{14}$. In Japan, PBL sessions are held only several times a year in a lecture-based curriculum, and limited SRL is promoted. In such a 'hybrid' PBL curriculum ${ }^{14}$, attempts have been made to promote SRL somehow ${ }^{15-17}$. This study describes a new approach with similar goals in a Japanese setting.

\section{Professional identity formation as a promoting factor of SRL}

Previous studies indicate individual future image as a unique professional could promote clinical student readiness to accept diverse learning strategies in a self-regulated manner ${ }^{18,19}$. Therefore, 
professional identity formation (PIF) could be a critical factor in promoting SRL in undergraduate clinical settings.

PIF is defined as formation of "a representation of self, achieved in stages over time during which the characteristics, values, and norms of the medical profession are internalized ${ }^{20}$." Cruess et al. advocate that PIF results in individuals considering their clinical environment behavior ${ }^{20}$. However, while many studies have explored outcomes of PIF-oriented medical education regarding professional and unprofessional behaviors ${ }^{21-26}$, only Matsuyama et al. ${ }^{18,19}$ have directly assessed effects of PIF-oriented educational approaches on learning behaviors. One study ${ }^{19}$ illuminated a PIF-oriented education format with a functional communication platform between students and their physician mentors. The format provided in-depth communication opportunities regarding students' future self-images, plus norms and values of professionalism. Eventually, PIF-oriented education promoted intrinsic motivation and strategic improvement with elaboration and critical thinking ${ }^{19}$.

\section{PIF-oriented learning format for PBL}

In this study, a PIF-oriented education format based on Matsuyama et al.'s design was arranged for PBL to improve SRL in a university adopting a hybrid PBL model due to teacher-centered culture $^{14,17-18}$.

Our format is characterized by pre-PBL in-depth communication

1) regarding current values or norms of professionalism and the formation of students' future medical professional images;

2) linking students' self-image as a medical professional to PBL scenario settings; and

3) receiving tips for learning strategies in PBL based on 1) and 2) from their future role models.

In this study, we tested the effects of PBL with PIF-oriented pre-PBL learning (PIF-oriented $\mathrm{PBL}$ ) on SRL in pre-clinical year students by a mixed-method explanatory approach.

The primary purpose of this study is to verify our hypothesis that the formation of individual professional identity via the PBL model could improve SRL even in hybrid PBL. If PIF or SRL improved in this context, the secondary purpose is to understand how. To clarify the purposes of the study, we formulated four research questions (RQ1-4).

RQ1. Does PIF-oriented PBL improve PIF levels in a hybrid PBL curriculum?

RQ2. Does PIF-oriented PBL improve SRL levels in a hybrid PBL curriculum?

RQ3. Do SRL and PIF levels correlate with each other?

RQ4. If PIF or SRL improves in this study, why? 
This study was approved by the university's ethics committee (reference number: 18-168). Informed consent was obtained from all participants. This study was conducted from May 2019 to April 2020.

\section{Methods}

\section{Settings}

Jichi Medical University (JMU) is a private medical university founded in 1972 in Japan. The curriculum of JMU complies with the standardized model core curriculum outlining fundamental learning contents for undergraduate medical education in Japan ${ }^{27}$. While the preclinical curriculum is partly integrated, it remains mostly stepwise: Before Year 4 to 6 (Y4-6) clinical clerkship, students mainly learn clinical medicine in traditional didactic lectures, and their progress is assessed through end-of-unit tests. JMU students study liberal arts in the first and second trimesters of Y1. Lectures and experiments in basic medicine also begin in Y1 second trimester. Clinical medicine lectures start from Y2 second trimester. Each basic and clinical medicine class is capped by end-of-unit tests where lecturers create test items based on lecture content. Before $\mathrm{Y} 3$ end, students finish almost all subject lectures in basic and clinical medicine.

Y3 has seven sessions of one-day hybrid PBL, each divided into four segments: morning case discussion for the formulation of self-study objectives, self-study period for research on objectives and preparation for afternoon discussion, afternoon discussion including within-group information sharing, and a 60-minute wrap-up lecture from a specialist. In every PBL, faculty facilitators assess each student's opinion statements, cooperative attitudes, and appropriateness of self-study using a 3-point scale. However, the assessment does not affect grade point average, and students never receive individual feedback.

Currently, students read the introductory part of a clinical scenario before PBL and preview morning discussion content. For example:

\section{'A 56-year-old man came to your hospital because he had 20-min anterior chest oppression after breakfast this morning. Please find clinical problems or possible differential diagnoses as best you can.'}

The full story containing clinical history and findings is provided on the date of the PBL session.

Because of prevailing teacher-centered education culture at this university, implementing large-scale curriculum reform towards full PBL-based implementation is difficult. Students attend only seven one-day hybrid PBL sessions a year with the rest of the Y3 curriculum lecturebased. This partial use of the PBL concept does not guarantee its full efficacy ${ }^{14}$. 


\section{PIF-oriented PBL}

The core component of the PIF-oriented PBL is the pre-PBL communication platform composed of an online instruction video and the essay format. The instruction video aimed at encouraging students to articulate their future image as an independent medical professional tackling patient problems via life-long learning. The essay format meant to provide pre-PBL in-depth communication between students and their future role models (Figure 5-1).

After watching the instruction video and reading the PBL scenario introductory, students were asked to answer three essay questions:

Q1: Please recall as much knowledge as possible you have about this case.

Q2: Please formulate your future professional images, and articulate how useful this PBL case-study would be to you as a doctor responsible for this case.

Q3. Based on your answer for Q2, please articulate how you will optimize your self-study for this case to make this opportunity most meaningful.

Aside from these questions, students were asked to submit the professional identity essay (PIE) proposed by Kalet et al. ${ }^{28}$ three times. PIE is useful for helping learners articulate their values and norms about medical professionalism, and teachers provide feedback by referring to rubrics based on Kegan's constructive developmental theory ${ }^{28,29}$. This study used a Japanese version of PIE (PIE-J) as a reference for mentors' feedback on Q1-3 and as an assessment tool for students' PIF levels (see also Instruments).

Figure 5-1. Conceptual framework of PIF-oriented PBL with pre-PBL learning

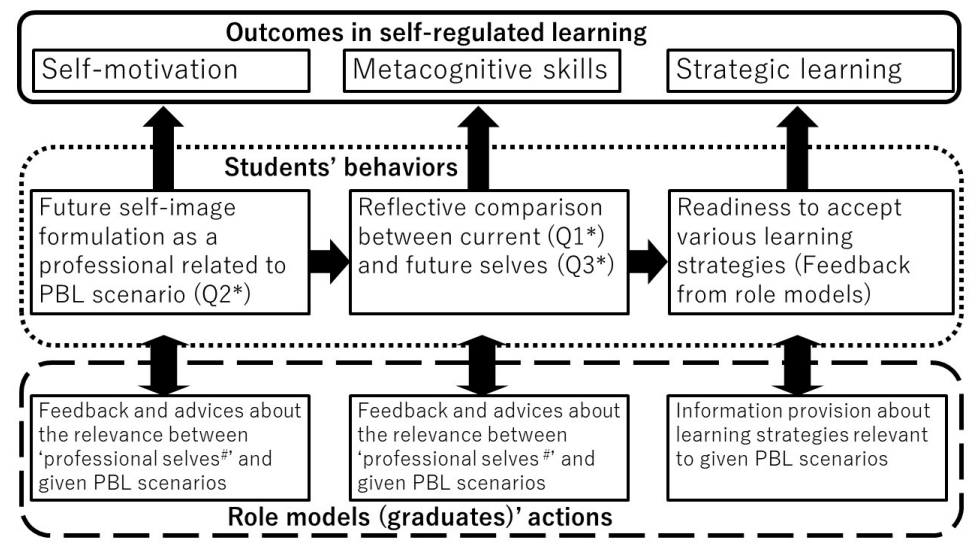

${ }^{*}$ Q1-3 mean the question item number in Moodle pre-PBL learning form.

\# 'professional selves' means not only students' self-images illustrated in Q2 and Q3 but also students' values and norms illustrated in Professional Identity Essay. 
Those materials were provided on a Moodle online learning management system. Through the Moodle platform, eligible JMU-graduate mentors provided feedback on Q1 to Q3 by simultaneously referring to each student's PIE. As a rule, the mentor feedback did not contain hints for the PBL scenario in order to avoid teacher-centered instruction. In this study, six JMU graduates with clinical experience of 18-37 years were chosen as feedback providers. All of them have agreed to received intensive training for appropriate PIE use and feedback on Q1-3 before the study.

We hypothesized Moodle-based pre-PBL learning would raise student awareness of their future professional image and the relevance of PBL scenarios for their future professional selves. We also proposed that a clearer image of their future professional selves and relevance of PBL contents would encourage them to apply learning strategies encouraged by thoughtful mentor comments. Overall, we expected that the pre-PBL PIF-oriented education format would promote PIF and accordingly improve SRL.

\section{Participants and design}

We used a quantitative analysis for RQ1 to RQ3 and a mixed explanatory method to address RQ4 regarding causes of PIF or SRL improvement. We used this method because we expected qualitative analysis would illuminate the mechanisms for PIF or SRL changes in this research context; i.e., qualitative data could help explain quantitative results ${ }^{30}$.

\section{1) Quantitative Approach: RQ1-RQ3}

A randomized controlled crossover trial was designed for the quantitative arm. All JMU 2019 Y3 students $(n=124)$ were invited to participate in this research. Eventually, 112 agreed were randomly divided into two groups: Group $\mathrm{A}(n=56$, female $=18$, male $=38$, mean age $21.5 \mathrm{y} \pm 0.7)$ and Group B $(n=56$, female $=11$, male $=45$, mean age 21.7y \pm 1.0$)$. Group A used Moodle-based PIF-oriented format before the second to fourth PBL, while group B did before the fifth to seventh PBLs in 2019. Both groups conducted the six one-day PBLs in the same manner on PBL dates, and SRL and PIF levels were compared between the two groups (Figure 5-2). Group A and B did not mix in the PBL group session. We hypothesized that PIF and SRL levels should improve in parallel, i.e., Group A in the first half of the research period, with Group B becoming equivalent to Group A in the second half. 
Figure 5-2. Study design in the quantitative part

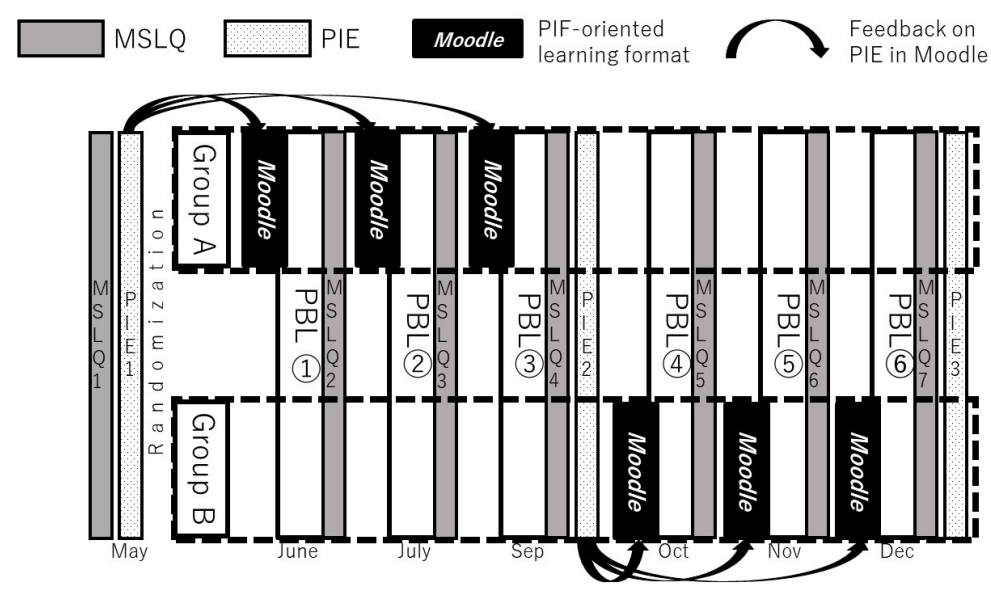

\section{2) Qualitative Approach: $R Q 4$}

Following quantitative analysis results, we explored potential reasons for PIF improvement using responses from the PIE-J from 112 students (see also Instruments). The analysis was conducted in March and April of 2020.

\section{Instruments}

\section{1) PIF data collection}

For quantitative analysis, the PIF levels for norms and values of professionalism were measured using PIE, an essay-based measurement tool with 9 question items. Referring to Dr. Kalet and colleagues' rubric $^{28}$ based on Kegan's identity stage ${ }^{28,29}$, assessors chose learners' professional identity levels from Stage II to II/III, III, III/IV, IV, IV/V, and V. The PIF measurement by PIE has been validated in undergraduate setting ${ }^{28,31}$. In this study, we used a Japanese version of the PIE form and rubric (PIE-J) originally in English. Back translation between English and Japanese was conducted by the main author (YM, Japanese) and an American professor living in Japan literate in both English and Japanese (AJL). PIE stages from all students were assessed by two authors (YM \& MN) by in-depth discussions following the rubric until full agreement was reached.

Responses to all PIE-J questions were investigated for the qualitative analysis. However, we especially focused on PIE-Q9 'Reflect on your experiences in medical school or in the community that have been critical in fostering change in your understanding of what it means to be a professional/to be a physician' to illuminate change processes in PIE stages. 


\section{2) SRL data collection}

Learners' SRL levels were measured by a Japanese-language version of the Motivated Strategies for Learning Questionnaire (MSLQ-J) ${ }^{32}$ in the quantitative analysis reported to be useful in measuring SRL in undergraduate medical education ${ }^{19,33,34}$. MSLQ is composed of 81 items with seven-point Likert scales which quantify levels of nine types of learning strategies (rehearsal: R, elaboration: ELA, organization: O, critical thinking: CT, metacognitive self-regulation: MSR, time and study environment: TaSE, effort regulation: ER, peer learning: PL, and help-seeking: HS), and six variables of motivation states (intrinsic goal orientation: IGO, extrinsic goal orientation: $\mathrm{EGO}$, task value: TV, control of learning beliefs: $\mathrm{CBaL}$, self-efficacy for learning and performance: SEfLaP, and test anxiety: TA). All 81 items were translated into Japanese and backtranslated by the main author (YM) and an American professor (AJL).

\section{Analysis}

\section{1) Quantitative Approach}

Effects of treatment and time (fixed effects) on MSLQ-J scores and PIE-J stages were tested and estimated (RQs 1-2) using two-level regression analysis (upper level: participants; lower level: occasion) in the Open Source statistical package jamovi (version 1.2.9) ${ }^{35}$. Treatment and time were treated as fixed effects (estimated with full informed maximum likelihood), and participantlevel random intercept served as a random effect (estimated with restricted maximum likelihood). For the first measurement of all scales, the two groups were treated as one because the first measurement took place before any treatment (see Chapter 15 in a reference article ${ }^{36}$ for a detailed explanation of this model and the rationale behind it). Marginal $R^{2}$, a multilevel equivalent of the $R^{2}$-statistic commonly used for traditional linear regression models, was used to estimate the effects of time and treatment (values of around $0.01,0.06$ and 0.14 represented small, medium and large effects). The Bayesian Information Criterion (BIC) was used to determine which of the time-effect-only and the time-and-treatment-effect model is to be preferred (i.e., the model with the smallest BIC) ${ }^{36}$. Correlations between MSLQ-J scores and PIF-J stages (RQ3) were analyzed and visualized using network analysis in the Open Source statistical package JASP (version $0.12 \cdot 1 \cdot 0)^{37}$.

\section{2) Qualitative Approach}

From a constructivist paradigm in which 'reality' is subjective and context-specific, and multiple truths are constructed by and between people ${ }^{38}$, qualitative data from PIE-J were analyzed using constructivist thematic analysis. We coded anonymized transcripts of the Japanese essays following the six phases proposed by Braun and Clarke ${ }^{39}$. Coding was conducted by the two Japanese researchers (YM and MN). YM was the lead author, who engaged in the development of PIF-oriented PBL and had experienced qualitative studies relevant to SRL. MN was chosen 
to conduct coding because he is not involved in the JMU curriculum but had experienced qualitative studies relevant to SRL. The PIE statements were thoroughly read and analyzed using an inductive coding approach until agreement on coding was achieved between the pair. In the inductive coding approach, we referred to the PIF stages measured in the quantitative approach. We intensively analyzed statements from those with increased PIE-J stages or intentionally compared statements between those with and without PIF improvement to illuminate meaningful codes and themes. 


\section{Results}

\section{Quantitative data}

Table 5-1 shows the mean \pm SD of 15 MSLQ-J categories in Group A and Group B and the distribution of PIE-J stages.

(a)

\begin{tabular}{|c|c|c|c|c|c|c|c|c|}
\hline ISLQ & Group & Baseline & PBL1 & PBL2 & PBL3 & PBL4 & PBL5 & PBL6 \\
\hline \multirow{2}{*}{ IGO } & A & $3.76 \pm 1.05$ & $3.84 \pm 1.27$ & $3.90 \pm 1.29$ & $4.15 \pm 1.35$ & $4.04 \pm 1.49$ & $4.17 \pm 1.46$ & $4.12 \pm 0.99$ \\
\hline & $B$ & $4.10 \pm 1.25$ & $4.23 \pm 1.00$ & $4.12 \pm 1.33$ & $4.15 \pm 1.17$ & $4.22 \pm 1.27$ & $4.34 \pm 1.64$ & $4.34 \pm 1.25$ \\
\hline \multirow{2}{*}{ EGO } & A & $3.34 \pm 1.29$ & $3.48 \pm 1.33$ & $3.63 \pm 1.43$ & $3.75 \pm 1.43$ & $3.66 \pm 1.56$ & $3.54 \pm 1.53$ & $3.84 \pm 1.38$ \\
\hline & $B$ & $3.68 \pm 1.32$ & $3.69 \pm 1.15$ & $3.82 \pm 1.34$ & $3.94 \pm 1.30$ & $3.91 \pm 1.41$ & $3.94 \pm 1.62$ & $3.81 \pm 1.43$ \\
\hline \multirow{2}{*}{ TV } & A & $4.99 \pm 0.91$ & $4.64 \pm 1.41$ & $4.48 \pm 1.38$ & $4.74 \pm 1.54$ & $4.59 \pm 1.58$ & $4.56 \pm 1.65$ & $4.74 \pm 1.10$ \\
\hline & $B$ & $4.95 \pm 0.97$ & $4.91 \pm 0.93$ & $4.49 \pm 1.29$ & $4.74 \pm 1.33$ & $4.64 \pm 1.44$ & $4.59 \pm 1.77$ & $4.90 \pm 1.31$ \\
\hline \multirow{2}{*}{ CBaL } & A & $4.57 \pm 0.93$ & $4.41 \pm 1.21$ & $4.21 \pm 1.18$ & $4.33 \pm 1.32$ & $4.21 \pm 1.44$ & $4.22 \pm 1.53$ & $4.47 \pm 1.00$ \\
\hline & $B$ & $4.63 \pm 0.99$ & $4.64 \pm 0.83$ & $4.27 \pm 1.27$ & $4.43 \pm 1.21$ & $4.41 \pm 1.30$ & $4.37 \pm 1.59$ & $4.60 \pm 1.20$ \\
\hline \multirow{2}{*}{ SEflaP } & A & $3.00 \pm 1.19$ & $3.42 \pm 1.37$ & $3.51 \pm 1.26$ & $3.64 \pm 1.34$ & $3.66 \pm 1.46$ & $3.92 \pm 1.56$ & $3.75 \pm 1.17$ \\
\hline & $B$ & $3.24 \pm 1.16$ & $3.68 \pm 0.96$ & $3.90 \pm 1.33$ & $4.07 \pm 1.16$ & $4.03 \pm 1.26$ & $4.03 \pm 1.50$ & $3.94 \pm 1.19$ \\
\hline \multirow{2}{*}{ TA } & $A$ & $4.09 \pm 1.24$ & $4.06 \pm 1.28$ & $4.10 \pm 1.43$ & $4.15 \pm 1.41$ & $4.10 \pm 1.60$ & $3.94 \pm 1.59$ & $4.13 \pm 1.30$ \\
\hline & $B$ & $3.79 \pm 1.26$ & $3.90 \pm 1.05$ & $3.83 \pm 1.28$ & $4.03 \pm 1.15$ & $3.75 \pm 1.30$ & $3.83 \pm 1.51$ & $3.99 \pm 1.27$ \\
\hline \multirow{2}{*}{ R } & A & $3.93 \pm 0.90$ & $4.19 \pm 1.20$ & $4.20 \pm 1.22$ & $4.19 \pm 1.32$ & $4.10 \pm 1.43$ & $4.17 \pm 1.45$ & $4.21 \pm 1.03$ \\
\hline & $B$ & $3.79 \pm 1.12$ & $4.13 \pm 0.86$ & $4.15 \pm 1.25$ & $4.05 \pm 1.16$ & $4.12 \pm 1.21$ & $4.42 \pm 1.64$ & $4.37 \pm 1.14$ \\
\hline \multirow{2}{*}{ ELA } & A & $4.72 \pm 0.90$ & $4.47 \pm 1.19$ & $4.39 \pm 1.20$ & $4.55 \pm 1.40$ & $4.48 \pm 1.54$ & $4.41 \pm 1.56$ & $4.57 \pm 1.06$ \\
\hline & $B$ & $4.59 \pm 1.14$ & $4.54 \pm 0.90$ & $4.44 \pm 1.33$ & $4.40 \pm 1.22$ & $4.35 \pm 1.38$ & $4.38 \pm 1.66$ & $4.60 \pm 1.22$ \\
\hline \multirow{2}{*}{0} & $A$ & $4.18 \pm 1.26$ & $4.31 \pm 1.34$ & $4.34 \pm 1.37$ & $4.46 \pm 1.51$ & $4.42 \pm 1.57$ & $41 \pm 1.60$ & $4.40 \pm 1.14$ \\
\hline & $B$ & $4.09 \pm 1.14$ & $4.21 \pm 1.02$ & $4.38 \pm 1.32$ & $4.33 \pm 1.34$ & $4.20 \pm 1.35$ & $4.22 \pm 1.61$ & $4.44 \pm 1.28$ \\
\hline \multirow{2}{*}{ CT } & A & $4.15 \pm 0.85$ & $4.28 \pm 1.14$ & $4.29 \pm 1.27$ & $4.18 \pm 1.32$ & $4.20 \pm 1.51$ & $4.22 \pm 1.47$ & $4.26 \pm 0.88$ \\
\hline & $B$ & $4.14 \pm 1.08$ & $4.20 \pm 0.76$ & $4.22 \pm 1.27$ & $4.09 \pm 1.23$ & $4.12 \pm 1.22$ & $4.14 \pm 1.57$ & $4.36 \pm 1.15$ \\
\hline \multirow{2}{*}{ MSR } & A & $4.06 \pm 0.62$ & $4.22 \pm 0.99$ & $4.26 \pm 1.06$ & $4.24 \pm 1.17$ & $4.25 \pm 1.31$ & $4.27 \pm 1.34$ & $4.28 \pm 0.65$ \\
\hline & $B$ & $4.03 \pm 0.77$ & $4.19 \pm 0.55$ & $4.16 \pm 1.12$ & $4.16 \pm 0.95$ & $4.15 \pm 1.12$ & $4.20 \pm 1.43$ & $4.33 \pm 0.98$ \\
\hline \multirow{2}{*}{ TaSE } & A & $4.25 \pm 0.74$ & $4.23 \pm 0.99$ & $4.21 \pm 1.00$ & $4.46 \pm 1.18$ & $4.28 \pm 1.28$ & $4.29 \pm 1.36$ & $4.37 \pm 0.63$ \\
\hline & $B$ & $4.15 \pm 0.77$ & $4.28 \pm 0.66$ & $4.32 \pm 1.13$ & $4.22 \pm 0.98$ & $4.06 \pm 1.12$ & $4.33 \pm 1.46$ & $4.38 \pm 1.01$ \\
\hline \multirow{2}{*}{ ER } & $A$ & $4.50 \pm 0.89$ & $4.46 \pm 1.17$ & $4.21 \pm 1.07$ & $4.38 \pm 1.20$ & $4.31 \pm 1.35$ & $4.32 \pm 1.41$ & $4.31 \pm 0.63$ \\
\hline & $B$ & $4.28 \pm 0.91$ & $4.49 \pm 0.81$ & $4.26 \pm 1.22$ & $4.21 \pm 1.11$ & $4.14 \pm 1.20$ & $4.31 \pm 1.51$ & $4.29 \pm 1.10$ \\
\hline \multirow{2}{*}{ PL } & A & $4.51 \pm 1.05$ & $4.33 \pm 1.38$ & $4.27 \pm 1.32$ & $4.44 \pm 1.53$ & $4.33 \pm 1.60$ & $4.36 \pm 1.65$ & $4.40 \pm 1.09$ \\
\hline & $B$ & $4.56 \pm 1.27$ & $4.64 \pm 0.94$ & $4.38 \pm 1.42$ & $4.37 \pm 1.32$ & $4.30 \pm 1.43$ & $4.25 \pm 1.73$ & $4.50 \pm 1.32$ \\
\hline \multirow[b]{2}{*}{ HS } & A & $4.46 \pm 0.93$ & $4.36 \pm 1.23$ & $4.23 \pm 1.16$ & $4.32 \pm 1.25$ & $4.25 \pm 1.40$ & $4.34 \pm 1.54$ & $4.29 \pm 0.82$ \\
\hline & $B$ & $4.58 \pm 0.77$ & $4.42 \pm 0.71$ & $4.25 \pm 1.21$ & $4.21 \pm 1.16$ & $4.18 \pm 1.21$ & $4.39 \pm 1.53$ & $4.31 \pm 1.10$ \\
\hline
\end{tabular}

(b)

\begin{tabular}{|c|r|r|r|r|r|r|}
\hline \multirow{2}{*}{$\begin{array}{c}\text { P E-J } \\
\text { stages }\end{array}$} & \multicolumn{3}{|c|}{ G roup A } & \multicolumn{3}{|c|}{ G roup B } \\
\cline { 2 - 7 } & P E 1 & P E 2 & P E 3 & P E 1 & P E 2 & P E 3 \\
\hline II & 7 & 2 & 2 & 6 & 5 & 1 \\
\hline II/ III & 21 & 14 & 15 & 28 & 18 & 15 \\
\hline III & 22 & 20 & 10 & 16 & 26 & 18 \\
\hline III/ IV & 4 & 13 & 19 & 4 & 3 & 13 \\
\hline IV & 0 & 0 & 8 & 0 & 1 & 3 \\
\hline IV/ V & 0 & 0 & 0 & 0 & 0 & 0 \\
\hline $\mathrm{V}$ & 0 & 0 & 0 & 0 & 0 & 0 \\
\hline
\end{tabular}

Table 5-1. (a) The mean \pm SD values of 15 MSLQ-J categories and (b) the distribution of PIE-J stages in Group A and Group B. 
In Table 5-2, a two-level regression analysis showed the $R^{2}$-value of 0.069 in the time-effect-only model in PIE indicated moderate improvement of PIF-J stages over time. However, the timeeffect-only model and the time-and-treatment-effect model yield almost the same marginal $R^{2}$-value and BIC. Therefore, there is no reason to assume treatment effects beyond time-effects only. For the 15 MSLQ scales, we see relatively small differences in score between times (i.e., marginal $R^{2}$-values in the $[0.002,0.034]$ range) and reach the same conclusion about treatment: almost no change in marginal $R^{2}$-value and BIC due to treatment over the time-effect-only model.

Table 5-2. Two-level regression with participant-level random intercept and fixed effects of time and treatment for PIE and the ${ }_{15}$ MSLQ scales: intraclass coefficient (ICC), marginal $R^{2}$-value, and Bayesian information criterion (BIC).

\begin{tabular}{|c|c|c|c|c|c|}
\hline & Random & \multicolumn{4}{|c|}{ Fixed part } \\
\hline \multirow[t]{2}{*}{ Scale } & Intercept & \multicolumn{2}{|c|}{ Time and treatment effect } & \multicolumn{2}{|c|}{ Time effect only } \\
\hline & $\mathrm{ICC}$ & $R^{2}$ & $\mathrm{BIC}$ & $R^{2}$ & BIC \\
\hline PIE & 0.66 & 0.07 & 410.143 & 0.069 & 399.323 \\
\hline \multicolumn{6}{|l|}{ MSLQ } \\
\hline IGO & 0.269 & 0.007 & 10070.636 & 0.005 & 10027.532 \\
\hline EGO & 0.346 & 0.007 & 10420.826 & 0.005 & 10377.412 \\
\hline TV & 0.312 & 0.014 & 14179.596 & 0.012 & 14136.058 \\
\hline $\mathrm{CBaL}$ & 0.221 & 0.011 & 9878.656 & 0.009 & 9832.988 \\
\hline SEfLaP & 0.334 & 0.036 & 19428.128 & 0.034 & 19388.875 \\
\hline TA & 0.253 & 0.002 & 13404.573 & 0.002 & 13357.443 \\
\hline $\mathrm{R}$ & 0.165 & 0.01 & 10571.942 & 0.008 & 10529.513 \\
\hline ELA & 0.292 & 0.006 & 14222 & 0.005 & 14178.355 \\
\hline $\mathrm{O}$ & 0.319 & 0.005 & 9866.504 & 0.004 & 9822.927 \\
\hline CT & 0.263 & 0.003 & 11751.478 & 0.002 & 11703.7 \\
\hline MSR & 0.122 & 0.003 & 29923.339 & 0.003 & 29872.557 \\
\hline TaSE & 0.085 & 0.004 & 22008.123 & 0.002 & 21966.763 \\
\hline ER & 0.157 & 0.005 & 10085.647 & 0.004 & 10041.528 \\
\hline $\mathrm{PL}$ & 0.296 & 0.006 & 7569.559 & 0.004 & 7529.637 \\
\hline $\mathrm{HS}$ & 0.112 & 0.004 & 10977.313 & 0.004 & 10930.299 \\
\hline
\end{tabular}


Figure 5-3 shows the network plot of PIE-J stages and MSLQ-J category scores in the three phases, with thicker lines reflecting stronger correlations than thinner lines, and blue for positive/ red for negative correlations. Overall, correlations between PIE and MSLQ-J scales were rather weak.

Figure 5-3. Network plot of PIE and MSLQ categories.

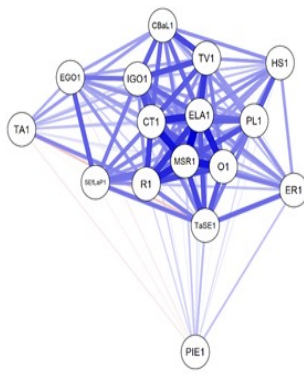

Baseline (PIE1 vs MSLQ1)

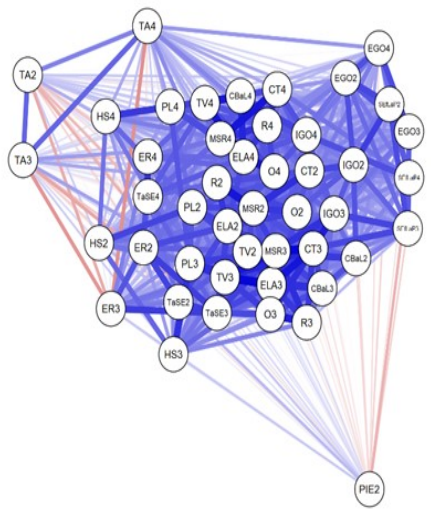

First half (PIE2 vs MSLQ2-4)

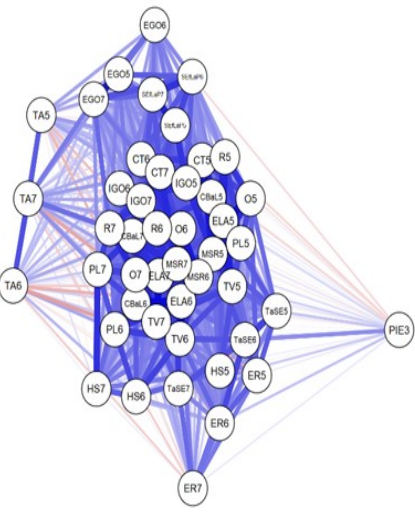

Second half (PIE3 vs MSLQ5-7)

Blue and red lines represent positive and negative correlations, respectively. Thicker lines represent stronger correlations. Weaker lines usually result in longer lines to indicate which variables are more closely related.

\section{Qualitative data}

Table 5-3 shows three themes and representative codes for RQ4 'Why did PIF improve?'. Although the PIF-oriented pre-PBL format did not receive much mention in overall responses, it seemed to contribute to student identity formation as follows. First, the PIF-oriented pre-PBL format helped PBL tasks appear realistic, allowing them to recognize the vast amount of knowledge necessary to make decisions in stressful situations. Second, it helped students articulate their professional images and values, leading to the awareness of unrecognized perspectives regarding medical professionals.

On the other hand, the majority of students described their experiences outside the curriculum, especially during the summer holidays (between PIE 1 and 2 in Figure 5-2). These medical students appeared to use their summer vacation time to visit doctors who are graduates or to take tours of hospitals where they might work in the future. They formed images of 
themselves as physicians through their experiences in the clinical site visits. In other words, professional identity was formed by the real-life experience of being in a clinical setting, recognizing diverse roles and perspectives of the physician, and being impressed by abilities of the clinician beyond medical knowledge and skills.

Table 5-3. Themes and representative codes in thematic analysis for 'why did PIF improve?'

$\begin{array}{ll}\text { Themes } & \text { Subthemes } \\ \text { \# Realistic difficulties that } & \text { \# The vast amount of knowledge for } \\ \text { generate tense emotions } & \text { clinical practice } \\ & \text { \# Encounters with realistic scenes }\end{array}$

Relevant representative codes

\# Realistic experiences in PIForiented PBL

\# Encounters with death and agony from illnesses

\# Awareness of unrecognized

\# Recognition of diverse roles of

\# Encounters with medical perspectives regarding physicians medical professionals

\# Encounters with different professionals in clinical practice in the extracurricular time perspectives that shake-up preNarratives from various medical existing thoughts professionals met in the extracurricular time \# Articulation of professional images and values in PIF-oriented PBL

\# Recognition of expected ability beyond knowledge and skills
\# Recognition of important abilities beyond medical knowledge and skills

\# Patients' expectations of doctors
\# Encounters with physicians in clinical practice in the extracurricular time \# Patients' narratives 


\section{Discussion}

Contrary to the hypothesis, PIF-oriented education for a hybrid PBL in this study did not significantly improve PIF or SRL. Moreover, contrary to the previous study results in clinical contexts ${ }^{18,19}$, PIF levels in this pre-clinical year context did not significantly correlate with SRL. During the seven months in the pre-clinical year, the PIE-J stages of the students improved moderately, but the PIF improvement has contributed little to the improvement in SRL.

According to thematic analysis, students were able to imagine their realistic future scenes where clinicians encounter difficulties through PIF-oriented PBL. They recognized the vast amount of knowledge for medical professionals necessary to make decisions under stressful circumstances. However, outside of the curriculum, what the students encountered in a real medical setting made them recognize not only real difficulties but also the unrecognized aspects of the physician's professionalism and the abilities beyond knowledge and skills needed in clinical practice. As a result, they fostered a multifaceted professional identity beyond the context of PBL.

Many pre-clinical students described extracurricular clinical scenes in PIE. This finding is reasonable. The research period was mostly composed of didactic lectures (approximately 130 hours per month) and written test assessment (18 clinical subjects, three social sciences, and one pathology). Few opportunities to engage in real clinical settings in the formal curriculum might cause students to draw attention to the real settings in their extracurricular experiences.

The thematic analysis of PIE helped us recognize the importance of authentic medical settings to conduct PIF-oriented education. Even one real clinical encounter with a JMUgraduate mentor or a comment from a patient seemed to foster PIF more than actively encouraging students to think about professionalism in a fictional clinical setting of repeated $\mathrm{PBL}$. This is consistent with the results of previous research that shows that future professionalism responsibilities are less likely to be recognized in a non-clinical university setting ${ }^{24-26}$. This study demonstrates the need to give pre-clinical students as much exposure to real clinical situations and communication with real patients as possible. At the same time, the importance of the hidden-curriculum in PIF was also reiterated ${ }^{21,23,24-26}$. Medical educators should consider that the student's daily living environment is part of PIF-oriented education.

Even though we introduced repeated PIF-oriented PBLs in the curriculum, we did only once a month for two hours of pre-learning and eight hours of one-day PBL while approximately 130 hours of didactic lectures per month took place. The PIF-oriented PBL was an attempt to foster SRL within the hybrid PBL in a teacher-centered institutional culture. However, we realize that a relative lack of time for PBL sessions may have led to the lack of expected results. Therefore, fostering pre-clinical student SRL in institutions where teacher-centered culture is prevalent may require extensive curricular reform. 


\section{Strengths}

1. By minimizing threats to internal validity, the experimental setup constitutes an important strength of the study at hand.

2. Subjects were Japanese students placed in a teacher-centered curriculum with teachercentered institutional culture ${ }^{15-19}$. We believe our study can provide educators with evidence that sporadic SRL-oriented educational schemes in such a context cannot improve SRL significantly.

\section{Limitations}

1. This study was conducted in one country and one medical school and for only one year. We would like to expand the content of PIF-oriented PBL further and further verify it at multiple centers.

2. There are no reports that have validated the Japanese version of both MSLQ and PIE. In this study, we used a rigorous back translation by faculty members literate in Japanese and English, but more time examining outcome evaluation methods may be necessary.

3. JMU is a medical school with a mandatory in-dormitory residence for six years, and with a culture in which students frequently share information about their studies in the dormitory. In this study, we permitted only the intervention group students to access the Moodle pre-learning by their IDs and explained in advance that they should not share information with those in the control group. However, some information might have been shared between Groups A and B. A variety of preventive measures can be considered, including investigating at a medical school where students do not board together on campus

\section{Conclusion}

This mixed-method study conducted in a hybrid PBL curriculum showed considerably limited effects of PIF-oriented education on SRL. Within the study period, PIF improved moderately, but not sufficiently to improve SRL. Thematic analysis showed that encounters in extracurricular clinical settings might mainly contribute to PIE improvement. The PIF-oriented PBL was an attempt to foster SRL within hybrid PBL in a teacher-centered institutional culture. However, results suggest a more focused and extensive approach may be necessary. 


\section{List of abbreviations}

JMU: Jichi Medical University; PBL: Problem-based learning; SRL: Self-regulated learning; PIF: Professional identity formation; PIE: Professional identity essay; PIE-J: Japanese version of PIE; MSLQ: Motivated Strategies for Learning Questionnaire; MSLQ-J: Japanese version of MSLQ; IGO: Intrinsic Goal Orientation; EGO: Extrinsic Goal Orientation; TV: Task Value; CBaL: Control Beliefs about Learning; SEfLaP: Self-Efficacy for Learning and Performance; TA: Test Anxiety; R: Rehearsal; ELA: Elaboration; O: Organization; CT: Critical Thinking; MSR: Metacognitive Self-Regulation; TaSE: Time and Study Environment; ER: Effort Regulation; PL: Peer Learning; HS: Help-Seeking.

\section{References}

1. Frank JR. The CanMED 2005 Physician Competency Framework: Better Standards, Better Physicians, Better Care. Ottawa, Canada: Royal College of Physicians and Surgeons of Canada; 2005.

2. Sandars J, Cleary TJ. Self-regulation theory: application to medical education: AMEE Guide No 58. Med Teach. 2011; 33: 875-86.

3. Brydges R, Butler D. A reflective analysis of medical education research on self-regulation in learning and practice. Med Educ. 2012; 46: 71-9.

4. Berkhout JJ, Helmich E, Teunissen PW, Van der Vleuten CP, Jaarsma ADC. Context matters when striving to promote active and lifelong learning in medical education. Med Educ. 2018; 52: 34-44.

5. Zimmerman BJ. A social cognitive view of self-regulated academic learning. Journal of Educ Psychol. 1989; 81:329-39.

6. Artino AR, Jones KD. AM Last Page: Self-regulated learning—a dynamic, cyclical perspective. Acad Med. 2013; 88: 1048.

7. Zimmerman BJ: Attaining self-regulation: A social-cognitive perspective. In Handbook of SelfRegulation. Edited by: Boekaerts M, Pintrich P, Ziedner M. San Diego, CA, USA: Academic Press; 2000: 13-39.

8. van Houten-Schat MA, Berkhout JJ, van Dijk N, Endedijk MD, Jaarsma ADC, Diemers AD. Selfregulated learning in the clinical context: a systematic review. Med Educ. 2018; 52: 1008-15.

9. Berkhout JJ, Teunissen PW, Helmich E, van Exel J, van der Vleuten CP, Jaarsma DA. Patterns in clinical students' self-regulated learning behavior: a Q-methodology study. Adv Health Sci Educ Theory Pract. 2017; 22: 105-21.

10. Woods NN, Mylopoulos M, Brydges R. Informal self-regulated learning on a surgical rotation: uncovering student experiences in context. Adv Health Sci Educ Theory Pract. 2011; 16: 643-53.

11. Barrows HS, Tamblyn R. Problem-based learning: an approach to medical education. New York: Springer; 1980.

12. Loyens SM, Magda J, Rikers RM. Self-directed learning in problem-based learning and its relationships with self-regulated learning. Educ Psychol Rev. 2008; 20: 411-27

13. Sungur $S$, Tekkaya C. Effects of problem-based learning and traditional instruction on selfregulated learning. J Educ Res. 2006; 99: 307-20. 
14. Kwan CY. A thorny path: the developmental course of problem-based learning for health sciences education in Asia. Adv Health Sci Educ Theory Pract. 2019; 24: 893-901.

15. Yoshioka T, Suganuma T, Tang AC, Matsushita S, Manno S, Kozu T. Facilitation of problem finding among first year medical school students undergoing problem-based learning. Teach Learn Med. 2005; 17: 136-41.

16. Shimizu I, Nakazawa H, Sato Y, Wolfhagen IHAP, Könings KD. Does blended problem-based learning make Asian medical students active learners?: a prospective comparative study. BMC Med Educ. 2019; 19: 147.

17. Iwata K, Doi A. Can hybrid educational activities of team and problem based learning program be effective for Japanese medical students?. Kobe J Med Sci. 2017; 63: E51-7.

18. Matsuyama Y, Nakaya M, Okazaki H, Lebowitz AJ, Leppink J, van der Vleuten CPM. Does changing from a teacher-centered to a learner-centered context promote self-regulated learning: a qualitative study in Japanese undergraduate setting. BMC Med Educ. 2019; 19: 152.

19. Matsuyama Y, Okazaki H, Kotani K, Asada Y, Ishikawa S, Lebowitz AJ, Leppink J, van der Vleuten C. Education in professional identity formation enhances self-regulated learning: a mixedmethod explanatory study from a community-based clinical clerkship in Japan. Under review.

20. Cruess RL, Cruess SR, Boudreau JD, Snell L, Steinert Y. Reframing medical education to support the development of a professional identity. Acad Med. 2014; 89: 1446-51.

21. Wald HS, Anthony D, Hutchinson TA, Liben S, Smilovitch M, Donato AA. Professional identity formation in medical education for humanistic, resilient physicians: pedagogic strategies for bridging theory to practice. Acad Med. 2015; 90: 753-60.

22. Barnhoorn PC, Houtlosser M, Ottenhoff-de Jonge MW, Essers GTJM, Numans ME, Kramer AWM. A practical framework for remediating unprofessional behavior and for developing professionalism competencies and a professional identity. Med Teach. 2019; 41: 303-8.

23. de Lasson L, Just E, Stegeager N, Malling B. Professional identity formation in the transition from medical school to working life: a qualitative study of group-coaching courses for junior doctors. BMC Med Educ. 2016; 16: 165.

24. Stockley AJ, Forbes K. Medical professionalism in the formal curriculum: 5th year medical students' experience. BMC Med Educ. 2014; 14: 259.

25. Goldie J. The formation of professional identity in medical students: considerations for educators. Med Teach. 2012; 34: e641-8.

26. Baernstein A, Oelschlager AM, Chang TA, Wenrich MD. Learning professionalism: perspectives of pre-clinical medical students. Acad Med. 2009; 84: 574-81.

27. Kozu T. Medical education in Japan. Acad Med 2006; 81: 1069-75.

28. Kalet A, Buckvar-Keltz L, Harnik V, Monson V, Hubbard S, Crowe R, Hyuksoon SS, Yingling S. Measuring professional identity formation early in medical school. Med Teach. 2017; 39: 255-61.

29. Kegan R. In over our heads: The mental demands of modern life. Cambridge, MA, USA: Harvard University Press; 1994.

30. Creswell J. Mixed methods designs. In Educational Research. 4th ed. Edited by: Creswell J. Boston, USA: Pearson; 2012: 564-605.

31. Kalet A, Buckvar-Keltz L, Monson V, Harnik V, Hubbard S, Crowe R, Ark T, Song H, Tewksbury L, Yingling S. Professional Identity Formation in medical school: one measure reflects changes during pre-clerkship training. MedEdPublish. 2018; 7.

32. Pintrich PR, Smith D, Garcia T, McKeachie WJ. A manual for the use of the Motivated Strategies for Learning Questionnaire (Technical Report 91-B-004). Michigan, USA: The Regents of the University of Michigan; 1991. 
33. Cho KK, Marjadi B, Langendyk V, Hu W. Medical student changes in self-regulated learning during the transition to the clinical environment. BMC Med Educ. 2017; 17: 59.

34. Kim KJ, Jang HW. Changes in medical students' motivation and self-regulated learning: a preliminary study. Int J Med Educ. 2015; 6: 213-5.

35. Jamovi project. Jamovi version 1.2.9. Retrieved Apr 21, 2020, from https://www.jamovi.org/

36. Leppink J. Statistical methods for experimental research in education and psychology. Springer, Cham; 2019. https://doi.org/10.1007/978-3-030-21241-4

37. Love J, Selker R, Marsman M, et al. JASP version 0.12.1.0. Retrieved Apr 21, 2020, from https:// jasp-stats.org/

38. Bergman E, de Feijter J, Frambach J, Godefrooij M, Slootweg I, Stalmeijer R, van der Zwet J. AM last page: A guide to research paradigms relevant to medical education. Acad Med. 2012; 87: 545.

39. Braun V, Clarke V. Using thematic analysis in psychology. Qual Res Psychol. 2006; 3: 77-101. 

Chapter 6

Discussion 
This thesis has sought to explore the challenges of self-regulated learning (SRL) in teachercentered contexts. In particular, overarching research questions pertaining to the challenges of SRL in teacher-centered contexts were asked:

\section{What contextual attributes could keep learners in a teacher-centered context from becoming self-regulated learners?}

2. What contextual attributes could be triggers for learners in a teacher-centered culture to become self-regulated learners?

3. Can we stimulate SRL when introducing education with SRL-triggering attributes?

This chapter will start by considering the answers that were found to these questions, linking the findings to other work and discussing the implications for medical education. The strengths and limitations of the thesis will be considered and suggestions will be proposed for further research in this area.

\section{What contextual attributes could keep learners in a teacher-centered context from becoming self-regulated learners?}

In the midst of various changes in medicine, such as advances in medical science and technology, changes in social systems, diversification and increasing social needs for medical care, and globalization, medical educators around the world are trying to break away from the traditional curriculum and build a new one $\mathrm{e}^{1-4}$.

As one of the strategic tools for planning new curricula or evaluating existing ones, the SPICES model draws a contrast in contextual attributes between the conventional curriculum and the new curriculum5. According to the SPICES model, contextual attributes in the traditional curriculum include teacher-centered, information-gathering, discipline-based, hospital-based, standard program, and apprenticeship-based or opportunistic, while those in the new curriculum include student-centered, problem-based, integrated, community-based, electives, and systematic.

However, few studies in the context of medical education have delineated how students are actually motivated to learn and what learning strategies they use within such a traditional curriculum. In the study presented in Chapter 2 of this thesis, we were able to identify which contextual attributes of teacher-centered learning influence students' learning behaviors and learning emotions by asking students studying under a teacher-centered curriculum in an extant East Asian medical school. In particular, the strength of this study is that by applying the SRL theory advocated by Zimmerman ${ }^{6}$, the contextual attributes could be more clearly described. This is because the components of the distinct learning features were used as criteria, namely 'motivation', 'learning strategy' and 'reflection on learning' were used as criteria. The contextual 
attributes of teacher-centered learning were further characterized by contrasting them with the learning contexts of populations that completed the teacher-centered curriculum and subsequently became independent rural physicians as self-regulated learners, even within the same culture. In the study presented in Chapter 3, in the seven-month exposure of learning contextual attributes of students who continued a teacher-centered curriculum versus those who moved to a student-centered curriculum in the same period of time and in the same grade at the same university, we were able to clarify the learning characteristics of each learner through a process of contrast, based on the aforementioned three components of SRL.

A strength of this project's results is its replication of the contextual attributes of the teachercentered curriculum in Chapters 2 and Chapter 3. In other words, individual students in the teacher-centered curriculum seemed to motivate themselves to remain part of the participating groups in the curriculum (e.g., classmates, practicum groups), to reflect on themselves in contrast to the standards set by the teacher, and to engage in monotonous learning behaviors tailored to their "less failing" surroundings that did not deviate from the standards of the group or the teacher. An example of monotonous learning is remembering the teacher's preferred study tasks as they were taught in order to minimize the possibility of failing tests and not to stray from the group. This learning strategy is the closest to "rehearsal" in SRL, and is often derided by the term "memorization" because it is unlike knowledge-building learning strategies such as elaboration and organization ${ }^{78,9}$. 'Memorization' is often categorized into surface learning as the opposite of deep learning or meaningful learning ${ }^{9,10}$.

The 'memorization' of learning among East Asian students has been discussed. Kember et al. ${ }^{11}$ use the term 'narrow orientation' to describe the causes of dissociation in memorizationbased learning and high performance on academic tests among East Asian high school students. In other words, these students are trying to narrow down the learning target of the given material and memorize what is being written while trying to understand it. Purdie et al..$^{12}$ also describe how Japanese high school students use repetitive memorization to reach a meaning-oriented understanding. Indeed, based on these studies, their memorization may have implications for learning strategies beyond surface learning. However, these studies are only in the context of learning up to high school. In the context of medical school, where vocational training is an aspect of education that prepares individuals to learn while actively participating in social activities as professionals, this type of learning strategy has its drawbacks.

For example, Iwata et al. ${ }^{13}$ reported that fourth-year students in a Japanese medical school working in small groups using clinical cases expressed dissatisfaction with learning something they had not learned in class. Rather, they strongly desired to utilize memorization through teacher lectures, and imitated or read out the answers of 'super-achievers' in small groups. Matsuyama et al. ${ }^{14}$ reported that Year 2 to 5 medical students in Japan lost motivation when they were asked about a medically important subject that they had not yet learned in class, and wanted a teacher's lecture when reviewing the exam. Watanabe et al. ${ }^{15}$ also reported that Japanese medical students study in a narrow framework based on the value of studying for examinations. 
Notably, all three of these papers make the point that students strongly rely on an education with hands-on teacher guidance to win the tough examination war before entering university.

Learning in the medical field is about preparing for problems that cannot, or can only to a limited extent, be addressed by previous existing knowledge. Medical knowledge is vast and the ability to simply memorize it no longer makes much contribution. Rather, one must regulate a motivation, and select a way to approach necessary and accurate information in an active way ${ }^{16-19}$. Physicians may encounter an urgent situation in which they cannot wait for supervising physicians to teach them. The qualification of being a doctor is something that is given to the individual. When the renewal of professional competence as a physician is viewed in terms of the physician's lifetime, it is nothing more than an active act of the individual over time ${ }^{16,19}$. Therefore, no matter how much memorization in East Asia might have greater function than in the West in the preuniversity context, it is a weak foundation for lifelong learning for medical professionals.

The studies described in Chapters 2 and 3 replicate the results of these previous studies ${ }^{13-15}$ within the theoretical framework of SRL. In other words, if students who have only experienced learning by being taught and taught enter medical schools and are given a large amount of teaching materials, if the curriculum is composed mainly of lectures by teachers, and if examination results are the main requirement for advancement, a major change in learning strategies is unlikely to occur.

On returning to the research questions in this section, we could conclude that medical students who were familiar with the teacher-centered curriculum before entering school i) maintain motivation to remain a member of a group governed by the teacher's standards of values, ii) reflect the standards set by the teacher, and iii) study the learning materials set by the teacher in a uniform manner. These are the contextual attributes that could keep learners in a teacher-centered context from becoming self-regulated learners.

\section{What contextual attributes could be triggers for learners in a teacher-centered culture to become self-regulated learners?}

A key outcome of Chapter 2 was the contrast between motivational, reflective, and learning strategies in self-learning between learners in teacher-centered undergraduate curricula and those in educationally under-resourced settings in postgraduate rural medical practice. Overall, contextual attributes can promote SRL even among East Asian populations, who are believed to lack SRL during undergraduate education. Therefore, we emphasize that insufficient SRL should not be attributed to the cultural values of education, but rather to curricula that are filled with teacher-centered evaluations and do not encourage learners to proactively envision their own individual future as doctors.

By contrasting the two contexts in the same culture in the Chapter 2 study, we were able to show that fundamental contextual attributes which influence SRL are independent of cultural 
characteristics. Therefore, the research in Chapter 3 was carried out in a similar manner. That is, the study contrasted SRL between students who remained in the teacher-centered curriculum and those who moved to the learner-centered curriculum at the same university, during the same school years, and over the same period of time in the same teacher-centered education culture. The results of Chapter 3 show the imperatives required to promote SRL in the teacher-centered curriculum. Namely, students must: 1) get away from the classroom lecture and exam-oriented environment imposed by the faculty, and envision their own future as a professional, 2) reflect on their future image in comparison with their present situation, and 3) emulate various learning strategies shown by role models to bridge the gap.

On integrating the results of the two research settings in Chapter 2 and Chapter 3, the following learning contextual attributes appear to be triggers for learners in a teacher-centered culture to become self-regulated learners: i) have individuals develop their own unique future professional identity, ii) have them compare their future and current selves and relate the gapfilling objectives to the learning tasks in front of them, and iii) experience a variety of learning strategies presented from role models that are aligned with the learning tasks in front of them.

We applied the term "professional-identity formation (PIF) ${ }^{20}$ " to describe the first contextual attribute because of its popularity and applicability in medical education. PIF is defined as the formation of "a representation of self, achieved in stages over time during which the characteristics, values, and norms of the medical profession are internalized ${ }^{20}$." From the results of Chapters 2 and 3, it can be said that PIF is central to the SRL-triggering attributes in this question. Therefore, we propose a theory that PIF can be a trigger for SRL development in teacher-centered educational cultures, and name it 'PIF-based SRL theory' (Figure 6-1).

Figure 1 The concept map of PIF-based SRL theory

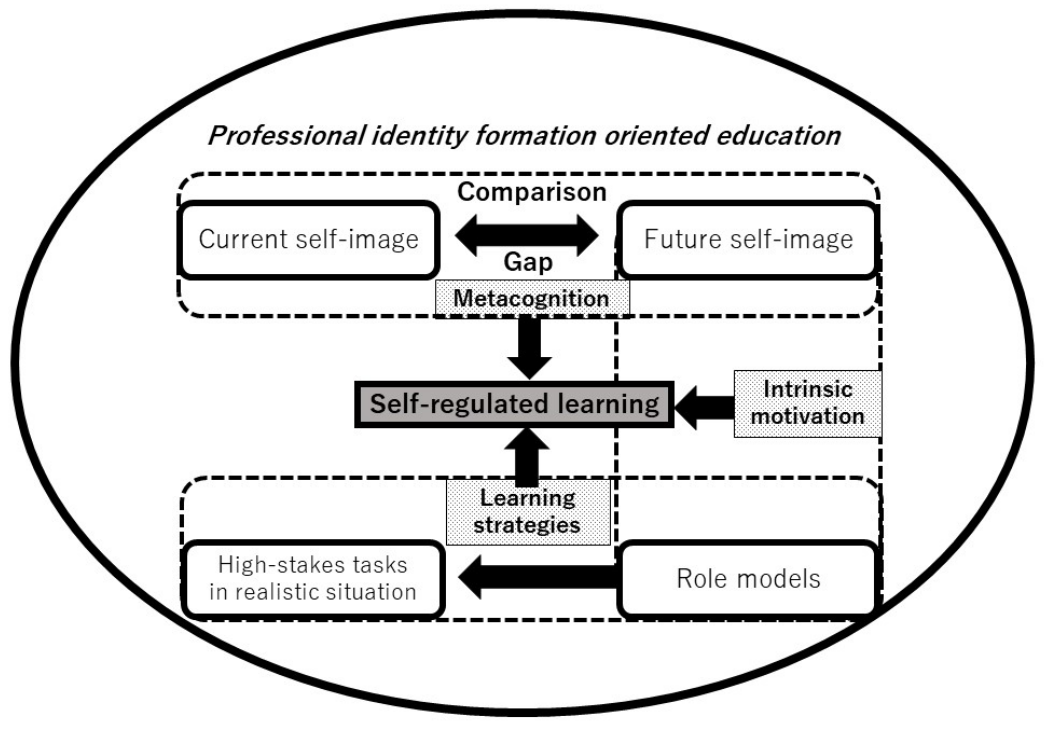


The professional identity formation (PIF)-oriented education proposed in this dissertation encompasses the contrast between the present and future self, interaction with role models that overlap with the future self, and the lessons of learning strategies from role models who learn highstakes challenges in realistic situations. That is, PIF-oriented education enhances SRL, including contextual attributes related to metacognition, intrinsic motivation, and learning strategies.

The central topic in the following part of this chapter is what kind of educational strategies are needed to apply this theory to teacher-centered settings. The discussion on this topic will be applied to answering the third research question in the next section.

\section{Can we change learners' self-regulation in learning when introducing education with SRL-triggering attributes?}

In order to translate the theory into practice, a format for intervention education based on this PIF-based SRL theory was developed and its usefulness was tested in two learning environments in Chapters 4 and Chapter 5. Since most medical school curricula separate the clinical practice period and the pre-clinical practice period, we set up an examination in each, with Chapter 4 being the examination in the clinical practice period and Chapter 5 being the examination in the pre-clinical practice period.

First, we discuss Chapter 4. At the research site or Jichi Medical University (JMU), all students are required to work at a hospital in a remote area with limited medical and educational resources for about seven years after graduation ${ }^{21,22}$. This should foster an identity of working as a general practitioner in community medicine in all JMU medical students. We considered that the PIFbased SRL theory could be appropriately validated in the rural community-based clinical clerkship at JMU or a learning environment where undergraduates will work in future with alumni directly mentoring them. Notwithstanding the short duration of the intervention, the mixed-method study with a comparative approach brought about improvements in intrinsic goal orientation and critical thinking, both quantitatively and qualitatively. Moreover, in the quantitative part, imagining oneself doing the work and relating study subjects to the working process proved to be an established SRL skill that included an element of elaboration ${ }^{7,8}$.

One of the weaknesses of the study in Chapter 4 was that it did not measure the actual improvement in the subject's PIF, which is the basis of the theory. With this in mind, the study in Chapter 5 was designed using both PIF and SRL measurement tools. For the setting of the Chapter 5 study, we chose a hybrid PBL that is used around East Asia, albeit at the expense of the limiting SRL improvement ${ }^{23}$. We conducted an explanatory mixed-method to explore the background of the results obtained in the randomized controlled trial. Eventually, we found that educational interventions based on the PIF-based SRL theory were not sufficient to improve PIF or SRL in the pre-clinical year and in a hybrid PBL setting. However, what was further explored 
in the qualitative arm was that students' voluntary actions outside of the curriculum contributed significantly to the improvement in PIF, as a background factor for the improvement in students' PIF either with or without the intervention. Surprisingly, students who were considered to be passive learners in the teacher-centered curriculum were actively seeking out and participating in experiential learning during long holidays outside of the curriculum. They went to meet graduates at medical institutions where they would work in the future.

The differences in the results of the intervention studies in Chapters 4 and 5 prompt the following considerations. In the case of Chapter 5, an SRL-oriented intervention was sporadically provided within seven months of the hybrid PBL, but the environment in which PIF was actually fostered was outside the curriculum. Such a dissonance between intervention tasks and learning environment might lead to unexpected outcomes. The SRL-oriented intervention held around the rural community-based clinical clerkship in Chapter 4 was conducted in a single session, but there was resonance between the PIF-oriented learning tasks and the environment in which PIF could improve.

All in all, returning to the research questions in this section, we could say, 'Yes, we can stimulate learners' SRL when introducing education with SRL-triggering attributes or PIFbased SRL theory. However, as a condition for this answer, we would have to add that 'learning tasks based on PIF-based SRL theory must not be given in classroom settings, in which PIF hardly improves'.

\section{Implications for medical education}

When consolidating all of the notions in the previous sections, we emphasize that the development of SRL through PIF can be achieved by drastically reducing the number of lectures and increasing the number of classes in clinical settings, even in areas that are believed to have teacher-centered cultures. In addition, unless PIF-oriented practice is carried out at the clinical site, it will be very difficult to escape from teacher-dependent learning in the current preclinical year curriculum. This may be because students were fully immersed in teacher-instructed reproductive learning until they entered university, and it will be extremely difficult to foster SRL without them developing self-image of medical professionals-to-be.

Looking at efforts we have made so far to foster SRL in a teacher-centered culture made, it appears as if we have been expecting to foster SRL by transplanting only the framework of teaching methods from the West. This was mistaken, however, because PBL was only superficially transplanted and did not fully penetrate into the curriculum ${ }^{23}$. What is needed now is a fundamental shift in stance to foster the professional identity of the learner, rather than forcing learners to experience only new teaching methods. We propose that the PIF-based education confirmed in this study is an essential strategy in developing SRL that will lead to lifelong active learning skills, even in a teacher-centered culture. 
The strategy for future curriculum reform will have to be cautious yet bold. By cautious, we mean that the curriculum of Western cultures should be used as a reference, but that superficial and partial copies of the curriculum without cautious consideration should be avoided in order not to repeat the failure of the introduction of PBL. For example, an integrative, longitudinal, workplace-based, early clinical experience programs in the West can be a model for a curriculum that is equipped with a conducive environment for the PIF in this study ${ }^{24,25}$. This means that, starting in the lower grades, the students go to community hospitals on a regular basis, and over the course of a long period of time, they develop a real sense of the role of a health care provider while facing real patients and role models. We would emphasize that this should not be part of the curriculum, but should be introduced into the curriculum over time, similarly to or - ideally - more so than in the West to boldly move away from an environment of teacher-centered learning.

As the study in Chapter 3 shows, we will also be able to implement a course in which students plan their learning in a student-centered way for several months away from teacher-centered lecture classes. The Free Course Student Doctor at JMU might be a good platform to provide opportunities which foster PIF-based SRL. Another option can be found in Chapter 5. Preclinical students in Japan were active over the summer holidays, going to meet their role models and visit the medical sites where they would be working in the future. Having students visit the medical sites where they will work in the future regularly within the preclinical curriculum therefore appears to be effective in fostering PIF and SRL. In particular, creation of a class in which students call on graduates working as doctors at a medical practice would appear effective.

\section{References}

1. Quirk ME, Harden RM. Curriculum planning and development. In A Practical Guide for Medical Teachers. Edited by: Dent J, Harden R, Hunt D. London, UK: Elsevier; 2017: 4-12.

2. O'Brien BC, Forrest K, Wijnen-Meijer M, ten Cate O. A Global View of Structures and Trends in Medical Education. In Understanding Medical Education: Evidence, Theory, and Practice, Third edition. Edited by: Swanwick T, Forrest K, O’Brien BC. 2019: 7-22.

3. Powell DE, Carraccio C. Toward competency-based medical education. N Engl J Med. 2018; 378: 3-5.

4. Schwartzstein RM, Roberts DH. Saying goodbye to lectures in medical school—paradigm shift or passing fad. N Engl J Med. 2017; 377: 605—7.

5. Harden RM, Sowden S, and Dunn WR. Educational strategies in curriculum development: the SPICES model. Med Educ. 1984; 18: 284-97.

6. Zimmerman BJ. A social cognitive view of self-regulated academic learning. J Educ Psychol. 1989; 81:329-39.

7. Pintrich PR, Smith DA, Garcia T, McKeachie WJ. Reliability and predictive validity of the Motivated Strategies for Learning Questionnaire (MSLQ). Educ Psychol Meas. 1993; 53: $801-13$. 
8. Vermunt JD. Relations between student learning patterns and personal and contextual factors and academic performance. High Educ. 2005; 49: 205-34.

9. Dolmans DH, Loyens SM, Marcq H, Gijbels D. Deep and surface learning in problem-based learning: a review of the literature. Adv Health Sci Educ Theory Pract. 2016; 21:1087-112.

10. Biggs JB, Kember D, Leung DYP. The revised two-factor study process questionnaire: R-SPQ-2F. Brit J Educ Psychol. 2001; 71: 133-49.

11. Kember D. The intention to both memorise and understand: Another approach to learning?. High Educ. 1996; 31: 341-54.

12. Purdie N, Hattie J, Douglas G. Student conceptions of learning and their use of self-regulated learning strategies: A cross-cultural comparison. J Educ Psychol. 1996; 88: 87-100.

13. Iwata K, Doi A. Can Hybrid Educational Activities of Team and Problem Based Learning Program be Effective for Japanese Medical Students? Kobe J Med Sci. 2017; 63: E51-7.

14. Matsuyama Y, Muijtjens AM, Kikukawa M, Stalmeijer R, Murakami R, Ishikawa S, Okazaki H. A first report of East Asian students' perception of progress testing: a focus group study. BMC Med Educ. 2016; 16: 245.

15. Watanabe Y, Fujimoto S, Shibahara M, Otaki J. Self-directed learning for first year medical students; for bridging the gap between entrance examinees and beginners at clinical learning. J Nara Med Assoc. 2018; 69: 27-42. (Japanese)

16. Frank JR. The CanMED 2005 Physician Competency Framework: Better Standards, Better Physicians, Better Care. Ottawa, Canada. Royal College of Physicians and Surgeons of Canada; 2005.

17. Sandars J, Cleary TJ. Self-regulation theory: application to medical education: AMEE Guide No 58. Med Teach. 2011; 33: 875-86.

18. Brydges R, Butler D. A reflective analysis of medical education research on self-regulation in learning and practice. Med Educ. 2012; 46: 71-9.

19. World Federation for Medical Education. Continuing Professional Development (CPD) of Medical Doctors: WFME Global Standards for Quality Improvement. University of Copenhagen. 2003.

20. Cruess RL, Cruess SR, Boudreau JD, Snell L, Steinert Y. Reframing medical education to support the development of a professional identity. Acad Med. 2014; 89: 1446-51.

21. Matsumoto M, Kajii E. Medical education program with obligatory rural service: analysis of factors associated with obligation compliance. Health Policy. 2009; 90: 125-132.

22. Matsumoto M, Inoue K, Kajii E. Long-term effect of the home prefecture recruiting scheme of Jichi Medical University, Japan. Rural Remote Health. 2008; 8: 930.

23. Kwan CY. A thorny path: the developmental course of problem-based learning for health sciences education in Asia. Adv Health Sci Educ Theory Pract. 2019; 24: 893-901.

24. Dornan T, Littlewood S, Margolis SA, Scherpbier A, Spencer J, Ypinazar V. How can experience in clinical and community settings contribute to early medical education? A BEME systematic review. Med Teach. 2006; 28: 3-18.

25. McGeehan J, English R, Shenberger K, Tracy G, Smego R Jr. A community continuity programme: volunteer faculty mentors and continuity learning. Clin Teach. 2013;10: 15-20. 

Summary 


\section{Chapter 1}

Clinical knowledge is rapidly advancing. Medical professionals need to update their knowledge autonomously throughout their professional life. Therefore, self-regulation in learning has come to be an important competency for medical professionals throughout life. To date, scholars have been vigorously examining self-regulation in learning under the term "self-regulated learning (SRL)", which is defined by three components, as "learners' active participation in their own learning process from metacognitive, motivational, and behavioural perspectives."

A number of studies have emphasized that the learning context determines whether learners engage in SRL. However, most of these studies were conducted in Western cultural contexts. When educational researchers emphasize the importance of contextual attributes in SRL worldwide, increased focus should be placed on cultural context. Education in East Asia is often referred to as Confucian-heritage education, characterized by family members, teachers, and society by an overemphasis on passing examinations, obtaining higher grade points and ranking in a higher position to assure future success. Medical education in East Asia is also influenced by Confucian-heritage education. Students in East Asia reportedly prefer "learning by being taught and given." Teacher-centered learning occupies the curriculum, which is dominated by teacher guidance and evaluation by teachers; in contrast, student-centered learning is encouraged internationally.

The delayed development of SRL in medical students is problematic because residency training cannot prepare residents for every challenge their qualification brings. Therefore, the goal of this research project was to identify underlying problems: namely, what delays the development of SRL, and how medical education can improve the delays. We conducted four separate studies in line with the following three research questions:

\section{What contextual attributes could keep learners in a teacher-centered context from becoming self- regulated learners?}

2. What contextual attributes could be triggers for learners in a teacher-centered context to become self-regulated learners?

\section{Can we stimulate SRL when introducing education with SRL-triggering attributes?}

\section{Chapter 2}

The study described in Chapter 2 sought to address the first and second research questions by exploring the contrast in self-study between two explicitly different learning contexts in a teacher-centered culture, namely Japan. We identified a group of self-regulated learners in a 
unique postgraduate context (a rural medical context), who used SRL even though they had received teacher-centered undergraduate education in Japan. By contrasting their undergraduate context with their rural context in daily self-study, we sought to explore contextual attributes promoting or hampering SRL.

We conducted interviews and diary data collection among rural physicians $(n=10)$ and undergraduates $(n=11)$ in Japan who undertook self-study of unfamiliar diseases. We analyzed three domains of Zimmerman's definition of SRL: learning behaviors, motivation, and metacognition using constructivist grounded theory.

As a result, we found that rural physicians recognized their identity as unique, and as professionals with a central role in handling diseases in the local community by conducting selfstudy. They simultaneously found themselves at risk of providing inappropriate aid if their selfstudy was insufficient. They developed various learning strategies to cope with this high-stakes task. Undergraduates had a fear of being left behind and preferred to remain as one of the crowd with students in the same school year. Accordingly, they copied the methods of other students for self-study and used monotonous and homogeneous strategies.

In conclusion, we established that different learning contexts do not keep East Asian learners from being self-regulated. Awareness of their unique identity leads them to view learning tasks as high-stakes, and to initiate learning strategies in a self-regulated manner. On the other hand, teacher-centered education systems cause students to identify themselves as one of the crowd, and tasks as low-stakes, and to accordingly employ non-self-regulated strategies.

\section{Chapter 3}

The study in Chapter 2 indicated that a teacher-centered context could hinder undergraduates from SRL, whereas a learner-centered context could promote it. However, SRL development between a teacher-centered and a learner-centered context has not been directly compared in undergraduate settings. Also, it is still unclear how a contextual change toward learner-centered learning could influence SRL in students who are strongly accustomed to teacher-centered learning.

We conducted three focus groups that examined 13 Japanese medical students who left a traditional curriculum composed of didactic lectures and frequent summative tests and entered a seven-month elective course (Free Course Student Doctor or FCSD). The FCSD emphasizes student-designed individualized learning with support and formative feedback from mentors chosen at the students' discretion. We also conducted two focus groups that examined 7 students who remained in the teacher-centered curriculum during the same period. Students were asked to discuss their 1) motivation, 2) learning strategies, and 3) self-reflection on self-study before and during the period. Data were analyzed using thematic analysis and code comparison between the two cohorts. 
The results showed that non-FCSD participants described their motivational status as being one among a crowd set by the teacher's yardstick. Their reflection focused on minimizing the gap between themselves and the teacher-set yardstick with strategies considered monotonous and homogeneous (e.g. memorization). FCSD participants described losing the teacher-set yardstick and constructing their future self-image as an alternative yardstick. They compared gaps between their present status and future self-image by self-reflection. To fill these gaps, they actively employed learning strategies used by doctors or mentors, leading to diversification of their learning strategies.

In conclusion, a contextual change toward learner-centered learning could promote SRL even in students strongly accustomed to teacher-centered learning. In the learner-centered context, students began to formulate professional identity, conduct self-reflection, and seek diverse learning strategies by referring to future 'self' models.

\section{Chapter 4}

Studies in Chapter 2 and 3 found that a change in context to one where students can formulate a professional identity might be important for SRL improvement. We applied the term "professional-identity formation (PIF)" to describe the contextual attribute, based on its popularity and applicability in medical education. However, the studies in Chapter 2 and 3 failed to demonstrate a cause-effect relationship between PIF-oriented context and SRL improvement. Therefore, we created a PIF-oriented education format based on study findings illustrated in Chapter 2 and 3 , and investigated the effects of this PIF-oriented education on SRL in students in their clinical years.

Chapter 4 describes a mixed-method study which explored the effects of a PIF-oriented education format designed to help undergraduates 1) articulate a future 'self' image as a professional and 2) have in-depth communication pertaining to medical professionalism with role models in a clinical clerkship. The clinical clerkship setting was a rural community-based clinical clerkship (rCBCC) for students of Jichi Medical University (JMU), in which clinical year students study in their future workplace under the direct education of graduates (role models). Forty-one Y5 JMU students experienced PIF-oriented rCBCC, while 41 Y5 control subjects experienced the original format $\mathrm{rCBCC}$. Changes in SRL between the two groups were compared using 15 categories of the Motivated Strategies for Learning Questionnaire (MSLQ). We also explored how the PIF-oriented rCBCC changed their SRL by thematic analysis of questionnaire and interview data.

As a result, a moderate improvement in intrinsic goal orientation $\left(p=0.005, \varepsilon^{2}=0.096\right)$ and a mild improvement in critical thinking $\left(p=0.041, \varepsilon^{2}=0.051\right)$ were observed in the PIF-oriented rCBCC. Qualitative analysis revealed that they started viewing 'professional responsibility' as a self-study motivator. Awareness of 'responsibility' led to the pursuit of authenticity and medical 
knowledge applicability, and fostered critical thinking about learning contents. They also began elaborating learning contents in line with professional task processes.

The study in Chapter 4 showed some effects of PIF-oriented education on SRL improvement. Fostering PIF may be important for stimulating learners' intrinsic SRL behaviors.

\section{Chapter 5}

Chapter 5 describes a mixed-method study which explored the effects of a PIF-oriented education format designed for a preclinical hybrid PBL curriculum in a teacher-centered culture. We created the PIF-oriented pre-PBL learning format to help preclinical year students 1) articulate their future 'self' image as a professional and 2) have in-depth communication with role models pertaining to medical professionalism before every PBL session.

In the quantitative part, a randomized controlled crossover trial was conducted using 112 preclinical year students at JMU. In six one-day PBL sessions, Groups A ( $n=56$, female 18 , male 38 , mean age \pm SD $21.5 \pm 0.7)$ and $\mathrm{B}(n=56$, female 11 , male 45, mean age \pm SD $21.7 \pm 1.0)$ experienced PIF-oriented pre-PBL learning, three times each in the first and second halves of the year. PIF and SRL levels between the two groups were compared using the Professional Identity Essay (PIE) and Motivated Strategies for Learning Questionnaire (MSLQ), respectively. In the qualitative part, thematic analysis of what brought about an improvement in PIF in the research period was performed using the PIE description.

Two-level regression analysis showed moderate improvement in PIF stages over time $\left(R^{2}=\right.$ $0.069)$, regardless of intervention. PIF-oriented pre-PBL learning alone did not significantly improve PIE stage or MSLQ score. Thematic analysis indicated that PIF-oriented PBL helped students recognize realistic difficulties in clinical practice with tense emotions, and articulate their professional images and values. However, encounters in extracurricular clinical settings had diverse and meaningful impacts on PIF.

In conclusion, the limited effect of PIF-oriented PBL on PIF and SRL in this study indicated challenges in SRL-oriented education for pre-clinical year students within a teacher-centered curriculum.

\section{Chapter 6}

This chapter integrates the results of the research in Chapters 2-5, responds to the three research questions, and presents guidelines for medical education by weaving together the findings of relevant prior research.

For the first question - what contextual attributes could keep learners in a teacher-centered context from becoming self-regulated learners? - studies in Chapters 2 and 3 directly contrasted 
the effects of each learning context on learning activities. The evidence is particularly suggestive in the sense that it comes from East Asia, a region where teacher-centered curricula have strong roots. The results were successfully articulated by contrasting the data in two learning contexts and analyzing the impact on learning activities based on the three components of SRL. In summary, individual students in the teacher-centered curriculum seemed to motivate themselves to remain part of the participating groups in the curriculum (e.g., classmates, practicum groups). They reflected on themselves in contrast to the standards set by the teacher, and accordingly engaged in monotonous learning behaviors tailored to their "less failing” surroundings. A monotonous learning strategy prevailed, i.e., learning that is in line with teacher standards and group discipline. It was primarily aimed at a minimum of not failing, not straying from the group, and remembering the teacher's preferred study tasks as they were taught with the teacher-designed examinations in mind. This learning strategy is closest to "rehearsal" in SRL theory, and is often derided by the term "memorization" because it is unlike knowledge-building learning strategies such as elaboration and organization. It is consistent with the description of the learning characteristics of East Asian students as "memorization".

For the second question - what contextual attributes could be triggers for learners in a teachercentered context to become self-regulated learners?' - studies described in Chapters 2 and 3 indicated that PIF is a trigger. The study in Chapter 2, which analyzed SRL in the context of rural clinical practice, indicated that awareness of their unique identity leads them to view learning tasks as high-stakes, and to initiate learning strategies in a self-regulated manner. The study in Chapter 3, which analyzed participants of a student-designed individualized learning course, illuminated that losing the teacher-set yardstick and constructing their future selfimage as an alternative yardstick was a trigger to changes in learning behaviors. The students began to compare gaps between their present status and future self-image by self-reflection. To fill these gaps, they actively employed learning strategies used by doctors or mentors, leading to diversification of their learning strategies. When integrating the results of the two research settings in Chapters 2 and 3, we came to the conclusion that SRL can be fostered even in teacher-centered educational cultures. Also, we propose a theory that SRL-oriented education needs to i) have individuals develop their own unique future professional identity, ii) have them compare their future and current selves and relate the gap-filling objectives to the learning tasks in front of them, and iii) present a variety of learning strategies from role models that are aligned with the learning tasks.

By referring to the medical education terminology 'professional identity formation' or 'PIF', defined as the formation of self-identity with internalized values and norms of professionalism, we have developed a theory, based on the three characteristics for SRL-oriented education in the teacher-centered context, as PIF-based SRL theory.

For the third question - can we stimulate SRL when introducing education with SRL-triggering attributes? - we attempted to integrate the results of the studies described in Chapters 4 and 5 , 
which were both empirical studies of educational formats based on PIF-based SRL theory. The results in Chapters 4 and 5 differed strongly, as the former used rCBCC medical students aiming to become rural physicians while the latter was conducted in preclinical year students participating in a hybrid PBL. In a setting where medical students who aspire to become rural physicians go to their future practice site and learn under their role models, the format of PIForiented SRL theory can be useful to verbalize the value of their professionalism, self-reflect through a vision of the future, and absorb learning strategies from role models. In this context, intervention using a format based on PIF-based SRL theory promoted intrinsic motivation and strategic improvement with elaboration and critical thinking. On the other hand, in a setting of hybrid PBL within a teacher-centered curriculum, the format of PIF-oriented SRL theory cannot work efficiently to promote PIF or SRL, possibly because the overwhelming allotment of teacher-centered learning. Rather than opportunities to improve their PIF and SRL in the formal curriculum, they were improving their PIF through real-life experiences through future clinical site visits during long vacations. These findings indicate that, when we answer the third question, we would say 'Yes, we can stimulate learners' SRL when introducing education with SRL-triggering attributes or PIF-based SRL theory'. However, as a condition for this answer, it should be added that 'learning tasks based on PIF-based SRL theory must not be given in classroom settings in which PIF hardly improves'.

On consolidation of the ideas above, we emphasize that the development of SRL through PIF can be achieved by drastically reducing the number of lectures and increasing the number of classes in clinical settings, even in areas that are believed to have teacher-centered cultures. In addition, unless PIF-oriented practice is carried out at the clinical site, it will be very difficult to escape from teacher-dependent learning in the current preclinical year curriculum. This may be because students were fully immersed in teacher-instructed reproductive learning until they entered university, and it will be extremely difficult to foster SRL without them developing the self-image of a medical professional-to-be.

To address this issue, the strategy for future curriculum reform will have to be cautious yet bold. By cautious, we mean that the curriculum of Western cultures should be used as a reference, but that superficial and partial copying of a curriculum without cautious consideration should be avoided in order not to repeat the failure of the introduction of PBL. Rather, an integrative, longitudinal, workplace-based, early clinical experience program in the West can be a model for a curriculum that is equipped with a conducive environment for PIF. We emphasize that this should not be part of the curriculum, but should be introduced into the curriculum over time, similarly to or ideally more strongly than in the West to encourage a bold move away from an environment of teacher-centered learning.

As the study in Chapter 3 shows, we will be able to implement a course in which students plan their learning in a student-centered way for several months away from teacher-centered lecture classes. The FCSD at JMU might be a good platform to provide opportunities for fostering PIF-based SRL. Another option can be found in Chapter 5, in which preclinical 
students were active over the summer holidays, going to meet their role models and visit the medical sites where they would be working in the future. Fostering PIF by having students visit the medical sites where they will work in the future regularly within the preclinical curriculum therefore appears effective. In particular, it would be effective to create a class where students call on graduates working as doctors in medical practice. 


\section{Samenvatting}




\section{Hoofdstuk 1}

Klinische kennis is in snelle ontwikkeling. Medische professionals moeten hun kennis gedurende hun gehele professionele loopbaan zelfstandig bijwerken. Daarom is zelfregulatie bij leren een belangrijke competentie geworden voor medische professionals gedurende hun hele leven. Tot op heden hebben wetenschappers zelfregulatie bij leren grondig onderzocht onder de term "zelfregulerend leren" (ZRL), een begrip dat wordt gedefinieerd door drie aspecten, namelijk als "het actief deelnemen aan het eigen leerproces door studenten vanuit een metacognitief, motivatie- en gedragsperspectief".

Een aantal onderzoeken heeft benadrukt dat de leercontext bepaalt of studenten ZRL al of niet toepassen. De meeste van deze onderzoeken werden echter uitgevoerd in westerse culturele contexten. Als onderzoekers van onderwijs het belang van omgevingsaspecten bij ZRL wereldwijd benadrukken, zou er tevens aandacht besteed moeten worden aan de culturele context. Het onderwijs in Oost-Azië wordt vaak beschreven als onderwijs met een confucianistisch erfgoed dat gekenmerkt wordt door het leggen van een te sterke nadruk door familieleden, docenten en de samenleving op het behalen van examens, hogere cijfers en een hogere positie in de rangorde om toekomstig succes te garanderen. Het medisch onderwijs in Oost-Azië staat eveneens onder invloed van dit confucianistische erfgoed. Naar verluidt leren studenten uit Oost-Azië het liefst door "onderwezen te worden en te ontvangen". Docentgericht leren maakt een belangrijk deel uit van het curriculum dat in het teken staat van docentbegeleiding en -beoordeling, en dit terwijl studentgericht leren internationaal wordt aangemoedigd.

De achterblijvende ontwikkeling van ZRL bij Geneeskundestudenten vormt een probleem, daar de vervolgopleiding aiossen niet kan voorbereiden op elke uitdaging die zij na het behalen van hun diploma het hoofd moeten bieden. Het doel van dit onderzoeksproject was dan ook om te achterhalen wat de onderliggende problemen zijn. Namelijk: waardoor blijft de ontwikkeling van ZRL achter, en hoe kan het medisch onderwijs deze achterstand helpen verbeteren? We verrichtten vier afzonderlijke onderzoeken die aansloten bij de volgende drie onderzoeksvragen:

1. Welke omgevingsaspecten zouden studenten in een docentgerichte context ervan kunnen weerhouden om zelfregulerende studenten te worden?

2. Welke omgevingsaspecten zouden studenten in een docentgerichte context ertoe kunnen aanzetten om zelfregulerende studenten te worden?

3. Kunnen we ZRL stimuleren door ZRL-bevorderende aspecten in het onderwijs te introduceren? 


\section{Hoofdstuk 2}

Het in Hoofdstuk 2 beschreven onderzoek poogde de eerste en tweede onderzoeksvraag te beantwoorden door het verschil in zelfstudie te onderzoeken tussen twee duidelijk verschillende leercontexten in een docentgerichte cultuur, namelijk in Japan. We onderscheidden een groep zelfregulerende studenten in een unieke postacademische context (een medische vervolgopleiding op het platteland) die ZRL toepasten ondanks het feit dat zij docentgericht onderwijs hadden genoten tijdens hun opleiding tot basisarts in Japan. Door de basisopleidingscontext te vergelijken met de plattelandscontext tijdens hun dagelijkse zelfstudie, probeerden we in kaart te brengen welke omgevingsaspecten ZRL bevorderen of belemmeren.

We hielden interviews en verzamelden dagboekdata onder plattelandsartsen $(n=10)$ en Geneeskundestudenten $(n=11)$ in Japan die zelfstudie deden naar onbekende ziekten. Aan de hand van constructivistische, gefundeerde theorie analyseerden we drie domeinen van Zimmerman's definitie van ZRL: leergedrag, motivatie en metacognitie.

Als gevolg hiervan ontdekten we dat plattelandsartsen hun identiteit als uniek beschouwden, en dat zij zichzelf zagen als professionals die door het doen van zelfstudie een centrale rol vervulden bij het behandelen van ziekten in de plaatselijke gemeenschap. Tegelijkertijd erkenden zij dat zij het risico liepen ongepaste hulp te verlenen als hun zelfstudie ontoereikend was. Om deze high-stakes taak (een taak waarbij er veel op het spel staat) het hoofd te kunnen bieden, ontwikkelden zij diverse leerstrategieën. Geneeskundestudenten waren bang om achterop te raken en gaven er de voorkeur aan om tot de meute van medestudenten uit hetzelfde schooljaar te blijven horen. Zodoende namen zij de zelfstudie-methoden van andere studenten over en gebruikten zij monotone en homogene strategieën.

Samenvattend constateerden we dat verschillende leercontexten Oost-Aziatische studenten er niet van weerhouden om zelfregulerend te zijn. Het zich bewust zijn van hun unieke identiteit zorgt ervoor dat zij leertaken als high-stakes beschouwen en dat zij leerstrategieën op zelfregulerende wijze aanwenden. Aan de andere kant zorgen docentgerichte onderwijssystemen ervoor dat studenten zichzelf zien als "behorend tot de meute" en dat zij taken als low-stakes beschouwen, waardoor zij zich bedienen van strategieën die niet zelfregulerend zijn.

\section{Hoofdstuk 3}

Het onderzoek in Hoofdstuk 2 gaf aan dat een docentgerichte context Geneeskundestudenten kon beletten ZRL toe te passen, terwijl een studentgerichte context het gebruik juist kon bevorderen. Onderzoekers hebben de ontwikkeling van ZRL in een docentgerichte context echter nog niet rechtstreeks vergeleken met diezelfde ontwikkeling in een studentgerichte context in de opleiding tot basisarts. Daarbij blijft het onduidelijk hoe een verandering in de context naar studentgericht leren het gebruik van ZRL beïnvloedt bij studenten die niet anders gewend zijn dan docentgericht leren. 
We hielden drie focusgroepen met 13 Japanse Geneeskundestudenten die van een traditioneel curriculum dat uit didactische hoorcolleges en veel summatieve toetsen bestond, een keuzeblok van zeven maanden startten. In dit vrije blok voor coassistenten of FCSD (Free Course Student Doctor) wordt de nadruk gelegd op een door de student ontworpen individueel leerplan, waarbij de student ondersteuning en formatieve feedback ontvangt van zelf uitgekozen mentoren. We hielden ook twee focusgroepen met zeven studenten die gedurende dezelfde periode in het docentgerichte curriculum waren gebleven. We vroegen studenten om hun 1) motivatie, 2) leerstrategieën, en 3) zelfreflectie op zelfstudie voor en tijdens de genoemde periode te bespreken. De data werden geanalyseerd aan de hand van een thematische analyse en code-vergelijking tussen de twee cohorten.

De resultaten lieten zien dat de niet-FCSD-participanten hun motivatiestatus omschreven als behorend tot de meute die zich liet bepalen door de maatstaf van de docent. Tijdens het reflecteren richtten zij zich op het minimaliseren van de kloof tussen henzelf en de maatstaf van hun docent door zich te bedienen van strategieën die als monotoon en homogeen worden beschouwd (bijv. van buiten leren). FCSD-participanten beschreven dat zij de maatstaf van hun docent loslieten en als alternatieve maatstaf hun eigen toekomstig zelfbeeld construeerden. Door middel van zelfreflectie onderzochten ze of er lacunes bestonden tussen hun huidige status en hun toekomstig zelfbeeld. Om deze lacunes op te vullen bedienden ze zich actief van leerstrategieën die door artsen en mentoren werden gebruikt, wat leidde tot diversificatie van hun leerstrategieën.

Samenvattend kunnen we stellen dat een verandering in de context naar studentgericht leren ZRL kan bevorderen, zelfs bij studenten die niet anders gewend zijn dan docentgericht leren. In de studentgerichte context begonnen studenten hun professionele identiteit te formuleren, op hun leergedrag te reflecteren en naar verschillende leerstrategieën te zoeken door modellen van hun toekomstige "zelf" aan te roepen.

\section{Hoofdstuk 4}

In de onderzoeken in Hoofdstuk 2 en 3 werd geconstateerd dat een verandering in de context naar een context waarin studenten de mogelijkheid krijgen een professionele identiteit te formuleren, van belang kan zijn voor de verbetering van ZRL. Gezien de populariteit en toepasbaarheid van de term "professionele-identiteitsvorming" (PIF") in het medisch onderwijs, gebruikten we deze term om het omgevingsaspect te beschrijven. In de onderzoeken in Hoofdstuk 2 en 3 kon echter geen oorzakelijk verband worden aangetoond tussen een PIFgerichte context en de verbetering van ZRL. Daarom ontwikkelden we op basis van de in Hoofdstuk 2 en 3 getoonde onderzoeksbevindingen een PIF-gerichte onderwijsvorm en onderzochten we de effecten hiervan op ZRL bij studenten in de klinische fase.

$1 \quad$ PIF $=$ Professional Identity Formation. 
Hoofdstuk 4 beschrijft een multimethodisch onderzoek dat de effecten onderzocht van een PIF-gerichte onderwijsvorm dat ontworpen was om Geneeskundestudenten te helpen om tijdens een klinische coschap: 1) een toekomstig beeld van "zichzelf" als professional te formuleren, en 2) diepgaande gesprekken over medisch professioneel handelen te voeren met rolmodellen. De onderwijssetting was een plattelandsgemeenschapsgerichte klinische coschap, in het navolgende rCBCC (rural community-based clinical clerkship) te noemen, voor studenten aan de medische universiteit in Jichi, Japan (Jichi Medical University; JMU). Dit coschap bood masterstudenten de mogelijkheid om werkervaring op te doen op hun toekomstige werkplek onder het directe toezicht van aiossen (de rolmodellen). Aan dit PIF-gerichte rCBCC namen 41 vijfdejaars studenten van JMU deel, terwijl een controlegroep van 41 vijfdejaarsstudenten de oorspronkelijke onderwijsvorm van het rCBCC onderging. We vergeleken de veranderingen in ZRL tussen de twee groepen met behulp van 15 categorieën van de vragenlijst over gemotiveerde leerstrategieën (de Motivated Strategies for Learning Questionnaire; MSLQ). Daarbij onderzochten we aan de hand van een thematische analyse van de data uit de vragenlijsten en interviews hoe het PIFgerichte rCBCC hun ZRL veranderde.

Op basis van deze analyses constateerden we dat in de PIF-gerichte rCBCC-groep zowel de intrinsieke doelgerichtheid $\left(p=0,005, \varepsilon^{2}=0,096\right)$ als het kritisch denken $\left(p=0,041, \varepsilon^{2}=0,051\right)$ licht verbeterde. Uit de kwalitatieve analyse kwam naar voren dat zij "professionele verantwoordelijkheid" als een drijfveer voor zelfstudie begonnen te zien. Het zich bewust worden van hun "verantwoordelijkheid" zorgde ervoor dat zij echtheid en toepasselijkheid van medische kennis nastreefden en maakte dat zij kritisch gingen nadenken over de leerstof. Daarbij begonnen zij de verwerking van leerstof af te stemmen op taakprocessen uit de praktijk.

Het onderzoek in Hoofdstuk 4 toonde aan dat PIF-gericht onderwijs enkele effecten heeft op de verbetering van ZRL. Het bevorderen van PIF kan belangrijk zijn voor het stimuleren van intrinsiek ZRL-gedrag door studenten.

\section{Hoofdstuk 5}

Hoofdstuk 5 beschrijft een multimethodisch onderzoek dat de effecten onderzocht van een PIFgerichte onderwijsvorm bestemd voor een preklinisch, hybride PGO-curriculum in een docentgerichte cultuur. We ontwikkelden deze onderwijsvorm om studenten uit de preklinische fase te helpen om vóór elke PGO-sessie: 1) hun beeld van hun toekomstige "zelf" als professional te formuleren, en 2) diepgaande gesprekken over medisch professioneel handelen te voeren met rolmodellen.

In het kwantitatieve deel werd een gerandomiseerd onderzoek met controlegroep en crossover-design verricht waaraan 112 studenten van JMU uit de preklinische fase deelnamen. Gedurende zes PGO-sessies die elk een dag duurden, ervoeren groep A ( $n=56 ; 18$ vrouwen; 38 mannen; gemiddelde leeftijd: \pm SD 21,5 $\pm 0,7)$ en $\mathrm{B}(n=56$; 11 vrouwen; 45 mannen; gemiddelde 
leeftijd: \pm SD 21,7 $\pm 1,0)$ PIF-gericht pre-PGO-onderwijs: drie sessies in de eerste en drie sessies in de tweede helft van het jaar. Met behulp van respectievelijk de professionele-identiteitstest (Professional Identity Essay; PIE) en de vragenlijst over gemotiveerde leerstrategieën (MSLQ) werden de PIF- en ZRL-niveaus tussen de twee groepen met elkaar vergeleken. In het kwalitatieve deel werd aan de hand van de PIE-beschrijving een thematische analyse uitgevoerd van de factoren die gedurende de onderzoeksperiode een verbetering in PIF teweegbrachten.

Een two-level regressieanalyse liet een geleidelijke, lichte verbetering in PIF-stadia zien $\left(R^{2}=\right.$ $0,069)$, ongeacht de interventie. PIF-gericht pre-PGO-onderwijs op zichzelf zorgde niet voor een significante verbetering van PIE-stadium of MSLQ-score. Uit een thematische analyse bleek dat PIF-gericht PGO studenten hielp om realistische moeilijkheden in de klinische praktijk die voor gespannen emoties zorgden te herkennen en om hun professionele beeld en waarden te formuleren. Ervaringen in klinische settings buiten het curriculum om hadden daarentegen diverse en zinvolle gevolgen voor PIF. De conclusie was dat het geringe effect van PIF-gericht PGO op PIF en ZRL in deze studie erop duidde dat er binnen het ZRL-onderwijs voor studenten in de preklinische fase binnen een docentgericht curriculum nog hordes te nemen zijn.

\section{Hoofdstuk 6}

In dit hoofdstuk worden de resultaten van het onderzoek uit Hoofdstuk $2 \mathrm{t} / \mathrm{m} 5$ gebundeld, de drie onderzoeksvragen beantwoord en richtlijnen voor medisch onderwijs gegeven door de bevindingen van relevante eerdere onderzoeken met elkaar te verweven.

Wat betreft de eerste vraag (Welke omgevingsaspecten zouden studenten in een docentgerichte context ervan kunnen weerhouden om zelfregulerende studenten te worden?), vergeleken de onderzoeken uit Hoofdstuk 2 en 3 de effecten die elke leercontext had op de leeractiviteiten rechtstreeks met elkaar. Het bewijs is vooral veelzeggend omdat het afkomstig is uit Oost-Azië, een gebied waar docentgerichte curricula stevig verankerd zijn. De resultaten werden met goed gevolg verkregen door de gegevens uit twee leercontexten met elkaar te vergelijken en aan de hand van de drie aspecten van ZRL de invloed op leeractiviteiten te analyseren. Samenvattend leken de afzonderlijke studenten uit het docentgerichte curriculum zichzelf te motiveren om tot de aan het curriculum deelnemende groepen te blijven behoren (zoals klasgenoten, practicumgroepen). Tijdens zelfreflectie vergeleken zij zichzelf aan de hand van de maatstaf van de docent en zodoende vertoonden zij monotoon leergedrag dat afgestemd was op hun "minder falende" omgeving. Er heerste een monotone leerstrategie, dat wil zeggen dat het leren was afgestemd op de maatstaf van de docent en op de groepsdiscipline. Het voornaamste doel van leren was het behalen van toetsen met de minst mogelijke inspanning, het niet te ver achter op de groep geraken, en het onthouden van de favoriete studietaken van de docent, aangezien deze werden aangeleerd om de door de docent ontwikkelde toetsen te kunnen maken. Deze leerstrategie komt het meest overeen met "herhaling" uit de ZRL-theorie en wordt vaak weggezet 
als "van buiten leren" omdat het anders is dan leerstrategieën waarbij sprake is van kennisopbouw, zoals verdiepen en organiseren. Daarbij sluit het aan op de typering van het leergedrag van OostAziatische studenten als "van buiten leren".

Wat betreft de tweede vraag (Welke omgevingsaspecten zouden studenten in een docentgerichte context ertoe kunnen aanzetten om zelfregulerende studenten te worden?), gaven de in Hoofdstuk 2 en 3 beschreven onderzoeken aan dat PIF een prikkel is. Het onderzoek in Hoofdstuk 2, waarin ZRL werd onderzocht in de klinische praktijk op het platteland, gaf aan dat het zich bewust zijn van hun unieke identiteit ervoor zorgde dat zij leertaken als high-stakes beschouwden en dat zij leerstrategieën op zelfregulerende wijze aanwendden. Het onderzoek in Hoofdstuk 3, dat participanten van een blok met individueel leren op basis van een zelf ontworpen leerplan onderzocht, bracht aan het licht dat het loslaten van de maatstaf van de docent en het construeren van een eigen toekomstig zelfbeeld als alternatieve maatstaf een prikkel was voor veranderingen in leergedrag. Door middel van zelfreflectie begonnen studenten lacunes tussen hun huidige status en hun toekomstig zelfbeeld te vergelijken. Om deze lacunes op te vullen bedienden ze zich actief van leerstrategieën die door artsen en mentoren werden gebruikt, wat leidde tot diversificatie van hun leerstrategieën. Bij het bundelen van de resultaten van de twee onderzoekssettings uit Hoofdstuk 2 en 3 kwamen we tot de conclusie dat ZRL zelfs in culturen met docentgericht onderwijs kan worden bevorderd. We stellen ook de theorie voor dat ZRLonderwijs erop gericht moet zijn om: i) studenten hun eigen unieke toekomstige professionele identiteit te laten ontwikkelen, ii) studenten hun toekomstige en huidige zelf te laten vergelijken en hun doelen om deze lacunes op te vullen te laten verbinden aan de leertaken die zij voor zich hebben, en iii) studenten kennis te laten maken met een verscheidenheid aan leerstrategieën van rolmodellen die passend zijn voor de leertaken.

Met behulp van de medisch onderwijskundige term "professionele-identiteitsvorming" of "PIF", dat verwijst naar de vorming van een eigen identiteit waarbij professionaliteitsnormen en -waarden zijn eigen gemaakt, hebben we aan de hand van de drie kenmerken van ZRL-onderwijs in de docentgerichte context een theorie ontwikkeld, te weten PIF-gericht ZRL-theorie.

Wat betreft de derde vraag (Kunnen we ZRL stimuleren door ZRL-bevorderende aspecten in het onderwijs te introduceren?), hebben we geprobeerd de resultaten van de onderzoeken uit Hoofdstuk 4 en 5, beide empirische studies van onderwijsvormen die uitgingen van PIF-gericht ZRL-theorie, te synthetiseren. De resultaten van Hoofdstuk 4 en 5 verschilden sterk, omdat het eerste onderzoek was verricht met rCBCC-Geneeskundestudenten die plattelandsarts wilden worden terwijl het laatste onderzoek werd gehouden onder studenten uit de preklinische fase die deelnamen aan een hybride PGO-vorm. In een setting waar Geneeskundestudenten die ernaar streven om plattelandsarts te worden hun toekomstige praktijk bezoeken en aldaar onder begeleiding van hun rolmodellen leren, kan een onderwijsvorm die uitgaat van PIF-gericht ZRLtheorie zinvol zijn om de waarde van hun professionaliteit te formuleren, op zichzelf te reflecteren aan de hand van een toekomstbeeld, en om leerstrategieën van rolmodellen te absorberen. In deze context leidde de interventie waarvoor een onderwijsvorm werd gebruikt die uitging van 
PIF-gericht ZRL-theorie tot meer intrinsieke motivatie en een verbetering van leerstrategieën met meer verdieping en kritisch denken. In een setting waar sprake is van een hybride PGO-vorm binnen een docentgericht curriculum daarentegen, kan de onderwijsvorm die uitgaat van PIFgericht ZRL-theorie niet effectief bijdragen aan PIF of ZRL, mogelijk vanwege het grote overwicht dat docentgericht leren heeft. In plaats dat studenten hun PIF en ZRL verbeterden door kansen in het formele curriculum aan te grijpen, verbeterden zij hun PIF door tijdens lange vakanties toekomstige klinische praktijken te bezoeken en zodoende praktijkervaring op te doen. Deze bevindingen maken aannemelijk dat het antwoord op de derde vraag zou moeten luiden "Ja, we kunnen het ZRL van studenten stimuleren als we ZRL-bevorderende aspecten of PIF-gericht ZRL-theorie in het onderwijs introduceren”. Hierbij moet echter een kanttekening worden geplaatst door de toevoeging: "leertaken die uitgaan van PIF-gericht ZRL-theorie moeten niet worden aangereikt in klassikale settings waar PIF nauwelijks verbetert".

Ter bekrachtiging van bovenstaande ideeën, benadrukken we dat de ontwikkeling van ZRL door middel van PIF gerealiseerd kan worden door het aantal hoorcolleges drastisch terug te brengen en het aantal lessen in klinische settings te vermeerderen, zelfs in gebieden waarvan wordt aangenomen dat er een docentgerichte cultuur is. Bovendien zal het erg moeilijk zijn om in het huidige preklinische curriculum te ontsnappen aan docentgericht leren, tenzij de PIFgerichte onderwijsactiviteiten in de klinische praktijk worden uitgevoerd. Dit kan komen doordat studenten tot het moment dat zij naar de universiteit gingen volledig ondergedompeld waren geweest in reproducerend leren dat door de docent werd aangestuurd. Het zal dan ook buitengewoon moeilijk zijn om ZRL te stimuleren wanneer zij zich geen zelfbeeld van een toekomstig medisch professional hebben gevormd.

Om dit probleem aan te pakken, zal het beleid ten aanzien van toekomstige curriculumherziening voorzichtig maar gewaagd moeten zijn. Met voorzichtig bedoelen we dat het curriculum van westerse culturen gebruikt moet worden als richtsnoer, maar dat het oppervlakkig en gedeeltelijk overnemen van een curriculum zonder daar eerst voorzichtig over na te denken vermeden moet worden om te voorkomen dat hetzelfde lot wordt ondergaan als bij de invoerring van PGO. In plaats daarvan kan een westerse integrale en longitudinale opleiding waarbij werkplekleren en klinische ervaring in de bachelorfase centraal staan als voorbeeld dienen voor een curriculum dat is voorzien van een PIF-bevorderende omgeving. We benadrukken dat deze omgeving geen onderdeel van het curriculum moet zijn, maar dat deze stapsgewijs in het curriculum moet worden geïntroduceerd, op dezelfde manier als in het Westen of liefst nog krachtiger om een gewaagde stap uit een docentgerichte leeromgeving te stimuleren.

Zoals het onderzoek in Hoofdstuk 3 aantoont, zijn we in staat om een blok te introduceren waarbij docentgerichte hoorcolleges plaats hebben gemaakt voor de gelegenheid om gedurende enkele maanden op studentgerichte wijze een eigen leertraject uit te stippelen. Het FCSD aan de JMU zou een goed platform kunnen zijn voor het bieden van kansen om PIF-gericht ZRL te stimuleren. Een andere mogelijkheid vindt men in Hoofdstuk 5, waar preklinische studenten tijdens hun zomervakantie actief waren door hun rolmodellen te ontmoeten en de klinische 
praktijken te bezoeken waar zij in de toekomst zouden komen te werken. Het is daarom aannemelijk dat PIF effectief kan worden bevorderd door studenten in de preklinische fase regelmatig de medische praktijken waar zij in de toekomst zullen werken te laten bezoeken. Het zou met name effectief zijn om een les te organiseren waarbij studenten de gelegenheid hebben om met aiossen in gesprek te gaan. 

Valorization 


\section{Relevance}

The results of this research have significant social and economic implications. Although the internationalization of medical education is progressing, there are still some areas of medical schools that emphasize teacher-centered learning, with lecture-type classes and teacher-led practical training taking center stage. Until now, the evidence for medical education has originated mainly in the West, and the shift to student-centered learning in medical education has been promoted mainly in the West. East Asia, including Japan, where this study was conducted, have attempted to address the shift to student-centered learning by "transplanting" learning methods, and have thus dismissed institutions' "rejection" of it as due to cultural differences alone. Of course, the cost paid simply for the "transplantation" of new learning methods is enormous.

In this study, we first examined how teacher-centered learning actually affects students' learning behavior in Chapters 2 and 3 in an area in which teacher-centered learning has traditionally been dominant. Results showed that the elements included in a teacher-centered learning context make students passive learners, but that they become active self-regulating learners if they leave the teacher-centered curriculum and are placed in a different learning context. Being able to verbalize a learning context in which students currently engaged in teacher-centered learning can in future become self-regulated learners without having to leave their cultural sphere will lead to more effective curriculum development, and the social significance of this study will become clear. In addition, Chapters 4 and 5 examined the effectiveness of educational interventions formatted on contextual attributes promoting selfregulated learning (SRL). We believe that this represents a shift from theory to practice and offers a framework for new curriculum development in regions traditionally considered to be teachercentered learning cultures. Given the cost-effectiveness of curriculum development from evidence within a culture compared to the cost-effectiveness of partial "transplantation" of educational methods across cultures, this study might also be economically meaningful.

\section{Target groups}

The ultimate target of this study's results is the patient. SRL, the basis of the study theme, is the cornerstone of lifelong professional development. More than anyone else, it is in the best interest of the patient that physicians update their skills and techniques in a self-regulating manner without neglecting to keep up with advances in medical education. A second underlying theme, improving professional identity formation (PIF), also benefits patients. The general public reading this paper may have been surprised to learn that in a teacher-centered learning culture, learners who enter medical school after a fierce examination war lacked a vision of their future in becoming a doctor. Nevertheless, this is the reality of the teacher-centered learning culture, at 
least in medical schools in Japan, and if this study, which shows that improving PIF is the trigger for the aforementioned lifelong enhancement power, is the driving force behind a shift in university curricula, then the ultimate target is indeed the patient.

The next target audience for this study is medical school faculty. In particular, these are teachers in a teacher-centered curriculum. It is difficult for teachers in a teacher-centered educational culture to understand the trends in medical education around the world as they practice education as part of their educational culture. In particular, the current state of evidence for medical education, emanating mainly from the West, had stopped the discussion with the simplistic response that student-centered learning was "culturally different from our own." Against this, we hope that the research in Chapters 2 to 5 helps them realize that the education they take for granted is turning students into passive learners. The message is that they need to move away from their preconceived notions of culture. The results of Chapter 5 show that the students are more active than they thought. In other words, in order to shape their own future vision, students tried to visit medical sites where they might work in the future and meet senior doctors during long vacations outside of the curriculum. These results show that the proper role of teachers in teacher-centered learning is indeed nothing short of bold reform of the curriculum.

\section{Activities/Products}

Based on the PIF-based SRL theory established based on Chapters 2 and 3, Chapters 4 and 5 developed forms of educational interventions. The studies in these chapters tested these interventions in the actual curriculum. Although there is much room for improvement and further multi-center validation is needed, an online dialogue platform for educational interventions in clinical practice (Chapter 4) and pre-clinical PBL (Chapter 5), as well as manuals for instructors conducting the educational interventions, are available from the author by request.

\section{Innovation}

The results of this study are innovative because few studies have examined in detail why passive learning behaviors occur in teacher-centered educational cultures. Chapters 2 and 3 offer one explanation for the mechanism. Second, the study directly tested the causal relationship between the concept of PIF and SRL theory; the results of Chapters 2 and 3 offered the hypothesis that improved PIF leads to improved SRL, and the intervention studies in Chapters 4 and 5 tested this hypothesis deductively. Although not all of the hypotheses were correct, a certain cause-andeffect relationship could be explained. Thus, this is an innovative study in the sense that it combines two concepts that until now have been difficult to discuss directly in medical education. 


\section{Schedule \& Implementation}

The format of the educational intervention based on the PIF-based SRL theory shown in Chapters 4 and 5 is currently being improved for the next verification studies. Regarding the rural community-based clinical clerkship at JMU in Chapter 4, we are currently improving e-learning contents for faculty development guidance by enriching the manual to support the conduct of PIF-oriented educational interventions. For the PIF-oriented educational intervention for hybrid PBL conducted in Chapter 5, we are planning to start an empirical study next year after improving not only the method of PIF development but also various factors such as the scenario used for PBL, and the environment, frequency, and schedule for PBL learning. In addition, none of the studies has been validated at multiple sites. We have now obtained consent to cooperate with two institutions in Japan and plan to introduce an educational intervention based on PIF-based SRL theory in these institutions in two years.

Although it will take several years, we would like to work on reforming the overall curriculum based on the PIF-based SRL theory, albeit in a teacher-centered learning culture. 
Acknowledgements 
I would like to express my deep gratitude to all those who have helped me in my long journey from the start of my Master of Health Professional Education (MHPE) degree to the end of my $\mathrm{PhD}$ course at Maastricht University.

First, I want to show my gratitude for the education I received in MHPE. In particular, I feel most grateful to the main supervisor, the late Arno Muijtjens, for his way of supervision. It has become the basis of the ideas of this thesis. His supervision did not involve spoon-feeding, which I had experienced in my school days in Japan, but rather gave constructive feedback only when I spontaneously asked questions verbally. The feedback was not conclusive, but rather questioning comments that encouraged self-reflection. I am deeply saddened by his passing. I would also like to thank Dr. Joost Dijkstra, the second supervisor. My second year at MHPE was so challenging, but his charming humanity and supportive behavior was a really moral support.

Next, I would like to thank Dr. Renée Stalmeijer, who further refined my MHPE dissertation and helped me publish it in BMC Medical Education as a medical education research paper, for the first time in my life. As previously a positivist, I had found it difficult to conduct qualitative analysis from a constructivist perspective. It was her advice that led me to that deep understanding. Prof. Reiko Murakami, Prof. Shizukiyo Ishikawa, and Prof. Hitoaki Okazaki at Jichi Medical University, and Dr. Makoto Kikukawa at Kyushu University were also great supervisors for its publication. It will be a lifelong asset to be an author with them.

During the four-year journey of the PhD program, the cooperation of many co-authors was essential. I would like to thank Prof. Motoyuki Nakaya at Nagoya University and Prof. Hitoaki Okazaki at Jichi Medical University for the time and effort in the qualitative data analysis. My conversations with them have broadened my medical educator's values and perspectives. I would like to thank Dr. Teppei Sasahara, Dr. Yu Yamamoto, Prof. Akira Gomi, and Prof. Masami Matsumura at Jichi Medical University for their understanding of my research protocol. I would like to thank Prof. Kazuhiko Kotani for allowing me to use the community-based clinical clerkship as a venue to validate my educational intervention. Dr. Yoshikazu Asada is a very talented medical education engineer who has been instrumental in the technical support of Google Forms and Moodle, as well as providing full cooperation in the management of quantitative data. Prof. Adam Jon Lebowitz, with his excellent linguistic skills, was very precise in proofreading my English. Ms. Akemi Watanabe, a staff member of the Medical Education Center of Jichi Medical University, quickly organized the various data in order to carry out the study. Ms. Yasuko Koguchi, Ms. Kazumi Kato, and Ms. Kaori Tasaki also supported me in my daily work. Thanks to them, I was able to immerse myself in this research.

I would like to thank my dear mentors Dr. Jimmie Leppink and Prof. Cees van der Vleuten, who have been with me on the entire journey of my $\mathrm{PhD}$. The more I argued with them, the more I was overwhelmed by the depth of their insights. They accurately pointed out the weaknesses of my papers while they encouraged me mentally. As a result, I was able to do the most difficult "homework" of my life. They spent a lot of time and effort to proofread this PhD thesis, especially this year, even when they were in lockdown due to COVID-19. I would like to thank them once 
again for their dedication and guidance as educators in the midst of a difficult social situation.

In the last paragraph of this chapter, I would like to express my deepest gratitude and gratefulness to my family members. To my wife Rie and our three children, Tomoshi, Reiji and Minori, my mother Yoshiko, and my sister Noriko and brother Hitoshi: I am proud to have you all as my family. I sincerely regret the sacrifice of so much family time to carry out the study. I was able to complete this course in four years, as originally planned, because they were patient with me and allowed me to spend time with my $\mathrm{PhD}$ project. I would also like to thank my beloved father, Takeshi, who passed away from an illness during my four-year journey. He was the one who instilled in me the patience and effort to overcome difficulties. My journey as an educational scientist will not end. This is because, in the midst of a period with unpredictable difficulties coming one after another, medical education in Japan has to go the way it should go, and become what it should be. 

Curriculum Vitae 
Yasushi Matsuyama was born in Japan in 1975. He studied medicine at Jichi Medical University (JMU) between 1995 and 2001. He received the degree of Doctor of Medicine from JMU in 2001. He attended the residency program in Shizuoka Prefectural General Hospital from 2001 to 2003. After residency training, he worked as a medical practitioner in some hospitals in Shizuoka Prefecture. In 2007, he entered the PhD course for medicine in the graduate school at JMU and studied clinical immunology and rheumatology under Professor Seiji Minota. His thesis theme was 'clinical implications of interleukin-33 in rheumatoid arthritis.' He received a $\mathrm{PhD}$ degree for medicine with the thesis of the same title. In 2011, he moved to Ito municipal hospital and started working as a general physician. His involvement with medical education began in 2012, when he was appointed as the vice leader of the clinical training center and engaged in coordination of the residency program. He also exerted himself to build up the simulation center at Ito Municipal Hospital. In 2013, he entered the Master of Health Professions Education program at Maastricht University to deepen his understandings of medical education. While studying for his Masters, he developed an interest in assessment development.

In 2014, he moved to the current institution, the Medical Education Center in JMU and was appointed Chairman of the Board of Sougou-hantei Shiken (a yearly comprehensive assessment test). That position encouraged him to explore the applicability of progress testing in East Asian culture. In 2015, he completed the Master of Health Professions Education program at Maastricht University with his master's thesis, titled 'A first trial of progress testing in East Asia: Students' perceptions of the new testing procedure.' The supervisors of the thesis were Dr. A.M.M. Muijtjens and Dr. Joost Dijkstra. His strong interest in assessment development and their excellent instruction eventually resulted in the first publication of his medical educational research, in BMC Medical Education in 2016.

He has been in his current position of Associate Professor at the Medical Education Center of Jichi Medical University since February 2018. He is now a member of the editorial board of 'Igakukyoiku,' the official journal of the Japan Society for Medical Education.

He received the Distinguished Graduate Award of Jichi Medical University in 2001, the Research Encouragement Award of Jichi Medical Graduate School in 2010, and the Kaketa Award (award for best paper by the Japan Society for Medical Education) in 2018. 


\section{School of
Health Professions
Education}

PhD dissertations completed in 2019-20 at Maastricht University

Giuliani, M. (19-05-2020) A Critical Review of Global Curriculum Development, Content and Implementation in Oncology

Schreurs, S. (20-03-2020) Selection for medical school; the quest for validity

Schumacher, D. (19-03-2020) Resident Sensitive Quality Measures: Defining the Future of Patient-Focused Assessment

Sehlbach, C. (21-02-2020) To be continued... Supporting physicians' lifelong learning

Kikukawa, M. (17-12-2019) The situated nature of validity: Exploring the cultural dependency of evaluating clinical teachers in Japan

Kelly, M. (10-12-2019) Body of knowledge. An interpretive inquiry into touch in medical education

Klein, D. (06.11.2019) The performance of medical record review as an instrument for measuring and improving patient safety

Bollen, J. (01.11.2019) Organ donation after euthanasia: medical, legal and ethical considerations

Wagner-Menghin, M. (25-09-2019) Self-regulated learning of history-taking: looking for predictive cues

Wilby, K. (02-07-2019) When numbers become words: Assessors' processing of performance data within OSCEs

Szulewski, A. (20-06-2019) Through the eyes of the physician: Expertise development in resuscitation medicine

McGill, D. (29-05-2019) Supervisor competence as an assessor of medical trainees; Evaluating the validity and quality of supervisor assessments

Van Rossum, T. (28-02-2019) Walking the tightrope of training and clinical service; The implementation of time variable medical training 